\title{
Role of Munc13 Isoforms in Regulating Large Dense Core Vesicle Exocytosis in Chromaffin Cells
}

\author{
Dissertation \\ to acquire the doctoral degree in mathematics and natural sciences \\ "Doctor rerum naturalium" \\ at the Georg-August-Universität Göttingen \\ in the doctoral degree programme Molecular Physiology of the Brain \\ at the Georg-August University School of Science (GAUSS)
}

Submitted by

Kwun Nok Mimi Man

from Hong Kong

Göttingen, 2015 


\section{Members of the Thesis Committee:}

Prof. Dr. Nils Brose (Reviewer)

Department of Molecular Neurobiology, Max Planck Institute of Experimental Medicine

Prof. Dr. Erwin Neher (Reviewer)

Emeritus Group Membrane Biophysics, Max Planck Institute for Biophysical Chemistry

Prof. Dr. Reinhard Jahn

Department of Neurobiology, Max Planck Institute for Biophysical Chemistry

\section{Additional Members of the Examination Board:}

Prof. Dr. Thomas Dresbach

Department of Anatomy and Embryology, Georg-August-Universität Göttingen; University Medical Center Göttingen

Prof. Dr. Tobias Moser

Institute for Auditory Neuroscience \& InnerEarLab, University Medical Center Göttingen

Dr. Camin Dean

European Neuroscience Institute

Date of oral examination: 30.04 .2014 . 
I hereby declare that the thesis had been written independently and with no other sources and aids than quoted. 


\section{Acknowledgements}

I would like to thank, first and foremost, my supervisor Dr. Sonja Wojcik, for her help throughout the three and a half years during my doctoral studies. She is a thoughtful and meticulous scientist and I have learnt a great deal from her. I thank her also for letting me work independently and being available for me when I have difficulties in my work. She helped me with sincerity when I face problems in my life, giving me useful opinions in many aspects. I would also like to thank Prof. Nils Brose, who provided the opportunity for me to work in his lab, for much valuable discussions on the project, as well as for providing abundant resources and a comfortable working environment in the lab.

I am grateful to Dr. Jeong-Seop Rhee, who helped me with electrophysiological work and gave much advice and ideas on how to improve the quality of my results. My thanks also go to Dr. Benjamin Cooper and Dr. Cordelia Imig, who collaborate with us in producing electronic microscopy data. I am grateful to Prof. Jakob Sørensen and Dr. Paulo Pinheiro for their help on chromaffin cell experiments. In addition, I thank Dr. Kerstin Reim for arranging animals for me so that I can work on them. I am also thankful to Dr. Sangyong Yung, who taught me how to patch at the very beginning. I thank my colleagues in the Department of Molecular Neurobiology, MPI-EM, for their help in experimental work, scientific discussions and good company.

I am indebted to my family, whose continuous support fuels me in the pursuit of a career in biological science. Finally, I would like to thank my husband for always being there for me, supporting me in many aspects and for the fun and meaning he brings into my life. 


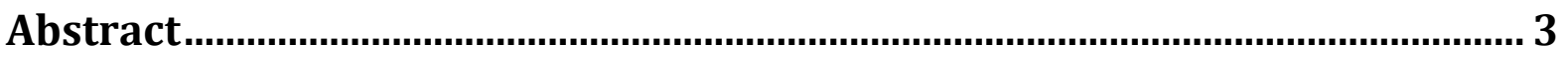

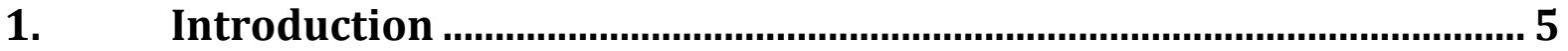

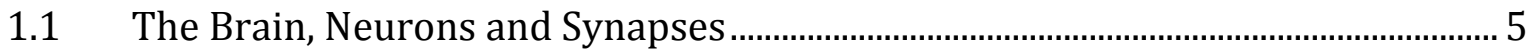

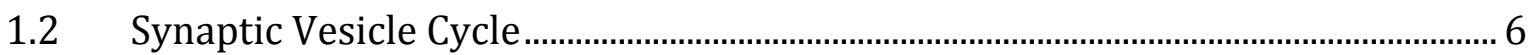

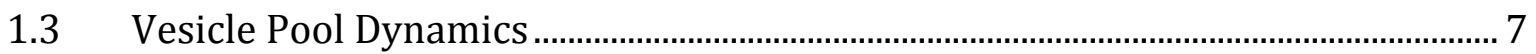

1.3.1 Vesicle Pool Dynamics in Chromaffin Cells ............................................................. 8

1.4 Comparison between Synaptic Vesicle Exocytosis and Large Dense Core Vesicle Exocytosis............................................................................................................11

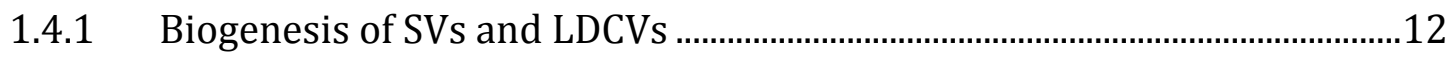

1.4.2 Docking of SVs and LDCVs...............................................................................

1.5 Mechanisms of Fast Vesicular Exocytosis .................................................................16

1.5.1 SNARE Proteins as the Core Fusion Machinery …………………......................16

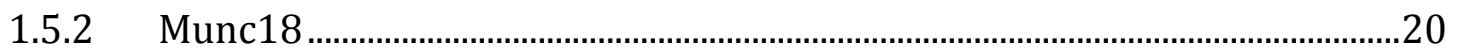

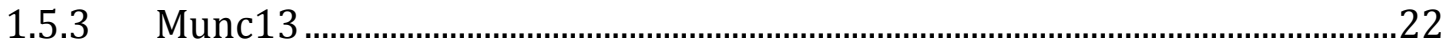

1.5.3.1 Domain Structure of Munc13s.......................................................................22

1.5.3.2 Expression Pattern of Munc13s ....................................................................24

1.5.3.3 Role of Munc13 Isoforms in Priming and Docking ...................................27

1.5.3.4 Proposed Mechanisms of Munc13 Function................................................32

1.5.3.5 Short-term Synaptic Plasticity Characteristics of Munc13 Isoforms...35

1.6 Role of CAPS in Priming and Docking ……........................................................................36

$1.7 \quad$ Scope of the Thesis.....................................................................................................

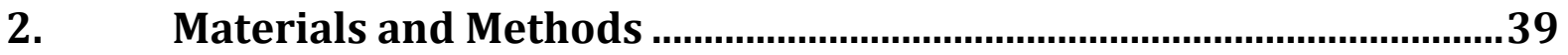

$2.1 \quad$ Transgenic Mouse Lines and Genotyping ……..............................................................39

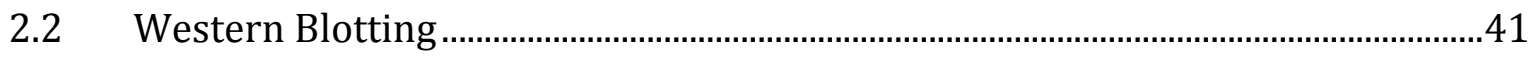

$2.3 \quad$ Chromaffin Cell Culture..................................................................................................

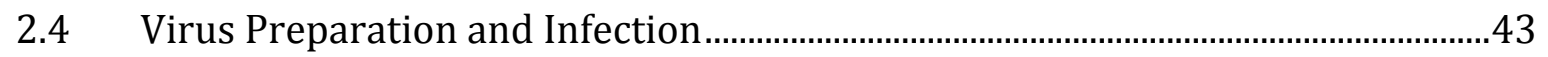

$2.5 \quad$ Electrophysiological Recordings and Data Analysis......................................................44

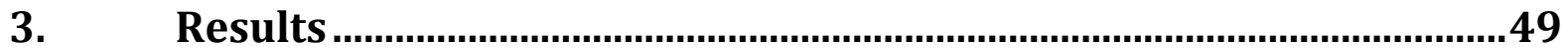

3.1 Expression of Munc13 Isoforms in the Adrenal Gland ...............................................49

3.2 LDCV Exocytosis is Intact in Munc13-1 KO Chromaffin Cells.................................53

3.3 LDCV Exocytosis is Intact in Baiap3 KO Chromaffin Cells ......................................54 
3.4 LDCV Exocytosis is Intact in Munc13-3 KO Chromaffin Cells..................................55

3.5 ubMunc13-2 is Required for LDCV Exocytosis in Chromaffin Cells and a Role for Munc13-1 Becomes Apparent in the Absence of Munc13-2 ............................57

3.6 ubMunc13-2 is Required for LDCV Release Triggered by Depolarization .........61

3.7 ubMunc13-2 and Munc13-1 Differentially Rescue LDCV Exocytosis in Munc131/2 DKO Chromaffin Cells.........................................................................................62

3.8 Baiap3 is Not Required for LDCV Exocytosis in Chromaffin Cells........................66

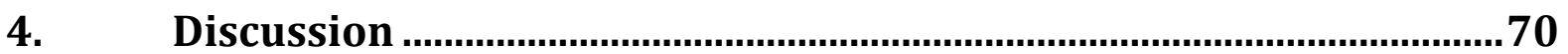

4.1 Contributions of Munc13 Isoforms to LDCV Exocytosis in Chromaffin Cells ....70

4.1.1 Contributions of Munc13-1 to LDCV Exocytosis in Chromaffin Cells.......71

4.2 Priming by Munc13s in the Context of Vesicle Pool Models ....................................73

4.3 Factors That May Mediate Residual Release in Munc13-1/2 DKO Cells .............75

4.4 Possible Mechanisms of Munc13 Function...................................................................76

4.4.1 Facilitation of SNARE Complex Formation and Tethering of Vesicular Membranes ...............................................................................................................76

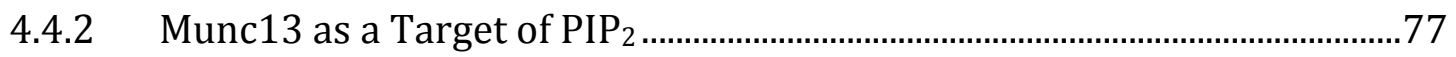

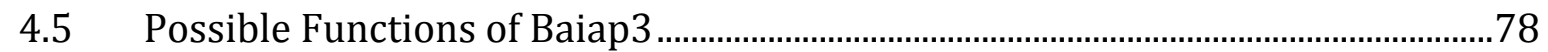

4.5.1 Possible Functions of Baiap3 in the Adrenal Medulla...................................79

4.5.2 Possible Functions of Baiap3 in the Hypothalamus and Amygdala ..........79

4.6 The Choice of Munc13 Isoforms as Priming Factors in Chromaffin Cells and

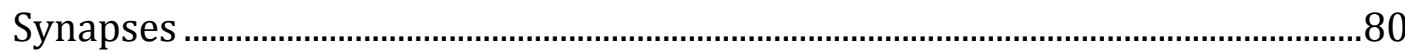

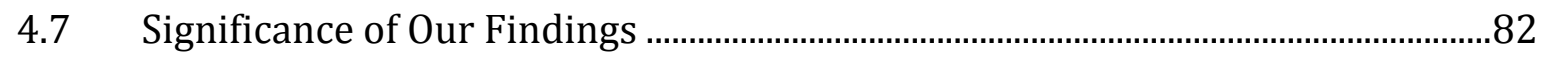

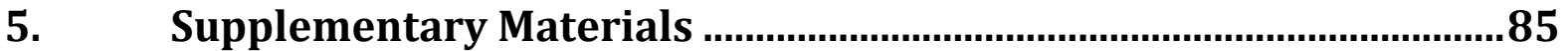

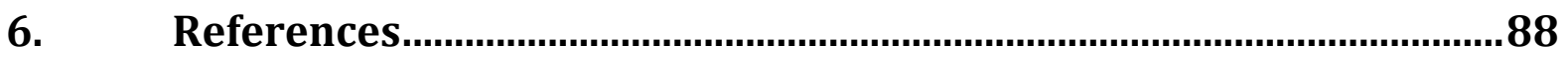

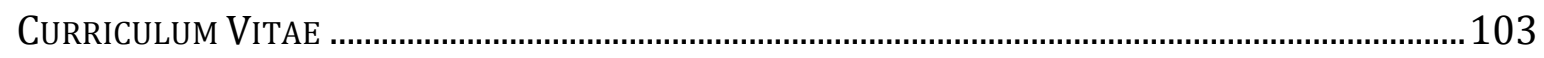




\section{Abstract}

The process of vesicular exocytosis is a fast, tightly regulated and $\mathrm{Ca}^{2+}$-triggered event mediated by SNARE complex formation. Although it is known that factors like Munc18, Munc13 and CAPS are important in setting up the SNARE core fusion machinery in a process called priming, the underlying molecular mechanism is not well understood. Munc13 is of critical importance in priming synaptic vesicles (SVs) for release, and SV exocytosis in hippocampal synapses is completely shut down in the absence of Munc13-1 and Munc13-2, whereas in the case of synaptic dense core vesicles (DCVs) the synaptic preference of DCV release is lost. CAPS was originally identified as a factor which reconstitutes secretion in permeabilised neuroendocrine cells, and has since been recognised as important in regulated release of DCVs in C. elegans and large dense core vesicles (LDCVs) in neuroendocrine chromaffin cells, as well as in SV exocytosis in neurons. Although overexpression studies had also implicated Munc13s in LDCV release in chromaffin cells, in this cell type no LDCV release deficit had ever been demonstrated in their absence, and CAPSs proteins had been suggested to be the main regulators of LDCV exocytosis. To gain a more complete picture of potential differences in the regulation of SV and LDCV exocytosis, we investigated the role of different Munc13 isoforms in chromaffin cell LDCV exocytosis. This study is the first to report a deficit in chromaffin cell LDCV exocytosis in the absence of Munc13 isoforms. The ubiquitous (ub)Munc13-2 is the dominant isoform in murine chromaffin cells, and its deletion results in reductions of $60 \%$ of the fast burst component, of $52 \%$ of the slow burst component and of $72 \%$ of the sustained component, which is a more drastic reduction of release than in chromaffin cells of CAPSdeficient mice. Munc13-1 expression is low in perinatal adrenal glands, and its deletion alone did not result in significant changes in exocytosis, however its function in LDCV release became apparent in the absence of Munc13-2. Munc13-1 appears to mostly contribute to the slow burst and sustained component of release. By contrast, deletion of Baiap3, another Munc13 isoform with relatively high expression in adrenal medulla, did not lead to changes in LDCV exocytosis and its overexpression could not rescue the release deficit of Munc131/2 deficient cells. The remaining Munc13 isoforms, bMunc13-2, Munc13-3 and Munc13-4 are not expressed in perinatal adrenal glands and do not contribute to LDCV exocytosis in this cell type. Taken together, our findings show that ubMunc13-2 and Munc13-1 regulate LDCV exocytosis in chromaffin cells. Thus, at least in mammals, both Munc13s and CAPS 
proteins are critical in the regulation of both SV and LDCV exocytosis in neurons as well as in neuroendocrine cells. 


\section{Introduction}

\subsection{The Brain, Neurons and Synapses}

The brain is the central organ for information processing in humans. The human brain contains $\approx 10^{11}$ neurons which form an interconnected network enabling the reception and processing of information as well as the execution of responses. A typical neuron comprises four functional regions, namely a cell body, an axon, several dendrites and a pre-synaptic terminal. Dendrites can arborize and specialises in receiving signals from other neurons. The axon extends from the cell body to send electrical signals to other neurons. A typical neuron has a resting membrane potential of $-60 \mathrm{mV}$ to $-70 \mathrm{mV}$. The resting membrane potential is maintained by the $\mathrm{Na}^{+} / \mathrm{K}^{+}$-ATPase and the fact that ion channels that span the neuronal plasma membrane are more permeable to $\mathrm{K}^{+}$than to $\mathrm{Na}^{+}$at the resting potential. Voltagegated channels respond to changes in membrane potential by a conformational change, opening the channels and allowing for ion flux that is specific for the channel and in accordance to the electrochemical gradient across the membrane. When the membrane is depolarised to above a certain threshold, an action potential is generated which propagates along the axon. When the action potential reaches the pre-synaptic terminal of a chemical synapse, it causes the opening of voltage-gated $\mathrm{Ca}^{2+}$ channels and results in $\mathrm{Ca}^{2+}$ influx into the pre-synapse. This leads to an increase in pre-synaptic $\left[\mathrm{Ca}^{2+}\right]$ and triggers the fusion of synaptic vesicles with the plasma membrane, releasing their content, neurotransmitter, into the synaptic cleft. Neurotransmitter molecules traverse the synaptic cleft and bind to postsynaptic receptors, including ligand-gated ion channels. These channels then open or close, and thus the electrical signal is propagated to the post-synaptic cell (Kandel et al., 2013).

The excitation-secretion-coupling in synapses is extremely efficient, with $\mathrm{Ca}^{2+}$ triggered vesicle fusion lagging behind the influx of $\mathrm{Ca}^{2+}$ by a mere $60 \mu \mathrm{s}$ (Sabatini and Regehr, 1996). This efficiency is crucial for the ability of neurons to encode and compute information in neural circuits, for example via processes such as short-term synaptic plasticity (Abbott and Regehr, 2004). The maintenance of the extremely short delay relies on a fusion machinery that is highly specialised for fast and synchronised release. The soluble $N$ ethylmaleimide-sensitive factor attachment protein receptor (SNARE) proteins constitute the core fusion machinery of pre-synaptic vesicle release. 


\subsection{Synaptic Vesicle Cycle}

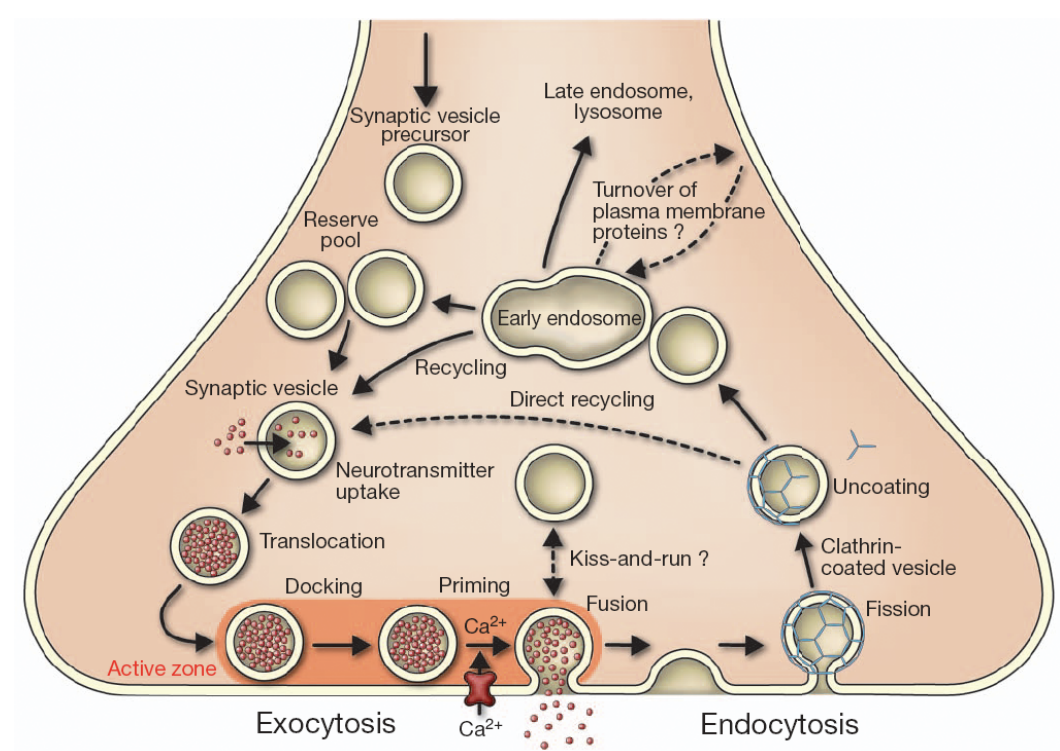

Fig.1.1 The synaptic vesicle cycle (Jahn and Fasshauer, 2012).

The synaptic vesicle (SV) cycle is shown in Fig. 1.1. Synaptic vesicle precursors (SVP) are synthesised in the cell soma. They are transported anterogradely along the axon to the synaptic domain of the neuron by microtubule-dependent trafficking mediated by the kinesin family of proteins (Maeder et al., 2013). A reserve pool of SVs is maintained in the active zone for mobilisation on demand (Denker and Rizzoli, 2010). This pool is thought to be maintained in part by synapsins, which tether SVs to each other and to actin, but are able to dissociate from both upon phosphorylation (Fornasiero et al., 2012). During maturation, SVPs acquire the correct complement of membrane proteins and neurotransmitters are filled into SVs at the pre-synapse. Mature SVs are then translocated to the site of neurotransmitter release, the active zone (AZ), where they are docked and primed. Docking refers to the process by which secretory vesicles are rendered in contact with or in very close proximity to the plasma membrane and is based on morphological data obtained by electron microscopy (EM) (Verhage and Sorensen, 2008). Priming is the process by which vesicles attain a state which enables them to be released without further maturation steps at the arrival of the $\mathrm{Ca}^{2+}$ signal, and is based on physiological data. Priming is thought to occur after docking, but whether they represent partially overlapping steps or even the same step remains disputable. When the pre-synaptic membrane is depolarised, voltage-gated $\mathrm{Ca}^{2+}$ channels open and $\mathrm{Ca}^{2+}$ influx occurs down the electrochemical gradient across the membrane. $\mathrm{Ca}^{2+}$ ions bind to $\mathrm{Ca}^{2+}$ sensor proteins, e.g. synaptotagmins, which undergo conformational changes leading to 
fusion of SVs with the plasma membrane. After fusion, endocytosis takes place in the vicinity of AZs for membrane retrieval.

\subsection{Vesicle Pool Dynamics}

The concept of the existence of dynamic vesicle pools is useful for explaining diverse physiological phenomena of SV release, including short-term plasticity (Zucker and Regehr, 2002). The total number of SVs contained per synapse in hippocampal excitatory neurons is estimated morphologically to be around 100-200 (Schikorski and Stevens, 1997). The classical model of synaptic vesicle pools contains the readily releasable pool (RRP), the recycling pool (RP) and the resting pool $\left(\mathrm{R}_{t} \mathrm{P}\right)$, the last of which is alternatively known as the reserve pool (Alabi and Tsien, 2012; Denker and Rizzoli, 2010). The RRP consists of vesicles that are ready to undergo fusion once the $\mathrm{Ca}^{2+}$ signal arrives. Thus, they have the highest release probability and are thought to be docked and primed at the AZ. The RRP consists only of 5-9 SVs in a hippocampal bouton (Murthy and Stevens, 1999) and is depleted in a few seconds with 10-40 Hz stimulation (Stevens and Williams, 2007). The number of RRP vesicles can be estimated by application of hypertonic sucrose $(0.5 \mathrm{M}, 5 \mathrm{~s})$ (Rosenmund and Stevens, 1996). This method, although non-physiological, has the advantage over the use of action potential-triggered release that the mechanism of hyperosmotic release is not $\mathrm{Ca}^{2+}$-dependent (Geppert et al., 1994; Rosenmund and Stevens, 1996), thus circumventing the interference of the resting RRP size by concurrent $\mathrm{Ca}^{2+}$ accelerated vesicle replenishment into the RRP (Smith et al., 1998). The size of the RRP and the vesicular release probability of SVs in the RRP have an impact on the efficiency of neurotransmission. After depletion of the RRP, the RP is released. The size of the RP is typically around 3 times that of the RRP (Murthy and Stevens, 1999). During high frequency stimulation, the RP is released with a time constant slower than the RRP, indicating that vesicles in the RP need to undergo further maturation steps before becoming releasecompetent. The transitions for RP vesicles to the release-ready state provide opportunities for regulation (Alabi and Tsien, 2012). Replenishment from the RP to the RRP is accelerated not only by activity-dependent increases in intracellular $\mathrm{Ca}^{2+}$ but can also be enhanced by protein kinase $\mathrm{C}$ activation (Stevens and Sullivan, 1998). The $\mathrm{R}_{\mathrm{t}} \mathrm{P}$ represents a pool that remains release-reluctant during prolonged stimulation when vesicle turnover is already maximal (Harata et al., 2001; Li et al., 2005), although evidence had been presented to show 
that in some preparations (e.g. the frog neuromuscular junction), using physiological but prolonged stimulation, all vesicles participate in release (Betz and Henkel, 1994; Ceccarelli et al., 1972). The $\mathrm{R}_{\mathrm{t}} \mathrm{P}$ pool constitutes around $50-85 \%$ of all vesicles (Fernandez-Alfonso and Ryan, 2008; Harata et al., 2001), and can be mobilized by cellular signalling pathways, such as protein kinase A stimulation (Kuromi and Kidokoro, 2005) and cyclin-dependent kinase (CDK5) inhibition (Kim and Ryan, 2010). The RP and the $\mathrm{R}_{t} \mathrm{P}$ are thought to be spatially intermixed throughout the synaptic bouton (Harata et al., 2001; Rizzoli and Betz, 2005). It is suggested that newly endocytosed vesicles belonged to the RP and have high mobility, which contributes to their relative readiness for release (Denker and Rizzoli, 2010; Kamin et al., 2010). In the absence of stimulation, these vesicles integrate into the $\mathrm{R}_{t} \mathrm{P}$ (Rizzoli and Betz, 2004) and become more immobile, thus more reluctant to release (Kamin et al., 2010). Modulation of pool sizes and dynamics of conversions between pools affect synaptic reliability and synaptic strength (Alabi and Tsien, 2012).

\subsubsection{Vesicle Pool Dynamics in Chromaffin Cells}

The adrenal medullary chromaffin cell is a well characterised model system for studying neurosecretion. Chromaffin cells contain large dense core vesicles (LDCVs), and secrete catecholamines, which mediate the fight or flight response and are involved in a variety of stress and anxiety-related responses. In chromaffin cells, vesicular exocytosis, which causes an increase in the membrane surface area, can be monitored by capacitance measurement across the surface of the whole cell at high time resolution (Neher and Marty, 1982). Carbon fibre amperometry, which measures the amount of oxidisable secretion electrochemically, is often used simultaneously alongside capacitance measurements to indicate the secretion of oxidisable catecholamines (Bruns, 2004). Amperometry is also useful in measuring fusion pore dynamics and detecting the release of single vesicles.

Various vesicle pools had been identified in chromaffin cells. Vesicle pools can be identified using a strong stimulus that depletes the RRP with such fast kinetics that enables its isolation from slow replenishment processes (Sorensen, 2004). Flash photolysis of a photolabile $\mathrm{Ca}^{2+}$-chelator can be used to achieve a sudden homogeneous increase of $\left[\mathrm{Ca}^{2+}\right]$ throughout the cell from hundreds of nanomolar to tens of micromolar $\left[\mathrm{Ca}^{2+}\right]$ (Naraghi et al., 1998). This increase in $\left[\mathrm{Ca}^{2+}\right]$ is independent of $\mathrm{Ca}^{2+}$ channels and thus exocytosis triggered with this method does not reflect the localisation of vesicles with respect to $\mathrm{Ca}^{2+}$ channels but 
the kinetic characteristics inherent to vesicle pools. Analysis of release kinetics showed, that within $1 \mathrm{~s}$ of the flash, a sum of two exponentials was necessary to fit the membrane capacitance increase, indicating that two kinetically distinct pools of vesicles exist (Xu et al., 1998). The faster pool is released with a time constant of $\approx 20-40 \mathrm{~ms}$ at $\left[\mathrm{Ca}^{2+}\right]=20 \mu \mathrm{M}$ and is regarded as the RRP, while the slower pool (slowly-releasable pool, SRP) is released with a time constant of $\approx 200-400 \mathrm{~ms}$ at $\left[\mathrm{Ca}^{2+}\right]=20 \mu \mathrm{M}$ (Sorensen, 2004). Together, these two pools are termed the exocytotic burst. After depletion of this burst, further slower release (socalled sustained release), which has a near constant rate of release, ensues. The difference in release kinetics of the pools is thought to indicate the degree of maturation of the different pools. The sizes of the RRP and the SRP show a biphasic relationship with pre-flash $\left[\mathrm{Ca}^{2+}\right]$ (Fig. 1.2A-C), with maximal pool sizes at $\approx 600 \mathrm{nM}$ of pre-flash $\left[\mathrm{Ca}^{2+}\right]$, equivalent to around 140 vesicles per pool (Voets, 2000) assuming that the release of one vesicle causes a capacitance increase of $1.3 \mathrm{pF}$ (Moser and Neher, 1997). Assuming a parallel pool model (PPM) of release (Fig. 1.3B), in which both the RRP and SRP are releasable but each with a distinct $\mathrm{Ca}^{2+}$ sensor requiring the binding of three $\mathrm{Ca}^{2+}$ ions to trigger release, the transition from the SRP to the RRP is thought to be $\mathrm{Ca}^{2+}$-independent due to the unaltered contribution of the two pools to the burst at pre-flash intracellular $\left[\mathrm{Ca}^{2+}\right]$ under $600 \mathrm{nM}$ (Fig. 1.2D) (Voets, 2000). Beyond this range, the exocytotic burst decreases in size with increasing $\left[\mathrm{Ca}^{2+}\right]$ due to loss of vesicles through exocytosis. In the same study, the authors determined that both the size of the exocytotic burst and the fraction of RRP to the burst are not affected by post-flash $\left[\mathrm{Ca}^{2+}\right]$. Recently, it has been suggested that an alternate model of release, the sequential pool model (SPM) (Fig. 1.3A), can recapitulate release from chromaffin cells as well as or better than the PPM (Walter et al., 2013). In particular, the SPM can more parsimoniously simulate the faster recovery of the RRP triggered by a second flash stimulus after depletion of both pools using a first flash (Pinheiro et al., 2013). This model also describes the concurrent increase in the fast time constant of release and proportional shift of the SRP to the RRP detected in a synaptobrevin-2 mutant with defective C-terminal interaction sites (Walter et al., 2010). The SPM further predicts the existence of a $\mathrm{Ca}^{2+}$ dependent catalytic step, which endows a pool of non-releasable vesicles with RRP status, with the RRP being the only releasable pool of vesicles in this model. Interestingly, the authors found that if the PPM was modified to incorporate a $\mathrm{Ca}^{2+}$-dependent catalytic step for the SRP to RRP conversion, the PPM can also account for the fast recovery in the dual-flash protocol (Walter et al., 2013). 

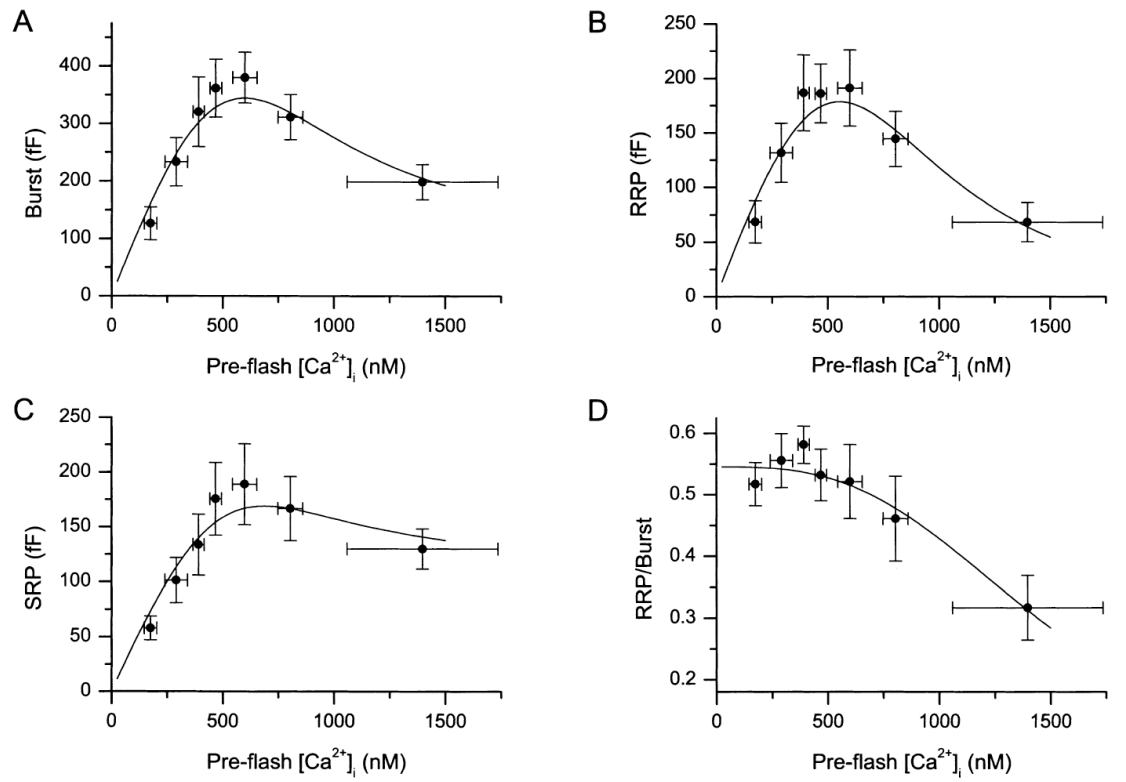

Fig. 1.2 $\mathrm{Ca}^{2+}$-dependence of the (A) exocytotic burst, (B) RRP, (C) SRP and the (D) contribution of the RRP to the exocytotic burst in chromaffin cells (Voets, 2000).

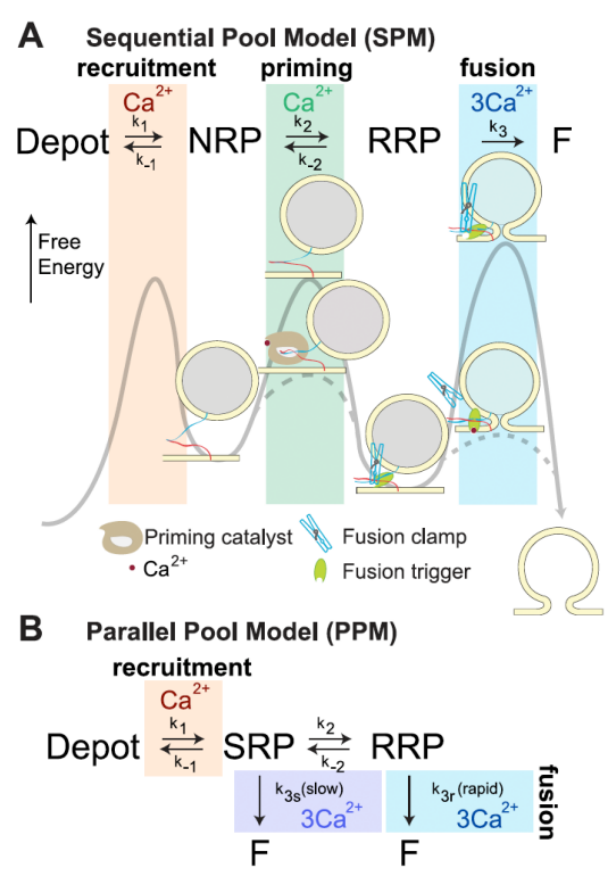

Fig. 1.3 (A) Sequential and (B) parallel models of secretion (Walter et al., 2013).

Because in $\mathrm{Ca}^{2+}$ uncaging experiments the post-flash $\left[\mathrm{Ca}^{2+}\right]$ levels can not only trigger exocytosis, but at $\left[\mathrm{Ca}^{2+}\right]$ of $>50 \mu \mathrm{M}$ elicit rapid endocytosis (Sorensen, 2004), and at concentrations of $>100 \mu \mathrm{M}$ result in the release of an "intermediate" pool of vesicles which do not contain catecholamines (Xu et al., 1998), an accurate control of both pre- and post- 
flash $\left[\mathrm{Ca}^{2+}\right]$ is required for studying the exocytosis of catecholamine-containing vesicles from chromaffin cells.

Unlike $\mathrm{Ca}^{2+}$ uncaging by flash photolysis, short trains of depolarisations cause high local $\left[\mathrm{Ca}^{2+}\right]$ in close proximity to $\mathrm{Ca}^{2+}$ channels, referred to as $\mathrm{Ca}^{2+}$ microdomains (Neher, 1998). The high $\left[\mathrm{Ca}^{2+}\right]$ is sensed by vesicles co-localised with $\mathrm{Ca}^{2+}$ channels and they are consequently rapidly released. This pool of vesicles is known as the "immediately releasable pool" (IRP) of vesicles (Horrigan and Bookman, 1994; Voets et al., 1999). Using crossdepletion experiments, it had been shown that the IRP is a component of the RRP and represents around 25\% of RRP vesicles (Voets et al., 1999).

\subsection{Comparison between Synaptic Vesicle Exocytosis and Large Dense Core Vesicle Exocytosis}

Numerous features distinguish SV and LDCV exocytosis from each other. The distinct modes of biogenesis and difference in release kinetics between these two types of organelles imply that the molecular machineries underlying vesicle delivery to the plasma membrane and fusion of these two types of vesicles might be different (Xu and $\mathrm{Xu}, 2008)$.

SVs contain neurotransmitters such as glutamate, $\gamma$-aminobutyric acid (GABA), glycine, acetylcholine and serotonin. Their diameters are around $50 \mathrm{~nm}$ (Kriebel et al., 1986) and they appear as small clear vesicles in electron microscopy. In contrast, LDCVs contain mainly neuropeptides and hormones, are 100-300 nm in diameter (Grabner et al., 2006) and are observed as electron dense organelles. In synapses the dense core vesicle (DCVs) diameter measures around $70 \mathrm{~nm}$ (Fig. 1.4). They can also be observed at low numbers in the post-synapse (van de Bospoort et al., 2012). 


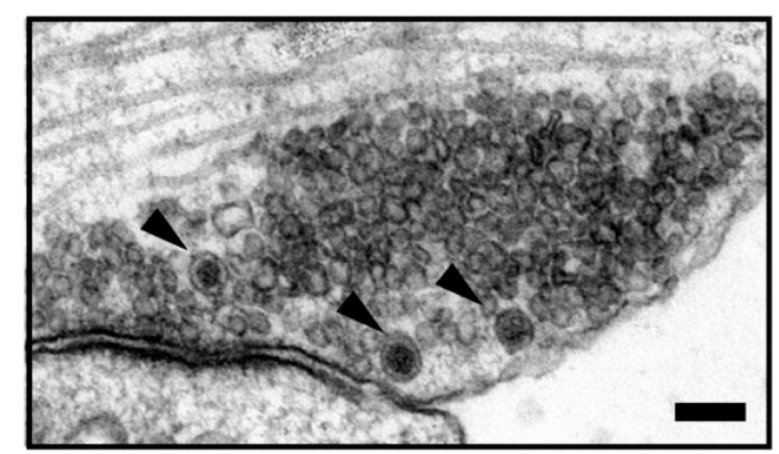

Fig. 1.4 Dense core vesicles and synaptic vesicles (SV) in hippocampal synapses. SV clusters can be seen. Arrowheads indicate DCVs. Scale bar, $100 \mathrm{~nm}$ (van de Bospoort et al., 2012).

\subsubsection{Biogenesis of SVs and LDCVs}

A large number of neuropeptides and other cargo are packaged into each LDCV in a highly condensed manner to ensure efficient storage and osmotic inertness $(\mathrm{Xu}$ and $\mathrm{Xu}$, 2008). Immature LDCVs are generated de novo by budding at the trans-Golgi network (TGN) (Santos et al., 2009). The granin family of proteins, e.g. chromogranins and secretogranins, are secreted together with neuropeptides from LDCVs and are thought to facilitate budding from the TGN (Xu and $\mathrm{Xu}, 2008)$. Granins bind $\mathrm{Ca}^{2+}$ with low affinity. $\mathrm{Ca}^{2+}$ binding leads to aggregation of granins which induces wrapping of the TGN membrane around the aggregates, causing membrane deformation and budding ( $\mathrm{Xu}$ and $\mathrm{Xu}, 2008$ ). The role of granins in LDCV biogenesis is exemplified by the reduced number of LDCVs in PC12 cells (a cell line derived from rat pheochromocytoma, a cancer of the adrenal medulla) following chromogranin A downregulation and formation of LDCV induced by overexpression of chromogranin A in fibroblasts (Kim et al., 2001). Immature LDCVs undergo homotypic fusion that requires syntaxin-6 and synaptotagmin IV. In PC12 cells, synaptotagmin IV depletion inhibits homotypic fusion and results in decreased endoproteolytic activation of prohormone convertase 2, which is required for processing of secretogranin II, a DCV cargo (Ahras et al., 2006). Missorted proteins are removed by budding, for example, using adaptor protein (AP)-1/clathrin-coated vesicles (Klumperman et al., 1998). Neuronal adaptor protein-3 (AP-3), a multi-subunit protein, pinches off smaller vesicles from the larger immature vesicles during maturation, thus regulating the sizes and quantal content of LDCVs in chromaffin cells (Grabner et al., 2006). In addition, Rab3, a 
vesicular GTPase that has been identified with a role in tethering vesicles, appears to be important in vesicle biogenesis in chromaffin cells, as the total number of LDCVs per cell section was halved in mice lacking all four Rab3 isoforms (Schonn et al., 2010). LDCVs are replenished by KIF-1A-dependent anterograde transport of granules generated from the trans-Golgi network (Zahn et al., 2004). Furthermore, single LDCVs can also undergo secretion without releasing all of their contents before re-sealing to form a granule (Perrais et al., 2004). In hippocampal neurons, only $20 \%$ of DCVs were observed at synapses, while $65 \%$ of release occurred at synaptic sites, which led to the suggestion that neuronal DCVs are mobilised to release sites during activity, where they are then docked and primed for release (van de Bospoort et al., 2012).

Unlike the LDCVs, SVs are supplied locally near their site of release, the AZ, so that high supply rates can be sustained during high synaptic activity. SV proteins are thought to be synthesised in the cell soma and transported to nerve terminals by membrane carriers via fast axonal transport along microtubules (Santos et al., 2009). They may be delivered to the plasma membrane and undergo series of exo-endocytic cycles, during which they become sorted into SVs (Santos et al., 2009). After fusion, membrane retrieval occurs by endocytosis near the AZ. Clathrin-mediated endocytosis represents a major pathway of endocytosis. Membrane invagination at the outer margins of the AZ causes the appearance of clathrincoated buds, and fission is mediated by the GTPase dynamin (Conner and Schmid, 2003). The clathrin coat is composed of an outer layer of clathrin and an inner layer of adaptor proteins, such as adaptor protein-2 (AP-2) (Saheki and De Camilli, 2012). Uncoating of the clathrin and adaptor coat occurs rapidly after fission, is ATP-dependent, and requires Hsc70 ATPase and its cofactor auxilin (Yim et al., 2010). Uncoated vesicles are rapidly recruited into SV clusters for re-use (Saheki and De Camilli, 2012). SVs are also retrieved through bulk endocytosis, followed by the formation of endocytic vacuoles which subsequently generate SVs through unknown mechanisms. Lastly, SVs are proposed to be recycled through "kiss-and-run", in which a transient fusion pore forms and close rapidly without collapse of the vesicle into the plasma membrane (Saheki and De Camilli, 2012).

\subsubsection{Docking of SVs and LDCVs}

Different criteria of docking can be employed by investigators, depending on the techniques and biological systems used, as well as the degree of stringency deemed 
appropriate for the study. Some authors consider vesicles located within a certain distance (e.g. $30 \mathrm{~nm}$ ) from the plasma membrane as docked, while others count only vesicles forming a "contact patch" with the plasma membrane as docked (Verhage and Sorensen, 2008). Various proteins have been implicated in the docking of SVs and LDCVs. Munc18 is required for docking of both SVs and LDCVs. Chromaffin cells from Munc18 null mutant mice show a 10-fold reduced secretion and a concomitant reduction in the number of morphologically docked LDCVs (Voets et al., 2001). Mice heterozygous for Munc18 show $30-50 \%$ reduction in the number of docked SVs (Toonen et al., 2006b) in autaptic hippocampal neurons and in docked LDCVs in chromaffin cells (Toonen et al., 2006a). In unc-18 mutant nematodes the number of docked SVs is reduced to $36 \%$ of wild-type at neuromuscular synapses (Weimer et al., 2003). Thus, Munc18 is an established docking factor for both LDCVs and SVs.

Munc18 interact closely with syntaxin-1, both in a binary complex and when syntaxin-1 is in the SNARE complex. Botulinum neurotoxin $\mathrm{C}$ (BoNT/C) cleavage of syntaxin-1 in chromaffin cells results in a strong reduction of the number of docked LDCVs but no apparent changes in SV docking in synaptic terminals of autaptic hippocampal neurons, despite a near total abrogation of release in both cell types (de Wit et al., 2006). In agreement with this finding, the number of docked insulin granules (a type of LDCV) is decreased in pancreatic $\beta$-cells from syntaxin-1A knockout (KO) mice (Ohara-Imaizumi et al., 2007). However, using a high pressure freezing (HPF) technique, which is thought to render a more accurate picture of ultrastructural morphology, a strong docking defect was observed in both excitatory cholinergic and inhibitory GABAergic neurons of syntaxin null mutant worm at the $\mathrm{AZ}$ and peri-synaptically, showing that syntaxin is required for $\mathrm{SV}$ docking in C. elegans synapses (Hammarlund et al., 2007). The same group also reported that docking of synaptic dense core vesicles (DCV), which are similar to LDCVs and releases neuropeptides and catecholamines from neurons, is dependent on syntaxin (Hammarlund et al., 2008), corroborating previous data acquired using chemical fixation. Taking the available evidence into consideration, it may be said that syntaxin is essential for docking LDCVs onto plasma membranes, but its role in SV docking remains unclear due to conflicting results reported using different methods in different species. It is worth noting that in mice expressing open syntaxin-1B in a syntaxin-1A KO background, docking is intact for SVs in neurons but not for LDCVs in chromaffin cells, demonstrating that the Munc18/closed 
syntaxin complex is required for LDCV docking in chromaffin cells but not for SVs in synapses (Gerber et al., 2008).

Another t-SNARE protein, SNAP-25, was shown by HPF to be required for docking in both isolated chromaffin cells and intact adrenal glands (Fig. 1.5) (de Wit et al., 2009), while synaptobrevin-2 was not essential for LDCV docking in chromaffin cells (conventional fixation, (Gerber et al., 2008)). Chromaffin cells from SNAP-25 KO mice showed a less severe docking phenotype than Munc18- and syntaxin-1-deficient mice (de Wit et al., 2009). Moreover, chromaffin cells from synaptotagmin-1 null mice exhibited a strong docking defect in the 2009 study (de Wit et al., 2009). Contrary to the standard SNARE hypothesis of vesicle docking and fusion where synaptotagmin binds to the assembled SNARE complex (Sollner et al., 1993a), it was thus proposed that synaptotagmin-1 is the vesicular protein that mediates the interaction with the syntaxin-1/SNAP-25 acceptor complex to accomplish docking (de Wit et al., 2009). Another study showed that synaptobrevin cleavage by botulinum neurotoxin D (BoNT/D) perturbed LDCV docking in live PC12 cells as measured by total internal fluorescence microscopy (TIRF) and EM of cells fixed using HPF (Wu et al., 2012). The authors of this study described that the N-terminus of synaptobrevin was important for docking, and thus proposed that N-terminal assembly of trans-SNARE proteins underlies docking. In addition to this, Rab3 GTPases have also been implicated in LDCV docking. Overexpression of Rab3A in chromaffin cells increases docking by $\approx 60 \%$ (van Weering et al., 2007). However, in chromaffin cells deficient for all four isoforms of Rab3, the total number of vesicles per cell section was reduced by $\approx 50 \%$, but no changes were observed in the number of docked vesicles (Schonn et al., 2010). Another Rab GTPase, Rab27, and its effector granuphilin, may mediate LDCV docking in multiple systems, including PC12 cells (Tsuboi and Fukuda, 2006), pancreatic $\beta$-cells (Kasai et al., 2005) and melanosomes (Fukuda, 2006). 

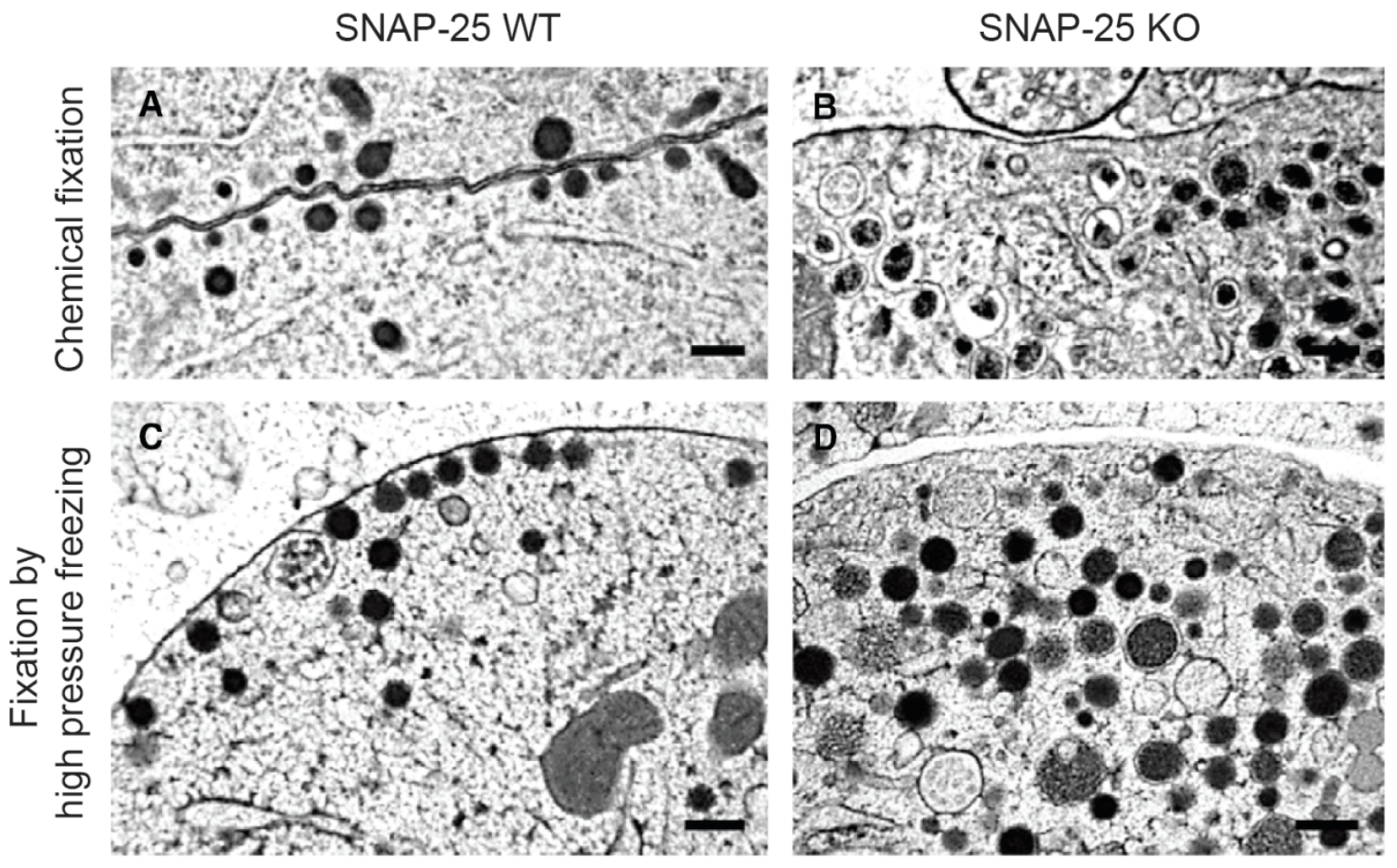

Fig. 1.5 Docking deficit in SNAP-25 KO chromaffin cells as an example of a docking phenotype. (A-B) Chemically fixed chromaffin cells from SNAP-25 WT and KO cells. (C-D) Fixation by high pressure freezing in SNAP-25 WT and KO cells (modified from (de Wit et al., 2009).

Munc13s and CAPS have been established as priming factors for SVs (Augustin et al., 1999b; Varoqueaux et al., 2002) and LDCVs (Liu et al., 2010; Zhou et al., 2007), respectively. The contributions of Munc13s and CAPS in docking these two types of vesicles will be discussed in the sections "Role of Munc13 Isoforms in Priming and Docking" and "Role of CAPS in Priming and Docking".

\subsection{Mechanisms of Fast Vesicular Exocytosis}

\subsubsection{SNARE Proteins as the Core Fusion Machinery}

Fusion of SVs with the pre-synaptic plasma membrane is an extremely fast and tightly regulated membrane fusion event (Wojcik and Brose, 2007). The excitation-secretion coupling occurs within $1 \mathrm{~ms}$ (Sabatini and Regehr, 1996) and is strictly dependent on the intracellular $\left[\mathrm{Ca}^{2+}\right]$. Although SV fusion events, like other cellular membrane fusion events, are dependent on SNARE proteins, a specialised set of regulatory proteins are required for fusion at this extraordinary speed and for the tight coupling to $\mathrm{Ca}^{2+}$ influx. 
The importance of SNARE proteins in vesicular fusion was realised when their proteolytic cleavage by clostridial neurotoxins was found to block different kinetic components of release (Banerjee et al., 1996; Capogna et al., 1997; Lawrence et al., 1997; McMahon et al., 1992). In chromaffin cells, botulinum neurotoxin (BoNT) serotype E, which cleaves the terminal 26 amino acids of SNAP-25, completely blocked all components of release induced by uncaging of $\mathrm{Ca}^{2+}$, while BoNT/A, which cleaves 17 amino acids upstream, reduced mainly the slower component of release and caused a slower release rate of the faster component, the exocytotic burst (Xu et al., 1998). BoNT/C, which cleaves SNAP-25 at a site one amino acid downstream of the BoNT/A cleavage site and also cleaves syntaxin, caused a complete block of all release components, indicating that syntaxin-1 is essential for exocytosis in chromaffin cells. Finally, tetanus toxin (TeNT) and BoNT/D, which cleaves synaptobrevin, also reduced all components of release (Xu et al., 1998). These studies thus highlight the essential role of SNARE proteins in exocytosis in neurosecretory cells.

In neurons and chromaffin cells, the SNARE proteins syntaxin-1, SNAP-25 and synaptobrevin-2 constitute the core fusion machinery. SNARE proteins are mechanistically highly conserved through evolution and are characterised by the presence of a SNARE motif, which forms an $\alpha$-helix (Kloepper et al., 2007). The SNARE motifs of Syntaxin-1, SNAP-25 and synaptobrevin-2 readily form a parallel four-helix bundle, which forms the core of a stable heterotrimeric complex called the SNARE complex (Fig. 1.6). The four-helix bundle is arranged into 16 layers contributed by highly conserved amino acid side chains facing the core of the bundle (Fasshauer et al., 1998). Syntaxin-1 and SNAP-25 are known as t(target)SNAREs because of their plasma membrane localisation, while synaptobrevin-2 is known as the v(vesicular)-SNARE because of its vesicular localisation (Rothman, 1994; Sollner et al., 1993b). SNAREs can also be classified into R-SNAREs (e.g. synaptobrevin-2) or QSNAREs (e.g. SNAP-25, syntaxin-1) according to the amino acid (arginine and glutamine, respectively) that they contribute at the polar layer 0 of the core of the SNARE complex (Fasshauer et al., 1998). Both syntaxin-1 and synaptobrevin-2 contribute one SNARE motif, while SNAP-25 contributes two SNARE motifs to the four-helix bundle. 


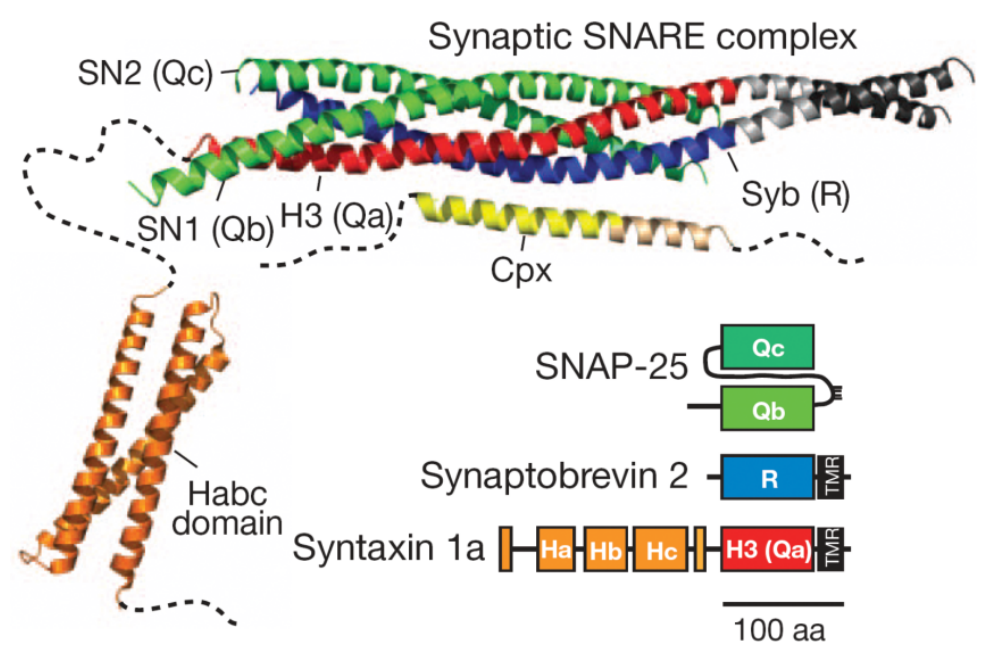

Fig. 1.6 Structure of the SNARE complex and the domain structures of three SNARE proteins SNAP-25, syntaxin-1 and synaptobrevin-2 (modified from (Jahn and Fasshauer, 2012)).

Both syntaxin-1 and synaptobrevin-2 have transmembrane regions (TMRs) at their Cterminal region, which anchor them in the membrane (Fig. 1.6). In contrast, SNAP-25 lacks a TMR and is anchored in the plasma membrane via palmitoylated cysteine residues at the linker between its two SNARE motifs. Apart from the TMR and the SNARE motif (also called the H3 domain), syntaxin-1 harbours also an autonomously-folding antiparallel threehelix bundle called the Habc domain (Fernandez et al., 1998; Lerman et al., 2000). This three-helix bundle folds back onto the syntaxin-1 SNARE motif to form the closed conformation of syntaxin-1. In this conformation, access to the syntaxin-1 SNARE motif is limited and core complex formation cannot take place (Dulubova et al., 1999; Misura et al., 2000). Syntaxin-1 contains also the N-peptide, which is situated at the very end of the Nterminus, and is important for interaction with Munc18 (Dulubova et al., 2007; Khvotchev et al., 2007; Shen et al., 2010). Structurally, syntaxin exhibits alpha-helicity, while SNAP-25 and synaptobrevin are largely unstructured, and are folded upon contact with their binding partners when they form the SNARE complex (Fasshauer et al., 1997; Wiederhold and Fasshauer, 2009).

Membrane fusion requires a considerable amount of energy because two negatively charged and inherently stable membranes have to be brought together. The "zippering" of SNARE motifs from the membrane-distal N-termini towards the membrane-proximal Ctermini into a four-helix bundle is highly exergonic and is thought to provide the energy for bringing the vesicular membrane and the plasma membrane into close apposition and for initiating fusion of both lipid bilayers (Hanson et al., 1997; Pobbati et al., 2006). In a study 
using surface forces apparatus (SFA) in which two lipid bilayers each reconstituted with neuronal t- or v-SNARE proteins were allowed to approach each other, it was estimated that SNARE complexes started to form when the bilayers were around $8 \mathrm{~nm}$ apart and at this distance was able to form stable complexes in tens of seconds (Li et al., 2007). SNARE complex assembly is thought to start with the formation of a syntaxin-1/SNAP-25 heterodimer which is called an acceptor complex. Addition of synaptobrevin to this preformed acceptor complex accelerated SNARE complex formation when compared to sequential addition of the three SNARE proteins (Pobbati et al., 2006). The N-terminal of the acceptor complex provides a binding site for the binding of the N-terminus of synaptobrevin, and SNARE zippering follows to form the trans-SNARE complex. This site can be stabilised by a C-terminal synaptobrevin fragment which prevents the association of a second syntaxin1 molecule to the acceptor complex. Li et al. showed that the SNARE complex formed only partially, with 12-25 membrane-proximal residues still unstructured, and the authors suggest that the negative residues in this unstructured region may cooperate electrostatically with the negative membrane to prevent full SNARE complex zippering (Li et al., 2007). Because syntaxin-1 is a somewhat promiscuous protein (Rizo and Sudhof, 2012), complexes other than the 1:1 syntaxin-1/SNAP-25 acceptor complex are also possible. Syntaxin-1 also forms a 2:1 heterotrimer with SNAP-25 (Fasshauer et al., 1997), a 2:2 tetramer with the N-terminal SNARE motif of SNAP-25 (Misura et al., 2001a) and a homotetramer (Misura et al., 2001b). Thus, synaptobrevin competes with these alternative species to form the productive SNARE complex.

Whether SNARE complex formation releases sufficient energy for membrane fusion is an important question. Various groups have attempted to address this question by measuring the energy released by SNARE complex formation. Using single molecule dynamic force spectroscopy and SFA measurements, the energy released from SNARE complex formation measured around 23-35 $\mathrm{k}_{\mathrm{B}} \mathrm{T}$ (equivalent to $14-20 \mathrm{kcal} / \mathrm{mol}$ ) (Li et al., 2007; Liu et al., 2009). These measurements are close to but lower than the energy required (40-50 $\mathrm{k}_{\mathrm{B}} \mathrm{T}$ ) for the formation of stalk-like states between the two bilayers (Kozlovsky and Kozlov, 2002), although the activation energy for fusion has not been precisely determined and is in the range of 40-200 $\mathrm{k}_{\mathrm{B}} \mathrm{T}$ (van den Bogaart et al., 2010). Accordingly, the formation of only a few SNARE complexes would be sufficient for the initiation of membrane fusion. In another study using isothermal calorimetry to directly profile energy changes during progressive mixing (by injection) of SNARE proteins, the energy released was estimated to 
be about $11 \mathrm{kcal} / \mathrm{mol}$ (equivalent to $\approx 18.5 \mathrm{k}_{\mathrm{B}} \mathrm{T}$ ), which would call for a larger number of SNARE complexes to be formed for fusion to occur or be initiated. Based on a variety of methods in both in vitro and in vivo systems, including single vesicle fusion assays with planar membranes and detection of docking and fusion events by TIRF (Domanska et al., 2010), fusion assays with purified synaptic vesicles and detection of lipid- and contentmixing (van den Bogaart et al., 2010), capacitance measurement after flash photolysis of caged $\mathrm{Ca}^{2+}$ in chromaffin cells (Mohrmann et al., 2010) and re-expression of pHluorin-tagged synaptobrevin in synaptobrevin cellubrevin double-deficient hippocampal neurons to rescue exocytosis (Sinha et al., 2011), it has been suggested that at least 1 to 3 SNARE complexes are required for fusion to occur. After fusion has occurred, the SNARE complex is in the cisconfiguration and requires the AAA ATPase $N$-ethylmaleimide sensitive factor (NSF) and its cofactor $\alpha$ - and $\beta$-SNAP for dissociation, so that they can be recycled for a new round of fusion (Banerjee et al., 1996; Burgalossi et al., 2010; Littleton et al., 2001).

Although the essential role of SNARE proteins in exocytosis is undisputed, the precise reaction mechanism which takes place to execute fast exocytosis has not been clearly defined. For instance, in vitro SNARE-mediated liposome fusion lasts hours, possibly due to the formation of fusion-incompetent SNARE intermediates and other unknown factors (Pobbati et al., 2006). The fast exocytosis of vesicle content seen in vivo appears to depend on the presence of SNARE regulatory proteins such as Munc13, Munc18 and CAPS, which are also part of the vesicle fusion machinery.

\subsubsection{Munc18}

Mammalian uncoordinated-18 (Munc18) belongs to the family of Sec1/Munc18 (SM) proteins. This family of proteins is known to function in conjunction with SNARE proteins in diverse cellular membrane trafficking processes. The absence of Munc18 in mice leads to a complete block of neurotransmission (Verhage et al., 2000) and almost completely abrogates secretion from chromaffin cells (Toonen et al., 2006a). Munc18 is established as a docking factor both in autaptic neurons (Toonen et al., 2006b) and in chromaffin cells (GulyasKovacs et al., 2007). Munc18 expression levels controls the size of the RRP and synapse recovery at high activity (Toonen et al., 2006b). 
Munc18 binds tightly to the closed conformation of syntaxin-1 (Hata et al., 1993), with a $\mathrm{K}_{\mathrm{d}}$ of $\approx 1.4 \mathrm{nM}$ when the entire cytoplasmic domain of syntaxin-1 is used (Burkhardt et al., 2008). The binary Munc18/closed syntaxin-1 interaction is mediated by binding of the concave inner surface formed by domains 1 and 3a of Munc18 to the Habc domain, the connecting region and the $\mathrm{H} 3$ domain of syntaxin-1 as well as by binding of the outer surface of the Munc18 domain 1 to the syntaxin-1 N-peptide (Fig. 1.7) (Burkhardt et al., 2008). Similar modes of interactions involving multiple binding sites had been shown in other SM proteins and their cognate syntaxins (Burkhardt et al., 2008). When the N-peptide is bound by Munc18, SNARE complex formation is inhibited. Thus, the Munc18/closed syntaxin complex has been proposed to prevent non-productive or pre-mature SNARE complex formation. In addition, the reduction of syntaxin-1 protein levels by around 70\% in Munc18-1 null mutant mice (Toonen et al., 2005; Zhou et al., 2013) and the reciprocal decrease in Munc18 levels in a mutant mouse expressing open syntaxin that exhibits reduced Munc18 affinity (the syntaxin L165A/E166A, or "LE" mutant) suggest that the binary interaction may mutually protect both proteins from degradation (Gerber et al., 2008). Apart from binding to the closed formation of syntaxin-1, Munc18 also binds to the SNARE complex. It has been reported that Munc18 binds to the SNARE complex via its association with the syntaxin-1 Npeptide and Habc domain (Burkhardt et al., 2008), or to the four-helix bundle of the core complex (Dulubova et al., 2003). The former mode of interaction entails that Munc18 must dissociate from syntaxin to allow core complex formation, and rebind to the N-peptide after core complex formation. Munc18 binding to the SNARE complex probably serves a function, e.g. priming, that is distinct from that of its binding to closed syntaxin- 1 before the SNARE complex is formed. Munc18 may cooperate with the MUN domain of Munc13 proteins through weak interactions to accelerate the extraction of the syntaxin-1 SNARE motif from the closed complex to allow core complex formation (Ma et al., 2011). 


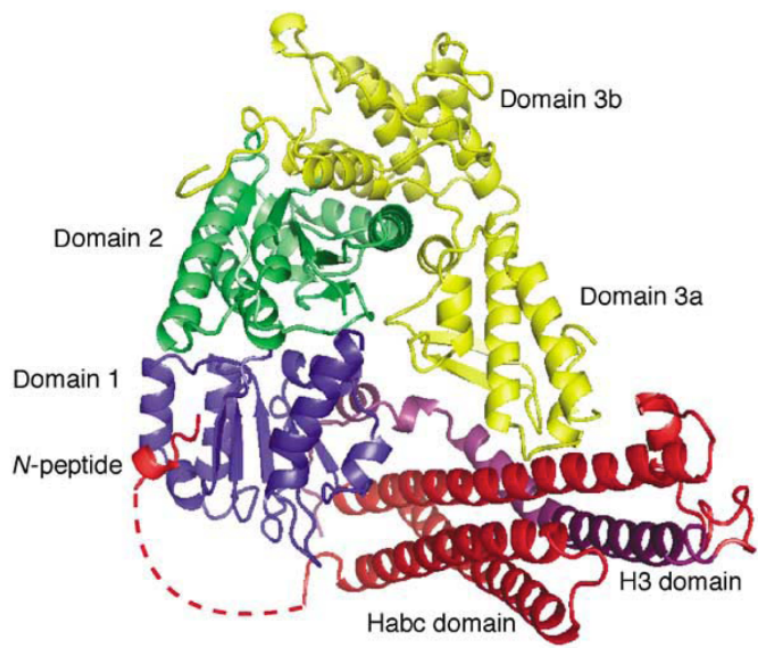

Fig. 1.7 Structure of the Munc18a-closed syntaxin1a complex (Burkhardt et al., 2008).

\subsubsection{Munc13}

Mammalian uncoordinated homology-13 (Munc13) is a family of proteins that are essential for vesicular neurotransmitter release. Mice lacking the major neuronal isoforms Munc13-1 and Munc13-2 are not viable outside the uterus. Although the brains of these mice show normal cytoarchitecture and hippocampal neurons develop normally in culture, hippocampal synapses from Munc13-1/2 double knockout (DKO) mice are devoid of both spontaneous and evoked release (Varoqueaux et al., 2002). Drosophila deficient in the expression of dunc-13, the orthologue of Munc13 in Drosophila, showed arrested synaptic transmission and an accumulation of docked vesicles at the pre-synapse (Aravamudan et al., 1999). In C. elegans, unc-13 mutants showed a drastic reduction of GABAergic and cholinergic transmission, also with accumulation of vesicles (Richmond et al., 1999). The conservation of Munc13 function across different species highlights its critical importance in synaptic transmission.

\subsubsection{Domain Structure of Munc13s}

Thus far, genes encoding five Munc13 isoforms had been identified, namely Munc131, Munc13-2, Munc13-3, Munc13-4 and brain-specific angiogenesis inhibitor-1 associated protein (Baiap3) (Augustin et al., 1999b; Betz et al., 1997; Koch et al., 2000; Shiratsuchi et al., 1998). Additionally, there are two splice variants of Munc13-2 (ubiquitous, ubMunc13-2 and brain-specific, bMunc13-2) with alternatively spliced N-termini (Betz et al., 2001). 
Munc13s are large multi-domain proteins with molecular weights ranging from $\approx 130-250$ $\mathrm{kDa}$. They share similar domain structures (Fig. 1.8) and are characterised by the presence of two C-terminal Munc13-homology domains (MHDs), MHD1 and MHD2 (Koch et al., 2000). Two $\mathrm{C}_{2}$ domains $\left(\mathrm{C}_{2} \mathrm{~B}\right.$ and $\left.\mathrm{C}_{2} \mathrm{C}\right)$, which are generally known as $\mathrm{Ca}^{2+} /$ phospholipid-binding domains, flank the region containing the MHD domains. In Munc13-1 and Munc13-2, the $\mathrm{C}_{2} \mathrm{~B}$ domain but not $\mathrm{C}_{2} \mathrm{C}$, has been shown to bind $\mathrm{Ca}^{2+}$ (Shin et al., 2010). The region between the $\mathrm{C}_{2} \mathrm{~B}$ and $\mathrm{C}_{2} \mathrm{C}$ domains (residue 849-1531), which has been termed MUN domain, folds autonomously and has been described as the minimal functional region of Munc13s (Basu et al., 2005). However, other studies identified the minimal functional region of Munc13 as a fragment that includes the MHD1, MHD2 and $\mathrm{C}_{2} \mathrm{C}$ domains (Madison et al., 2005; Stevens et al., 2005). Additional domains present in the N-termini of Munc13-1, Munc13-2 and Munc13-3 include a $\mathrm{C}_{1}$ domain, which binds diacylglycerol and phorbol esters such as $\beta$-phorbol 12,13-dibutyrate (PDBu) and phorbol myristate acetate (PMA) (Betz et al., 1998) and a conserved $\mathrm{Ca}^{2+} /$ calmodulin binding site (Junge et al., 2004; Lipstein et al., 2012). Both the $\mathrm{C}_{1}$ domain (Basu et al., 2007; Rhee et al., 2002) and $\mathrm{Ca}^{2+} /$ calmodulin-binding sites are important in the regulation of short-term synaptic plasticity characteristics of Munc13 (Junge et al., 2004; Rhee et al., 2002).

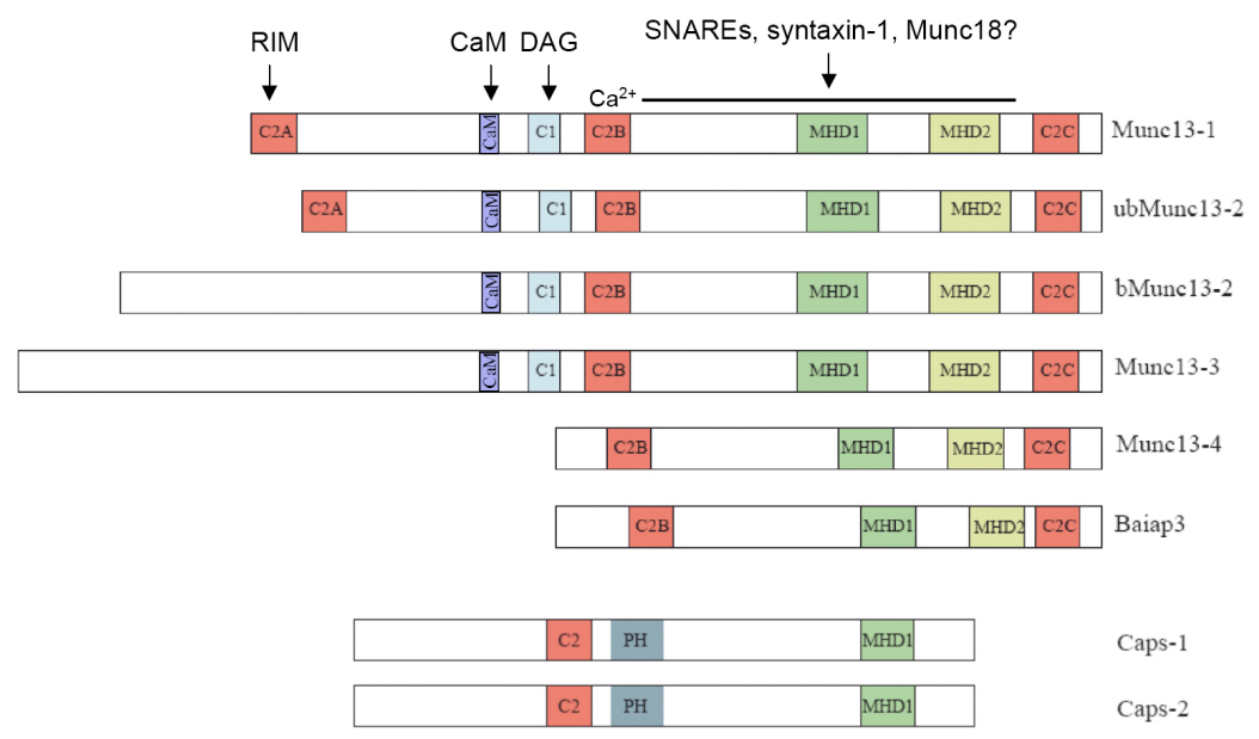

Fig. 1.8 Domain structure of Munc13 and CAPS isoforms.

The C-terminal domains of Munc13-1, -2 and -3 are highly conserved, with 75\%-85\% homology (Augustin et al., 1999b). The N-terminal regions of Munc13s are more divergent. Munc13-1 and ubMunc13-2, but not the other isoforms, possess a $\mathrm{C}_{2} \mathrm{~A}$ domain. These two isoforms form autoinhibitory homodimers through $\mathrm{C}_{2} \mathrm{~A}$ interactions, which are resolved by 
the binding of RIM to $\mathrm{C}_{2} \mathrm{~A}$ (Betz et al., 2001; Deng et al., 2011). Munc13-4 and Baiap3 are two shorter isoforms that lack the variable $\mathrm{N}$-termini but share the $\mathrm{C}_{2} \mathrm{~B}, \mathrm{MUN}$ and $\mathrm{C}_{2} \mathrm{C}$ domains with the other Munc13 isoforms. Baiap3 and Munc13-4 share 34\% homology at the amino acid sequence level (Koch et al., 2000). Weak but significant homologies had been found between the MUN domains of all Munc13 isoforms and subunits of membrane tethering complexes, e.g. Sec6, AEX-1 and Vps53, implying that MUN domains may serve tethering/docking roles similarly as proteins in other membrane fusion systems (Pei et al., 2009).

\subsubsection{Expression Pattern of Munc13s}

The expression of Munc13 mRNA in the adult rat brain is shown in Fig 1.9 (Augustin et al., 1999a). Munc13-1, -2 and -3 showed neuron-specific expression in rat brain. Munc131 is expressed throughout many brain regions, with high expression in the olfactory bulb, striatum, cerebral cortex, CA regions and dentate gyrus of the hippocampus and cerebellum (Fig. 1.9A). Both Munc13-2 and Munc-13-3 show more restricted and seemingly complementary distributions, with Munc13-2 expressed mainly in rostral brain regions (olfactory bulb, cerebral cortex and CA regions of the hippocampus) (Fig. 1.9B) and Munc13-3 in caudal brain regions (cerebellum, brain stem, pons and dentate gyrus of hippocampus) (Fig. 1.9C). Protein expression of these three isoforms was found to largely match the mRNA expression pattern (Augustin et al., 1999a). In rat, the protein expression of all three isoforms in whole rat brains increased developmentally from birth and plateaued at post-natal day 15-22 (P15-P22) (Augustin et al., 1999a). Ultrastructurally, Munc13-1 was found to be localized to the active zone (Betz et al., 1998).

The expression of Baiap3 protein in the mouse brain is shown in Fig. 1.10. Baiap3 is highly expressed throughout the hypothalamus, in the central, medial and basomedial amygdaloid nuclei, paraventricular nucleus of the thalamus, septum, bed nucleus of the stria terminalis, the midbrain and the brain stem (Wojcik et al., 2013). Outside the nervous system Baiap3 RNA expression has been identified in syncytiotrophoblasts in mouse placenta, human breast epithelium and human prostate (Palmer et al., 2002). 


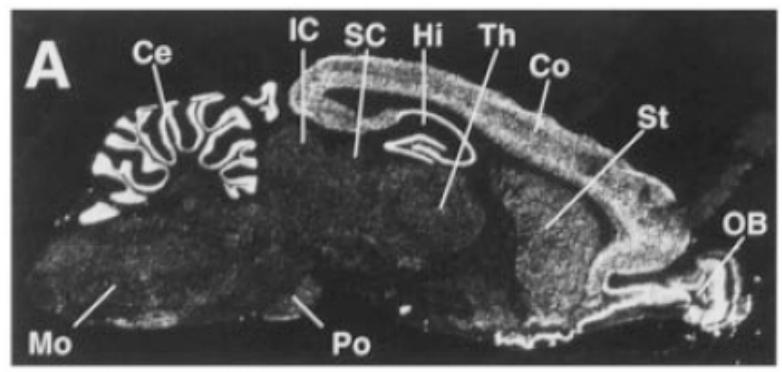

Munc 13-1

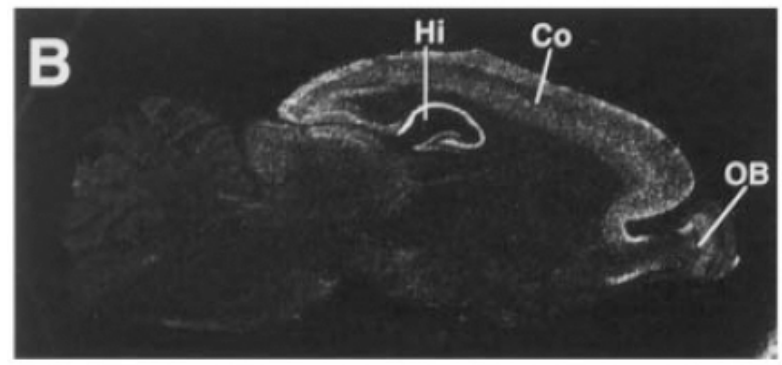

Munc 13-2

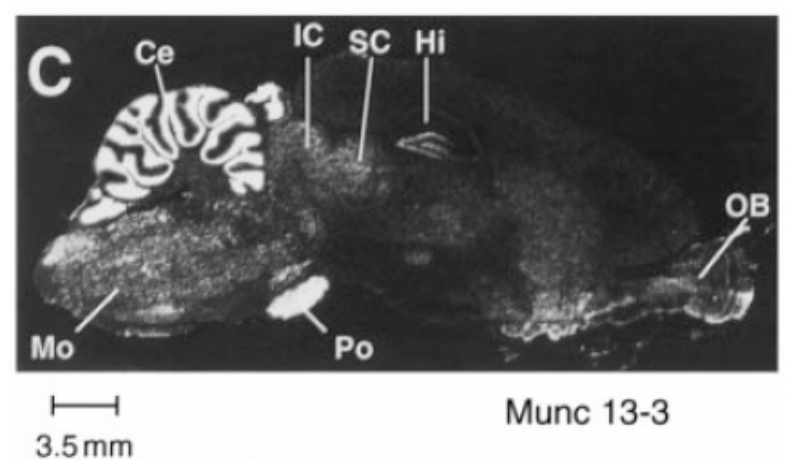

Fig. 1.9 In situ hybridization showing the expression pattern of Munc13-1, -2 and -3 mRNA in the rat brain (Augustin et al., 1999a). Ce, cerebellum; Co, cerebral cortex; Hi, hippocampus; IC, inferior colliculus; Mo, medulla oblongata; OB, olfactory bulb; Po, pons; $\mathrm{SC}$, superior colliculus; St, striatum; Th, thalamus. 


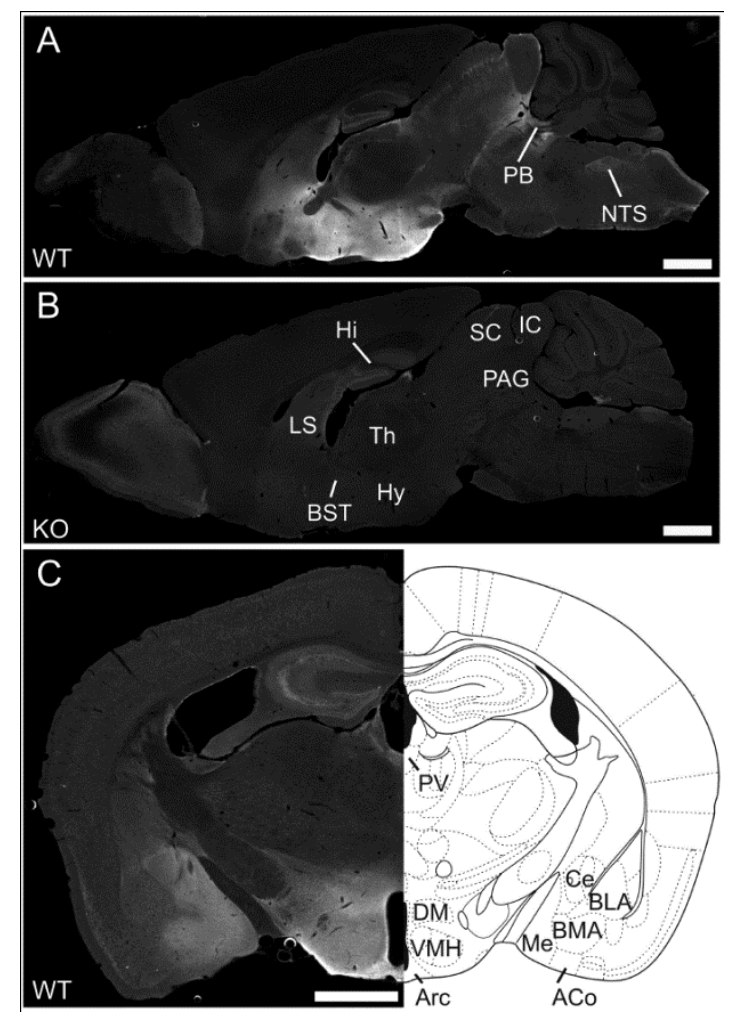

Fig. 1.10 Immunostaining showing the expression of Baiap3 in the adult mouse brain (Wojcik et al., 2013). PB, parabrachial nucleus; NTS, nucleus tractus solitarius; Hi, hippocampus; SC, superior colliculus; IC, inferior colliculus; PAG, periaqueductal gray; LS, lateral septum; Th, thalamus; Hy, hypothalamus; BST, bed nucleus of the stria terminalis; PV, paraventricular thalamic nucleus; DM, dorsomedial hypothalamic nucleus; VMH, ventromedial hypothalamic nucleus; Arc, arcuate nucleus; Ce, central amygdaloid nucleus; BLA, basolateral amygdaloid nucleus, anterior part; BMA, basomedial amygdaloid nucleus, anterior part; ME, medial amygdaloid nucleus; ACo, anterior corticalamygdaloid nucleus. Scale bars equal $1 \mathrm{~mm}$.

Munc13-4 protein was detected in rat spleen and lung, but not in the brain (Koch et al., 2000). Immunohistochemically, Munc13-4 was found to localise to globet cells of the bronchial epithelium and to alveolar type II cells in lung (Koch et al., 2000). Both cell types serve secretory functions. Munc13-4 was found to be expressed in a number of hematopoietic cells, including cytotoxic T lymphocytes (CTLs), where it was found to localise to cytolytic granules at the immunological synapse formed by cytotoxic T lymphocytes (Feldmann et al., 2003), and neutrophils, where it mediate the exocytosis of neutrophil granules (Pivot-Pajot et al., 2008).

The expression of major Munc13 isoforms was also observed in regions outside of the brain. Munc13-1, ubMunc13-2 and trace amounts of Munc13-4, but not bMunc13-2, Munc13-3 or Baiap3, were detected at the cholinergic neuromuscular junctions of the mouse 
diaphragm (Varoqueaux et al., 2005). In mouse retina, punctate staining of Munc13-1, bMunc13-2, ubMunc13-2 and Munc13-3 has been observed in the inner plexiform layer (IPL), where bipolar cell cells, amacrine cells and ganglion cells form conventional synapses (Cooper et al., 2012). In contrast, in the outer plexiform layer (OPL), where photoreceptor terminals form specialised ribbon synapses on bipolar cells and horizontal cells, only ubMunc13-2 staining was observed. Thus, ubMunc13-2 and bMunc13-2 were found to be selectively localised to ribbon synapses and conventional synapses, respectively (Cooper et al., 2012). In bovine chromaffin cells, low levels of Munc13-1, ubMunc13-2 and Munc13-3 have been detected, while bMunc13-2 was not expressed (Zikich et al., 2008). Munc13-1 is expressed in pancreatic islets of human, mouse and rat (Sheu et al., 2003).

\subsubsection{Role of Munc13 Isoforms in Priming and Docking}

The role of Munc13-1 and Munc13-2 in synaptic transmission has been studied extensively using autaptic cultures of mouse hippocampal neurons. Synaptic transmission at glutamatergic synapses and GABAergic synapses are either dependent on Munc13-1 or Munc13-2. In a given glutamatergic hippocampal neuron, $\approx 90 \%$ of synapses are dependent on Munc13-1 for transmission, while the remaining synapses are dependent on Munc13-2 (Rosenmund et al., 2002). GABAergic transmission is not affected in Munc13-1 KO mice (Augustin et al., 1999b). GABAergic hippocampal neurons from Munc13-2 KO mice showed normal evoked inhibitory post-synaptic current (IPSC) amplitudes, spontaneous miniature IPSC (mIPSC) frequencies and amplitudes, while Munc13-1/2 DKO hippocampal neurons are completely silent. Together with the absence of post-synaptic defects, these data demonstrate that endogenous expression of either Munc13-1 or Munc13-2 suffices to maintain normal GABAergic release in an inhibitory hippocampal neuron (Varoqueaux et al., 2002). Both evoked excitatory post-synaptic current (EPSC) amplitudes and evoked IPSC amplitudes can be rescued to near wild-type levels by the expression of either isoform in Munc13-1/2 DKO hippocampal neurons, indicating that these two isoforms can be employed for release irrespective of neurotransmitter type (Rosenmund et al., 2002). In the abovementioned Munc13 KO animals (Augustin et al., 1999b; Varoqueaux et al., 2002), as well as in unc-13 mutant C. elegans (Richmond et al., 1999) and dunc-13 null Drosophila (Aravamudan et al., 1999), where release was observed to be defective, no clear concomitant 
defects in docking were detected. Thus, Munc13 was proposed to act in a priming step downstream of docking to endow SVs with release competence.

More recent studies, where ultrastructural data were generated using high pressure freezing (HPF) in place of glutaraldehyde fixation, have reported different results. Glutaraldehyde is known to induce vesicle fusion (Smith and Reese, 1980), thus creating artefacts. In addition, HPF immobilises tissues in $\approx 10 \mathrm{~ms}$ (Siksou et al., 2009), while chemical fixation takes much longer to complete, allowing biological and degradation processes to continue for some time (Hammarlund et al., 2007). Using HPF, the number of SVs docked (exhibiting a "contact patch" with the plasma membrane) at the AZ in both cholinergic and GABAergic motor neurons in unc-13 mutant C. elegans was found to be reduced to $16 \%$ of wild-type, with accumulation of SVs in the peri-synaptic zone (Hammarlund et al., 2007). Spine synapses in hippocampal slices obtained from embryonic E18 Munc13-1/2 DKO mice and cultured for 5 weeks displayed almost a complete lack of vesicles in direct contact with the plasma membrane, while around half of the vesicles within $40 \mathrm{~nm}$ from the plasma membrane were docked in WT synapses (Fig. 1.11) (Siksou et al., 2009). The finding that Munc13 is not only required for priming but also for docking suggests that docking and priming may be one and the same molecular process (see section "Proposed Mechanisms of Munc13 Function"). 

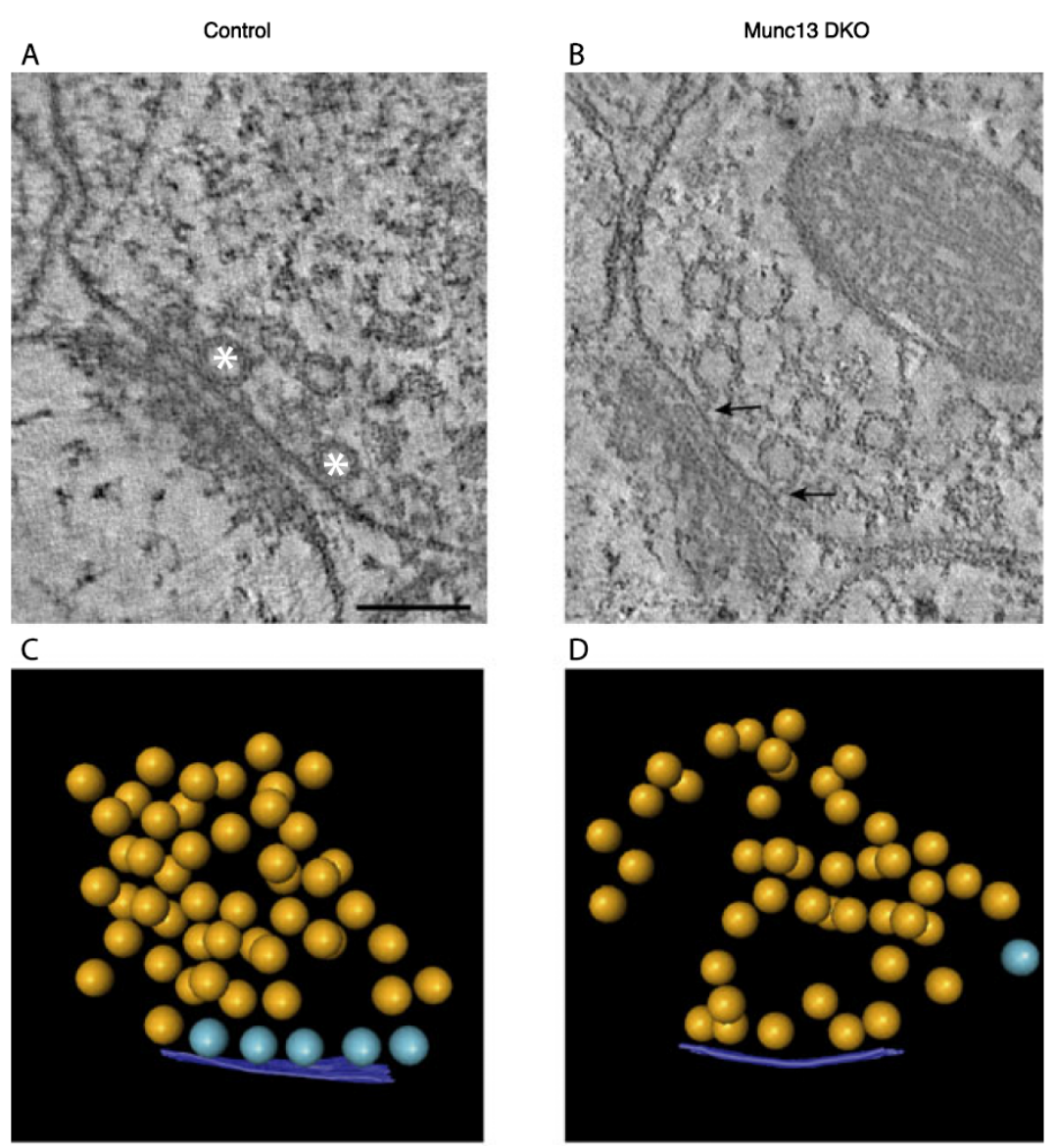

Fig. 1.11 Docking is drastically diminished in hippocampal cultured slices from Munc13-1/2 DKO mice (Siksou et al., 2009).

Munc13-4 is expressed in haematopoietic cells where it mediates priming of a range of exocytic vesicles. Mutations in human Munc13-4 cause familial hemophagocytic lymphohistiocytosis subtype 3 (FHL3), which is characterised by an overwhelming activation of $\mathrm{T}$ lymphocytes and macrophages, resulting in multi-systemic inflammation (Feldmann et al., 2003). Munc13-4 deficient cells were shown to be defective in the exocytosis of perforincontaining cytolytic granules from CTLs, although trafficking of lytic granules by microtubules to the contact site and morphological docking were not affected. Thus, Munc13-4 was proposed to underlie the priming of lytic granules in CTLs (Feldmann et al., 2003). Munc13-4 has been characterised as an effector of Rab27a to regulate dense core granule secretion of serotonin from platelets (Shirakawa et al., 2004) and lytic granule secretion in CTLs (Neeft et al., 2005). It was further shown to mediate secretory lysosome exocytosis in mast cells in a Doc2 $\alpha$-dependent manner (Higashio et al., 2008). Munc13-4 is also localised to endosomes in CTLs (Menager et al., 2007), where it probably functions together with Syntaxin-11 in fusing endosomes in preparation for granule maturation 
(Boswell et al., 2012). Munc13-4 accelerates SNARE-mediated liposome fusion and promotes trans-SNARE complex formation in a $\mathrm{Ca}^{2+}$-dependent manner. Both the Munc13-4 $\mathrm{C}_{2} \mathrm{~A}$ and $\mathrm{C}_{2} \mathrm{~B}$ domain (analogous to the $\mathrm{C}_{2} \mathrm{~B}$ and $\mathrm{C}_{2} \mathrm{C}$ domains in Munc13-1,-2 and -3 ) have been described as having specific $\mathrm{Ca}^{2+}$-dependent functions, namely t-SNARE protein binding and phosphatidylserine-containing membrane-binding. Munc13-4 with a mutated $\mathrm{C}_{2} \mathrm{~A}$ domain cannot accelerate liposome fusion as well as WT Munc13-4, however, a mutation in only the $\mathrm{C}_{2} \mathrm{~B}$ domain reduced acceleration to the same degree as the $\mathrm{C}_{2} \mathrm{AB}$ double mutant, indicating that the $\mathrm{C}_{2} \mathrm{~B}$ domain and its $\mathrm{Ca}^{2+}$-dependent membrane-binding function play a dominant role in the Munc13-4/Ca ${ }^{2+}$-dependent acceleration of SNAREmediated liposome fusion (Boswell et al., 2012).

Although brain-specific angiogenesis inhibitor-1 associated protein 3 (Baiap3) is known as a Munc13 isoform, its role in priming has not been described. Baiap3 was first identified as a binding partner of brain-specific angiogenesis inhibitor-1 (BAI-1) (Shiratsuchi et al., 1998), a G protein-coupled receptor enriched in the post-synaptic density that can activate both the Rho and extracellular-signal-regulated kinase (ERK) pathways (Stephenson et al., 2013). Baiap3 KO mice show an increase in anxiety that is more prominent in female mice, an altered development of tolerance to benzodiazepines in male mice, and an enhanced seizure propensity in both sexes (Wojcik et al., 2013). Action potential frequency in the hypothalamus, where Baiap3 is strongly expressed, is significantly increased in Baiap3 KO mice by $\approx 2.5$-fold compared to WT mice. In the human BAIAP3 gene two single-nucleotide polymorphism genotypes were identified that significantly correlate with the occurrence of benzodiazepine abuse in men, as well as with anxiety in women (Wojcik et al., 2013). Baiap3 has further been identified as a transcriptional target of EWS-WT1, a chimeric transcriptional factor, which causes desmoplastic small round cell tumor, an aggressive malignancy with origins from the serosal surfaces of the abdominal peritoneum (Palmer et al., 2002). However the molecular function of Baiap3 remains to be identified.

Munc13-1 (Ashery et al., 2000) and ubMunc13-2 (Zikich et al., 2008) have been shown to enhance priming of LDCVs in bovine chromaffin cells. Endogenous expression levels of Munc13-1, ubMunc13-2 and Munc13-3 were shown to be low (Zikich et al., 2008). Overexpression of Munc13-1 by Semliki Forest virus in bovine chromaffin cells caused a two- to three-fold increase in both the RRP and SRP, and $\mathrm{a} \approx 3.6$-fold increase in the sustained component of release. The ratio between the RRP and SRP sizes, as well as the time constants of the two pools was not altered by the overexpression. Treatment with PMA, 
a phorbol ester, caused membrane recruitment of EGFP-tagged Munc13-1 to the plasma membrane but did not further increase secretion. Expression of Munc13-1 with a mutation in the $\mathrm{C}_{1}$ domain that abolishes phorbol esters/diacylglycerol binding, was still able to significantly enhance release, although only to around $63 \%$ for the exocytotic burst and $45 \%$ for the sustained component of WT Munc13-1 expression (Ashery et al., 2000). Despite the large enhancement in exocytosis, no changes to the docking profile and the number of docked vesicles were observed, although the criteria for distinguishing docked vesicles were less stringent ( $\leq 200 \mathrm{~nm}$ within the plasma membrane) than in other studies. From these data, the authors concluded that overexpression of Munc13-1 affected only the priming of docked vesicles to the SRP, assuming a parallel pool model (Ashery et al., 2000). Overexpression of ubMunc13-2 in the same cell type caused an even more dramatic enhancement to release, amounting to $\mathrm{a} \approx 3.8$-fold increase in RRP size, $\approx 5$-fold increase in SRP size and $\approx 5$-fold increase in the sustained component (Zikich et al., 2008). The fast time constant was unaltered, but a longer slow time constant was observed in ubMunc13-2 overexpressing cells, which was attributed to the slowness of maturation of vesicles to reach release competence rather than to an SRP with intrinsically slow release. Overexpression of a calmodulin binding-deficient mutant was shown to affect the rate of vesicle replenishment at high $\left[\mathrm{Ca}^{2+}\right]$, but not at low $\left[\mathrm{Ca}^{2+}\right]$. Thus, it was proposed that although priming by ubMunc13-2 is $\mathrm{Ca}^{2+}$ dependent, it probably did not involve $\mathrm{Ca}^{2+} /$ calmodulin but other $\mathrm{Ca}^{2+}$-binding domains, such as the $\mathrm{C}_{2} \mathrm{~B}$ domain. In contrast, priming by ubMunc13-2 at high $\left[\mathrm{Ca}^{2+}\right]$ during ongoing activity required $\mathrm{Ca}^{2+} /$ calmodulin/ubMunc13-2 interactions (Zikich et al., 2008). Interestingly, in Munc13-1 overexpressing cells, the release triggered by a second flash at an inter-stimulation interval of 2 min has an amplitude similar to control cells (Ashery et al., 2000), while in ubMunc13-2 overexpressing cells, the second flash caused still more than 2fold enhancement as compared to control, indicating that ubMunc13-2 replenishes releasable pools faster than Munc13-1 (Zikich et al., 2008). Munc13-1 has been shown to be dispensable for LDCV release in chromaffin cells (Stevens et al., 2005). Recently, ubMunc13-2 expression has been demonstrated in PC12 cells and to translocate $\mathrm{Ca}^{2+}$ dependently to sites that are rich in phosphatidylinositol-4,5-bisphosphate $\left(\mathrm{PIP}_{2}\right)$, regions that are proposed as preferential sites for docking (Honigmann et al., 2013). Using a knockdown approach, ubMunc13-2 was shown to be required for DCV release from these cells (Kabachinski et al., 2013). 
Munc13-1 is expressed in pancreatic islets, which contain LDCVs for insulin release. Munc13-1 expression is reduced in type-2 diabetes non-obese Goto-Kakizaki and obese Zucker $f a / f a$ rats (Sheu et al., 2003). The absence of Munc13-1 in pancreatic $\beta$-cells from mice was found to abrogate the sustained component of insulin release after a step increase in $\left[\mathrm{Ca}^{2+}\right]$ by $\mathrm{Ca}^{2+}$ uncaging, without affecting the exocytotic burst (Kang et al., 2006). Replenishment of the RRP was also retarded in Munc13-1 KO $\beta$-cells. Maintenance of sustained release in WT mice were found to be partially dependent on endogenous levels of diacylglycerol, as the defect in sustained release was present but less severe in $\beta$-cells from $\mathrm{C}_{1}$ domain mutant mice than in Munc13-1 KO mice (Kang et al., 2006).

Munc13-1 was also found to play a role in regulating dense core vesicle (DCV) release in hippocampal neurons, where DCVs are released preferentially at synapses rather than at extra-synaptic sites (van de Bospoort et al., 2012). In Munc13-1/2 DKO neurons, DCV release at synapses was reduced by around $60 \%$, while release at extra-synaptic sites was reduced to a lesser extent. This reduction was largely attributed to the absence of Munc13-1. Overexpression of Munc13-1 in WT hippocampal neurons preferentially increased DCV exocytosis at extra-synaptic sites, without enhancing release at synaptic sites. It was concluded from these data that by localising to active zones, Munc13-1 contributes to maintaining the preference of release at synapses over extra-synaptic sites (van de Bospoort et al., 2012).

Unc-13 mutant C. elegans displayed reduced neuropeptide release from dorsal cord cholinergic motor neurons, with accumulation of DCVs in the ventral nerve cord (Sieburth et al., 2007). However, unc-13 was found to be dispensable in $\mathrm{Ba}^{2+}$-evoked release of peptide cargo from neurons in C. elegans (Speese et al., 2007). The discrepancy probably arose from differences in the preparations used (live animals and cultured neurons), as well as in the stimulation regimen (endogenous activity in live animals and $\mathrm{Ba}^{2+}$ stimulation). The requirement of unc-13 in DCV release from cell bodies of neurons, at least in C. elegans, remains unclear.

\subsubsection{Proposed Mechanisms of Munc13 Function}

Munc13 has been proposed to function through opening of syntaxin-1, thus removing the autoinhibitory interaction of the syntaxin-1 Habc domain and freeing the synataxin-1 
SNARE motif for SNARE complex formation. This model was proposed following the observation that expression of the open form of syntaxin (Dulubova et al., 1999) in the neuromuscular junction can completely rescue evoked EPSC amplitude of unc-13 mutant $C$. elegans, although locomotor activity in these mutants was still largely hampered as compared to WT worms, and the frequency of endogenous synaptic events in the presence of $\mathrm{Ca}^{2+}$ was rescued to only $\approx 20 \%$ of WT levels (Richmond et al., 1999). Subsequent studies reported incongruent findings in the same preparation, showing that despite the restoration of a primed pool of vesicles to $\approx 70 \%$ of WT levels as measured by the application of hyperosmotic sucrose, the amplitude of evoked release and frequency of endogenous synaptic events were significantly reduced in unc-13/open-syntaxin double mutant worms compared with WT levels (McEwen et al., 2006). Using HPF techniques for EM, the open-syntaxin allele was found to fully rescue the reduction in docking at the $\mathrm{AZ}$ in unc-13 mutant worms, but the amplitude of evoked release was still only $\approx 38 \%$ and endogenous fusion events $\approx 26 \%$ that of WT (Fig. 1.12). Further, release kinetics in these double mutant worms were slower and release was more asynchronous than in WT worms (Hammarlund et al., 2007). The fact that open syntaxin is able to almost fully rescue sucrose-evoked release and to fully rescue docking, but only partially rescue evoked release amplitudes, supports a view that unc-13 may serve dual roles in two sequential steps. First, docking requires the opening of syntaxin by $u n c-13$. Second, unc-13 is required in a priming step after docking, which may also involve unc-13/syntaxin interaction, e.g. mediated by the $u n c-13 \mathrm{C}_{2} \mathrm{C}$ domain (Madison et al., 2005; McEwen et al., 2006). 
A Vesicle docking in unc-13 open syntaxin
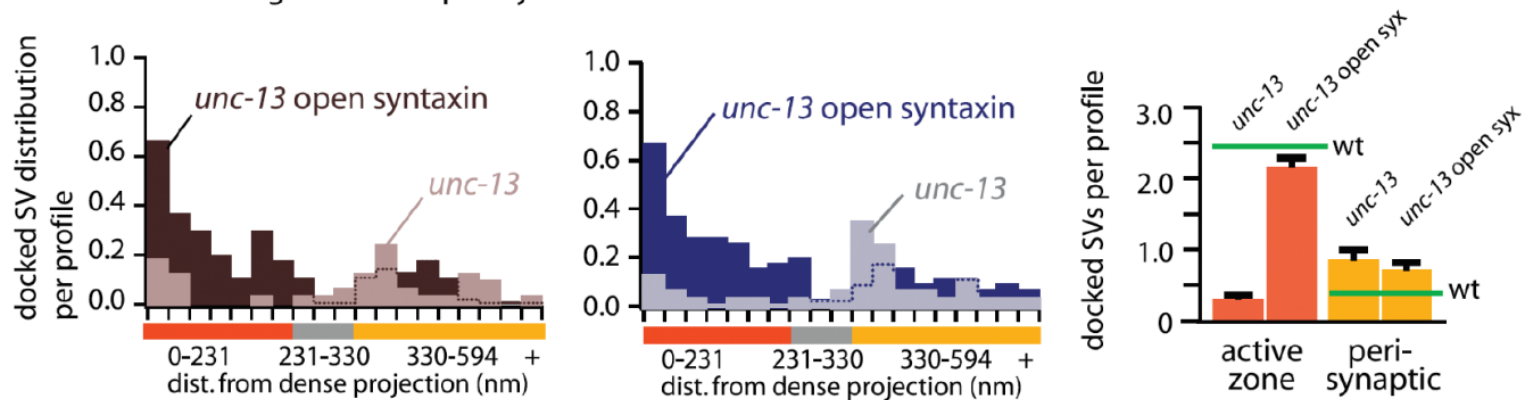

B
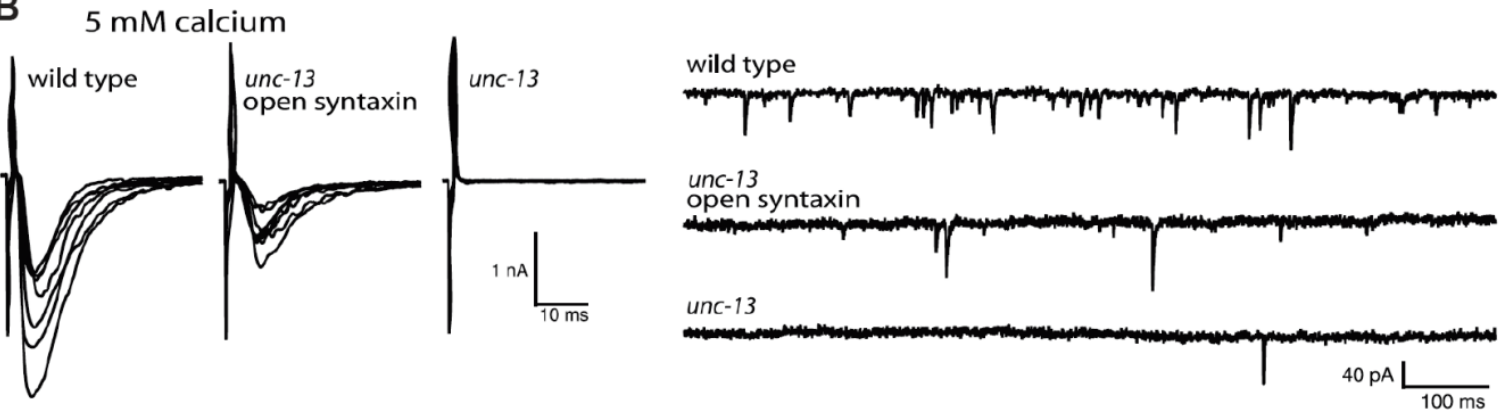

Fig. 1.12 Full rescue of docking but incomplete rescue of evoked EPSCs by crossing unc13 mutant $C$. elegans with a mutant expressing open syntaxin (modified from (Hammarlund et al., 2007)).

The mechanism of how Munc13s open syntaxin is unclear. It has been proposed that the MUN domain extracts the SNARE motif from the syntaxin-1/Munc18 closed complex through weak interactions to promote SNARE complex formation. Indeed, a mutation disrupting the MUN/syntaxin SNARE motif interactions was found to retard the MUNmediated acceleration of SNARE complex formation by a Munc18 bound form of syntaxin-1 (Ma et al., 2011). The MUN domain was also found to bind to membrane-anchored syntaxin1/SNAP-25 heterodimers and membrane-anchored SNARE complexes, and had been suggested to provide a platform on which syntaxin-1/SNAP-25 heterodimers can be assembled (Guan et al., 2008).

Munc13s form a tripartite complex with Rab3-interacting molecules (RIMs) and Rab3, a family of small synaptic vesicular GTPases (Dulubova et al., 2005). RIM activates Munc13 function by resolving Munc13 homodimers formed via mutual $\mathrm{C}_{2} \mathrm{~A}$ domain interaction (Deng et al., 2011) by binding to regions around and including the $\mathrm{C}_{2} \mathrm{~A}$ domain (residue 3-209 of Munc13-1) with its zinc-finger (ZF) domain (Dulubova et al., 2005). Since RIM was found to tether $\mathrm{Ca}^{2+}$ channels to pre-synaptic active zones with its PDZ domain and its interaction with RIM-binding protein (RIM-BP) (Kaeser et al., 2011), a multi-protein 
complex consisting of Munc13, RIM, Rab3 and RIM may tether vesicles close to $\mathrm{Ca}^{2+}$ channels and co-localize vesicles with the priming machinery to enable fast, synchronous release (Kaeser, 2011).

\subsubsection{Short-term Synaptic Plasticity Characteristics of Munc13 Isoforms}

Although both glutamatergic and GABAergic hippocampal synapses can utilize either Munc13-1 or Munc13-2 to mediate vesicular neurotransmitter exocytosis (Rosenmund et al., 2002), the two Munc13 isoforms show vastly different short-term synaptic plasticity characteristics. Hippocampal glutamatergic neurons from Munc13-1 KO mice, where $90 \%$ of synapses are dependent on Munc13-1, showed an initial depression but delayed facilitation of $\approx 170 \%$ at steady state not seen in WT when stimulated at $10 \mathrm{~Hz}$. The facilitation observed was attributed to the remaining $10 \%$ of synapses that used Munc13-2 as their priming factor. Rescue of Munc13-1/2 DKO neurons with viral expression of the two isoforms showed that release driven by Munc13-1 showed depression similar to the response in WT cells, while release driven by Munc13-2 showed facilitation similar to the response in Munc13-1 KO neurons under $10 \mathrm{~Hz}$ train stimulation. The inherent difference of the two isoforms was further exemplified by the higher degree of short-term augmentation seen in Munc13-2expressing as compared to Munc13-1-overexpressing cells, following a $10 \mathrm{~Hz}$ high frequency train for 5 s (Junge et al., 2004; Rosenmund et al., 2002). Rescue of Munc13-1/2 DKO hippocampal neurons with both bMunc13-2 and Munc13-3 showed short-term frequency facilitation, but the augmentation seen following a $10 \mathrm{~Hz}$ train stimulation in cells rescued with Munc13-3 was not as strong as that in bMunc13-3 (Lipstein et al., 2012). These studies illustrate the inherent differences of Munc13 isoforms in driving refilling of the RRP and synaptic vesicle priming.

The augmentation characteristic of ubMunc13-2 was thought to be regulated by the $\mathrm{Ca}^{2+}$-binding $\mathrm{C}_{2} \mathrm{~B}$ domain. $\mathrm{A} \quad \mathrm{C}_{2} \mathrm{~B}$ mutant that failed to bind $\mathrm{Ca}^{2+}$ abolished phosphatidylinositol phosphate (PIP) and $\mathrm{PIP}_{2}$ binding of ubMunc13-2 and showed a loss of augmentation after a $10 \mathrm{~Hz}$ high frequency train, without affecting the size of the RRP, amplitude of evoked EPSC and $\mathrm{Ca}^{2+}$-dependence of evoked EPSC amplitude (Shin et al., 2010). Based on these findings the authors proposed a model in which high synaptic activity increases intracellular $\left[\mathrm{Ca}^{2+}\right]$ as well as membrane concentrations of PIP and $\mathrm{PIP}_{2}$. Subsequently, $\mathrm{Ca}^{2+}$-dependent phospholipid-binding of ubMunc13-2 is activated and 
recruitment of the protein to the membrane is increased, resulting in augmentation (Shin et al., 2010).

\subsection{Role of CAPS in Priming and Docking}

$\mathrm{Ca}^{2+}$-dependent activator protein for secretion (CAPS) proteins have been shown to be involved in the release of both DCVs (Liu et al., 2008; Speese et al., 2007; Zhou et al., 2007) and synaptic vesicles (Jockusch et al., 2007). Two isoforms for CAPS exist, namely CAPS-1 and CAPS-2. In the rat, CAPS-1 expression is largely restricted to the brain and neuroendocrine tissues such as the adrenal gland and the pancreas, whereas CAPS-2 is expressed in brain, liver, testis and lung (Speidel et al., 2003). While CAPS-1 is widely expressed throughout the brain and CAPS-2 shows expression restricted to selected brain regions, immunostaining showed that CAPS-1 and CAPS-2 expression largely complements each other (Speidel et al., 2003). In brain, CAPS-1 is developmentally upregulated from E14 until P20. By comparison, CAPS-2 expression is more stable, with slightly higher expression in the embryonic brain than that after birth. Ultrastructurally, CAPS-1 was shown to colocalise with LDCVs and also to preferentially localise to asymmetric/glutamatergic axon terminals over symmetric/GABAergic terminals (Speidel et al., 2003). The orthologue of CAPS in C. elegans, unc-31, was shown to be involved in the release of neuropeptidecontaining DCVs from ventral cord cholinergic motor neurons (Sieburth et al., 2007), in $\mathrm{Ba}^{2+}$-evoked release (Speese et al., 2007) and in $\mathrm{Ca}^{2+}$-evoked release (Zhou et al., 2007) of DCV cargo from cultured C. elegans neurons. PMA binding to Munc13s/unc-13 not only potentiates SV release, but also DCV release in C. elegans ALA neurons (Zhou et al., 2007). Strikingly, the potentiation did not manifest in both unc-31 and unc-13 mutant neurons, suggesting that CAPS/unc-31 may act downstream of Munc13/unc-13 in DCV exocytosis (Zhou et al., 2007). CAPS was found to be required for docking of DCVs to the plasma membrane in C. elegans ventral cord motor neurons. The docking deficiency in unc-31 mutant worms can be rescued by overexpression of open syntaxin (Hammarlund et al., 2008).

Similar to their expression in the brain, CAPS-1 and CAPS-2 display developmental upregulation and downregulation in the adrenal gland, respectively (Speidel et al., 2005). In embryonic CAPS-1 KO chromaffin cells, the spike frequency detected amperometrically during high $\mathrm{K}^{+}$stimulation was reduced, while no defects in capacitance increase following step increases in $\left[\mathrm{Ca}^{2+}\right]$ or trains of depolarisation was observed. It was concluded from this 
finding that CAPS-1 is associated with the loading of catecholamines to LDCVs (Speidel et al., 2005). In flash photolysis of caged $\mathrm{Ca}^{2+}$ experiments in CAPS-1/2 DKO chromaffin cells, the RRP was reduced by $\approx 50 \%$, while the sustained component was nearly absent (Liu et al., 2008). No docking defect accompanied the reduction of LDCV exocytosis. CAPS was thus established as a priming factor for LDCV in chromaffin cells (Liu et al., 2008). CAPS have been shown to accelerate the fusion of liposomes with t-SNARE acceptor complexes and synaptobrevin-2 in a PIP $_{2}$-dependent manner (James et al., 2009). Interestingly, expression of open syntaxin rescued the defective RRP size in CAPS-1/2 DKO chromaffin cells, but not the sustained component, and Munc13-1 overexpression cannot rescue the defective RRP size. This latter result supports that Munc13 may act upstream of CAPS (Liu et al., 2010).

While CAPS proteins are essential in LDCV exocytosis, their role in SV exocytosis appears may be more species specific. Unc-31 was shown to be dispensable in $\mathrm{Ca}^{2+}$ dependent SV release from cultured C. elegans neurons (Speese et al., 2007; Zhou et al., 2007). In contrast, CAPS appears to be required for SV release in hippocampal neurons from mice. In hippocampal neurons from CAPS-1/2 DKO mice, RRP size, evoked EPSC amplitude, mEPSC amplitude and frequency was significantly reduced as compared with CAPS-2 single KO cells, demonstrating that CAPS-1 is important in SV release (Jockusch et al., 2007). No docking defects of SVs in these neurons were observed, consistent with a priming role for SV. The RRP size and evoked EPSC amplitudes of CAPS-1/2 DKO neurons can be restored after $40 \mathrm{~Hz}$ stimulation which causes an increase in intracellular $\left[\mathrm{Ca}^{2+}\right]$ (Jockusch et al., 2007). Overexpression of Munc13-1 in CAPS-1 DKO cells and overexpression of CAPS-1 in Munc13-1/2 DKO cells both fail to cross rescue the release deficits, which is suggestive of Munc13 and CAPS acting in an interdependent manner in the same pathway in the regulation of vesicle exocytosis (Jockusch et al., 2007; Kabachinski et al., 2013).

\subsection{Scope of the Thesis}

As discussed above, several lines of evidence suggest that Munc13s as well as CAPS proteins regulate exocytosis of SVs and LDCVs. However, a deficit in LDCV exocytosis in Munc13-deficient chromaffin cells had not been demonstrated. I therefore investigated LDCV exocytosis in chromaffin cells lacking the Munc13 isoforms Munc13-1, Munc13-2, 
Munc13-3 or Baiap3. This study aims at producing a comprehensive analysis of the contribution of individual Munc13 isoforms to LDCV release in a well-characterised model system, the chromaffin cell, to shed light on a more unified picture of the regulated exocytosis of vesicles. 


\section{Materials and Methods}

\subsection{Transgenic Mouse Lines and Genotyping}

Munc13-1 knockout (KO) (Augustin et al., 1999b), Munc13-1/2 double KO (DKO) (Varoqueaux et al., 2002), Munc13-3 KO (Augustin et al., 2001), Baiap3 KO (Wojcik et al., 2013), Munc13-1-EYFP knockin (KI) (Kalla et al., 2006), Munc13-2-EYFP KI and Munc133-EGFP-KI (Cooper et al., 2012) mice have been described before. Tail DNA was obtained according to manufacturer's instructions using the nexttec ${ }^{\mathrm{TM}} 1$-step DNA isolation system (nexttec ${ }^{\mathrm{TM}}$ Biotechnologie $\mathrm{GmbH}$ ). Genotyping was performed for each experiment by a standard polymerase chain reaction (PCR) (Maniatis, 1982) and modified as described below using RedTaq polymerase (Sigma). PCR reactions were prepared by mixing on ice $2 \mu 1$ of 10x buffer, $0.5 \mathrm{mM}$ deoxyribonucleotide triphosphates (dNTPs), $5 \mu \mathrm{M}$ of each primer, $1 \mu \mathrm{l}$ of RedTaq polymerase, $1 \mu \mathrm{l}$ of template DNA and sufficient $\mathrm{H}_{2} \mathrm{O}$ to make up a final reaction volume of $20 \mu \mathrm{l}$. The primer sequences used for each line, the annealing temperature and the number of cycles used are shown in Table 2.1. In a typical reaction programme, reactions were incubated at $94^{\circ} \mathrm{C}$ for $3 \mathrm{~min}$ for initial DNA denaturation, followed by 3 -step cycles consisting of denaturation at $94^{\circ} \mathrm{C}$ for $3 \mathrm{~min}$, annealing at the suitable annealing temperature $\left(\mathrm{T}_{\mathrm{m}}\right.$, see Table 2.1) for $30 \mathrm{~s}$, and extension at $72^{\circ} \mathrm{C}$ for around $1 \mathrm{~min}$. The number of cycles ranged from 30-35 (Table 2.1). The final step is a $72^{\circ} \mathrm{C}$ incubation for $7 \mathrm{~min}$ for final extension. Reactions were kept at $10^{\circ} \mathrm{C}$ before analysis on agarose gels. 


\begin{tabular}{|c|c|c|c|c|c|}
\hline $\begin{array}{c}\text { Mouse } \\
\text { line }\end{array}$ & Allele & Primer sequence & $\begin{array}{l}\text { Fragment } \\
\text { length (bp) }\end{array}$ & $\begin{array}{c}\mathbf{T}_{\mathrm{m}} \\
\left({ }^{\circ} \mathbf{C}\right)\end{array}$ & $\begin{array}{c}\text { Cycle } \\
\text { no. }\end{array}$ \\
\hline \multirow{4}{*}{$\begin{array}{l}\text { Munc13-1 } \\
\text { KO }\end{array}$} & \multirow{2}{*}{ WT } & 5'-CTTACCCATCTGAGAGCCGGAATTCCA-3' & \multirow{2}{*}{250} & \multirow{4}{*}{60} & \multirow{4}{*}{30} \\
\hline & & 5'-CTCCGAGGGGAATGCGCTTCCGTTTCCTG-3' & & & \\
\hline & \multirow{2}{*}{ KO } & 5'- CTTACCCATCTGAGAGCCGGAATTCCA-3' & \multirow{2}{*}{230} & & \\
\hline & & 5'-GAGCGCGCGCGGCGGAGTTGTTGAC-3' & & & \\
\hline \multirow{4}{*}{$\begin{array}{l}\text { Munc13-2 } \\
\text { KO }\end{array}$} & \multirow{2}{*}{ WT } & 5'-CTCCACTGCCCCCTTTTACTGT-3' & 322 & \multirow{4}{*}{60} & \multirow{4}{*}{30} \\
\hline & & 5'-TCAAGGGACTGTTCTAGCAATGTT-3' & $32 L$ & & \\
\hline & \multirow{2}{*}{$\mathrm{KO}$} & 5'-СТCCACTGCCCCCTTTTACTGT-3' & 40 & & \\
\hline & & 5'-GAGCGCGCGCGGCGGAGTTGTTGAC-3' & 349 & & \\
\hline \multirow{4}{*}{$\begin{array}{l}\text { Munc13-3 } \\
\text { KO }\end{array}$} & \multirow{2}{*}{ WT } & 5'-GGCTAGGAAGCAGGTAGTGATGGCTG -3' & 202 & \multirow{4}{*}{62} & \multirow{4}{*}{35} \\
\hline & & 5'-GCTTAACTGGAACTCACTGGATGTCAGAG -3' & 393 & & \\
\hline & \multirow[b]{2}{*}{$\mathrm{KO}$} & 5'-GACGAGTTCTTCTGAGGGGATCGGC -3' & \multirow[b]{2}{*}{407} & & \\
\hline & & $\begin{array}{l}\text { 5'-TACATTAGAGATGATAATTATCACACCCCAAAG } \\
-3 \text { ' }\end{array}$ & & & \\
\hline \multirow{4}{*}{$\begin{array}{l}\text { Baiap3 } \\
\text { KO }\end{array}$} & \multirow{2}{*}{ WT } & 5'-CCAGAAATCCGCAGGCAGTCGTCA -3' & 546 & \multirow{4}{*}{63} & \multirow{4}{*}{34} \\
\hline & & 5'-CAAGGCAACCACCAGCCGCATCTA -3' & 540 & & \\
\hline & \multirow{2}{*}{ KO } & 5'-CCAGAAATCCGCAGGCAGTCGTCA -3' & 701 & & \\
\hline & & 5'-GAACACGGCGGCATCAGAGCAG -3' & 101 & & \\
\hline \multirow{4}{*}{$\begin{array}{c}\text { Munc13- } \\
\text { 1-EYFP } \\
\text { KI }\end{array}$} & \multirow{2}{*}{ WT } & 5'-FAM-AGCGCAGCAACGATGAGGTG-3' & & \multirow{4}{*}{64} & \multirow{4}{*}{30} \\
\hline & & 5'-GAGAGACGGTGGCCTACGGA-3' & 162 & & \\
\hline & \multirow{2}{*}{ KI } & 5'-CATGGTCCTGCTGGAGTTCGTG-3' & 235 & & \\
\hline & & 5'-GAGAGACGGTGGCCTACGGA-3' & 225 & & \\
\hline \multirow{4}{*}{$\begin{array}{l}\text { Munc13- } \\
\text { 2-EYFP } \\
\text { KI }\end{array}$} & \multirow{2}{*}{ WT } & 5'-GATGAGACAGGCATGACCAT-3' & 190 & \multirow{4}{*}{61} & \multirow{4}{*}{30} \\
\hline & & 5'-ACAGCTAACTCTCCCTGACTGA-3' & 190 & & \\
\hline & KI & 5'-CATGGTCCTGCTGGAGTTCGTG-3' & 220 & & \\
\hline & KI & 5'-ACAGCTAACTCTCCCTGACTGA-3' & 220 & & \\
\hline & & 5'-TCTCTCAGAGGACCAGCGA-3' & 546 & & \\
\hline Munc13- & W1 & 5'-TGGCACTTCATGGAACATTTAT-3' & 346 & 58 & 30 \\
\hline $\begin{array}{l}\text { S-LU } \\
\text { KI }\end{array}$ & $\mathrm{KI}$ & 5'-CATGGTCCTGCTGGAGTTCGTG-3' & & 30 & 30 \\
\hline & $\Lambda I$ & 5'-TGGCACTTCATGGAACATTTAT-3' & 701 & & \\
\hline
\end{tabular}

Table 2.1. Primer sequences used for genotyping of transgenic mouse lines.

The presence and sizes of the PCR products were analysed with $2 \%$ agarose gels in a Tris-borate buffer (0.09 M Tris-borate, $0.002 \mathrm{M}$ ethylenediaminetetraacetic acid (EDTA), pH 8.0) according to previously established protocols (Maniatis, 1982). For visualising the DNA under UV illumination at $312 \mathrm{~nm}$, ethidium bromide was added to the gel at a final concentration of $500 \mathrm{ng} / \mathrm{ml}$. Gels were documented with a gel documentation system (Intas). 


\subsection{Western Blotting}

Western blots were performed according to standard procedures (Maniatis, 1982) with modifications as stated below. Perinatal adrenal gland homogenates were made by pooling adrenal glands from E18 (Munc13-1 KO, Munc13-1/2 DKO and control littermates) or P0 animals (Munc13-2 KO, Munc13-3 KO, Baiap3 KO, Munc13-1-EYFP KI, Munc13-2-EYFP KI, Munc13-3-EGFP KI and control littermates) with the same genotype using a Potter S homogeniser in an ice-cold homogenisation buffer (320 mM glucose, $20 \mathrm{mM}$ HEPES, $2 \mathrm{mM}$ EDTA, pH 7.4; $0.5 \mu \mathrm{g} / \mathrm{ml}$ leupeptin, $1 \mu \mathrm{g} / \mathrm{ml}$ aprotinin and $0.1 \mathrm{mM}$ phenylmethylsulfonyl fluoride (PMSF) was added immediately prior to use) at $900 \mathrm{rpm}$ for around $45 \mathrm{~s}$ for each sample at $4^{\circ} \mathrm{C}$. A custom-made plunger suitable for homogenisation in an eppendorf tube was used. Sufficient buffer was used to result in a concentration of a few $\mu \mathrm{g} / \mu \mathrm{l}(\approx 250 \mu \mathrm{l}$ per 25 pairs of perinatal adrenal glands). Whole brain samples from E18 or P0 and from adult animals were homogenised using plungers and cylinders of appropriate sizes and $1 \mathrm{ml}$ and 10 $\mathrm{ml}$ buffer per brain, respectively. After homogenization, brain samples were centrifuged for $10 \mathrm{~min}$ at $1000 \mathrm{~g}$ at $4^{\circ} \mathrm{C}$ to pellet the nuclear fraction (Graham, 2002) and the resultant postnuclear supernatant was used. Proteins were kept on ice whenever possible during the entire homogenisation procedure. Protein samples were denatured in Laemmli buffer $(30 \mathrm{~g} / \mathrm{L}$ sodium dodecyl sulphate (SDS), $41.7 \mathrm{mM}$ Tris pH 6.8, $1 \mathrm{mM}$ EDTA, $100 \mathrm{~g} / \mathrm{L}$ sucrose, 50 $\mathrm{mM}$ dithiothrietol (DTT), $0.074 \%$ bromophenol blue) by heating at $96^{\circ} \mathrm{C}$ for $3 \mathrm{~min}$. After denaturation, protein samples were immediately placed on ice. Samples containing $20 \mu \mathrm{g}$ protein were separated on a $7.5 \%$ gel for SDS-polyacrylaminde gel electrophoresis (SDSPAGE). The buffer for the separating gel contained 1.5 M Tris and 0.4\% SDS (pH 8.8). For

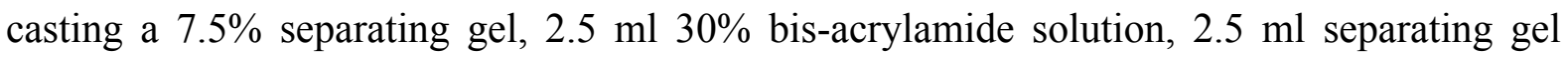
buffer, $4.8 \mathrm{ml} \mathrm{H} \mathrm{H}_{2} \mathrm{O}, 62.5 \mu \mathrm{l} 10 \%$ ammonium persulphate and $12.5 \mu \mathrm{l} N, N, N^{\prime}, N^{\prime}-$ Tetramethylethylenediamine (TEMED) were mixed. The stacking gel buffer contained $0.5 \mathrm{M}$ Tris and $0.4 \%$ SDS (pH 6.8). To cast the stacking gel, $1.25 \mathrm{ml} \mathrm{30 \%} \mathrm{bis-acrylamide} \mathrm{solution,}$ $2.5 \mathrm{ml}$ stacking gel buffer, $6.05 \mathrm{ml} \mathrm{H}_{2} \mathrm{O}, 62.5 \mu \mathrm{l}$ 10\% ammonium persulphate and $12.5 \mu \mathrm{l}$ TEMED was mixed. The SDS-PAGE running buffer contained $25 \mathrm{mM}$ Tris, $250 \mathrm{mM}$ glycine $\mathrm{pH} 8.3$ and $0.1 \%$ SDS. After SDS-PAGE, proteins were transferred onto nitrocellulose membranes (Millipore) in a transfer buffer (192 mM glycine, $25 \mathrm{mM}$ Tris, 20\% methanol) at 20-25 V for $16 \mathrm{~h}$ at $4^{\circ} \mathrm{C}$. A $10 \mathrm{x}$ TBS buffer (1.37M NaCl, $200 \mathrm{mM}$ Tris-HCl, $\mathrm{pH}$ 7.6) was used as a stock solution to prepare the membrane blocking buffer, antibody incubation buffer and washing buffer as described below. Following transfer, membranes were rinsed once 
with $1 \mathrm{x}$ TBS with $0.1 \%$ Tween-20 and non-specific epitopes blocked with a solution which contained 5\% goat serum, 5\% dry milk powder, 1x TBS buffer and $0.1 \%$ Tween-20 at room temperature (RT) for $30 \mathrm{~min}$. Then, primary antibody incubations with the relevant primary antibody diluted in the blocking buffer was performed at $4^{\circ} \mathrm{C}$ overnight. An exception was made when blotting for Munc13-4, in which 2\% donkey serum was used in place of $5 \%$ goat serum in the blocking step and in antibody incubations, because the Munc13-4 antibody was raised in goat. Primary antibodies used were as follows with the indicated dilutions. Munc131 antibody (Cat\#126 103, 1:500) and chromogranin A antibody (affinity purified form of Cat\#259 002, 1:8000) were from Synaptic Systems. Antibodies against ubMunc13-2 (affinity purified, batch \#52, 1:2000), bMunc13-2 (affinity purified, batch \#50, 1:1000), Munc13-3 (affinity purified, batch \#48, 1:500) (Cooper et al., 2012; Varoqueaux et al., 2005) and Baiap3 ("SA5990" or "SA5991”, 1:1000) (Wojcik et al., 2013) were raised in the lab previously. Munc13-4 antibody (1:250) was from Novus Biologicals (Cat\#NB100-41385). Green fluorescent protein (GFP) antibody (1:500) was from Roche (Cat\#11814460001). Valosin-containing protein (VCP) antibody (1:1000) was from BD Transduction Laboratories (Cat\#612182). Alternatively, an antibody (1:2000), which was a gift from Prof. Thomas Südhof, was used for VCP detection. Glyceraldehyde 3-phosphate dehydrogenase (GAPDH) antibody (1:25000) was from Abcam (Cat\#ab8245). After primary antibody incubation, membranes were rinsed once and washed with gentle shaking in a washing buffer (1x TBS, 5\% dry milk powder and $0.1 \%$ Tween-20) 3 times, with the first two washes lasting for 15 min and the last lasting for $5 \mathrm{~min}$. Secondary antibodies conjugated with horseradish peroxidase were used at dilutions between 1:2000 and 1:20000. This was followed by rinsing once and washing for 3 times with the washing buffer as above. Membranes were then rinsed once and washed for $5 \mathrm{~min}$ in $1 \mathrm{x}$ TBS buffer with $0.1 \%$ Tween-20. After a final wash step by rinsing once and washing for 5 min with 1x TBS buffer, membranes were incubated with chemiluminescence reagents in the dark. For Munc13-3, SuperSignal West Dura Chemiluminescent Substrate (Thermo Scientific, Cat\#64705) was used for detection. For other isoforms, standard enhanced chemiluminescent (ECL) reagents were used (Amersham, Cat\#RPM2134). 


\subsection{Chromaffin Cell Culture}

Cultures of chromaffin cells from perinatal animals were prepared according to published protocols (Sorensen et al., 2003). WT littermate animals were used as control, except for the experiment involving Munc13-1/2 DKO animals, where animals of different genotypes were prepared depending on their availability in a given litter. Adrenal glands were excised from E18 embryos (for experiments involving Munc13-1 KO and Munc13-1/2 DKO animals) or P0 animals (all other experiments) and placed in ice-cold Locke's solution (154mM NaCl, $5.58 \mathrm{mM} \mathrm{KCl}, 0.84 \mathrm{mM} \mathrm{NaH}_{2} \mathrm{PO}_{4}, 2.14 \mathrm{mM} \mathrm{Na}_{2} \mathrm{HPO}_{4}$ and $10 \mathrm{mM}$ glucose). From this point onwards, the culture procedure was performed in a sterile environment. Glands were incubated in $300 \mu$ papain solution $(200 \mathrm{mg} / \mathrm{L} \mathrm{L}$-cysteine, $1 \mathrm{mM} \mathrm{CaCl}, 0.5 \mathrm{mM}$ EDTA, 20U papain/ml (Worthington, Cat\#LS003127)) in Dulbecco's Modified Eagle Medium, DMEM (Gibco), bubbled in advance for $15 \mathrm{~min}$ with carbogen) at $37^{\circ} \mathrm{C}$ with gentle shaking $(450 \mathrm{rpm})$ for $45 \mathrm{~min}$, followed by an inactivation procedure in which an equal volume of a stop solution (10\% fetal bovine serum (Gibco, Cat\#10500-064), $2.5 \mathrm{~g} / \mathrm{L}$ trypsin inhibitor and $2.5 \mathrm{~g} / \mathrm{L}$ albumin in $\operatorname{DMEM}($ Gibco)) was added and glands were incubated for 5 $\min$ at $37^{\circ} \mathrm{C}$. Without disturbing the glands, the mixture was replaced with $160 \mu \mathrm{DMEM}$ (Linaris, Cat\#GMF2143YK) supplemented with 1\% insulin-transferrin-selenium-X (ITS-X) supplement (Gibco, Cat\#51500-056) and 200 U/L penicillin-streptomycin (Gibco, Cat\#15070-063). Glands were gently triturated with a $200 \mu$ l pipette tip until a cell suspension was obtained. $50 \mu \mathrm{l}$ of the cell suspension was placed onto the centre of glass coverslips in a 6-well plate. Cells were placed in a humidified incubator at $37^{\circ} \mathrm{C}$ and $8 \% \mathrm{CO}_{2}$ and allowed to settle onto the coverslips for $30 \mathrm{~min}$ and $2 \mathrm{ml}$ supplemented DMEM (supplemented with 1\% ITS-X and $200 \mathrm{U} / \mathrm{L}$ penicillin-streptomycin, as mentioned above) were then added into each well. Cells were used on DIV2 and/or DIV3.

\subsection{Virus Preparation and Infection}

Rescue experiments of Munc13-1/2 DKO cells with ubMunc13-2 and Baiap3, and overexpression of Baiap3 in WT cells by Semliki Forest viral expression were performed as follows. A construct containing the ubMunc13-2 open reading frame fused with an enhanced green fluorescent protein (EGFP) sequence was cloned into the viral vector pSFV-1 for the production of Semliki Forest virus (Ashery et al., 1999). A pSFV-1 construct for the expression of Baiap3 was generated with an internal ribosomal entry site upstream of the 
EGFP sequence. Since the efficiency for virus infection varied among different viruses and among different batches of the same virus, the amount of virus used per well varied from 50$100 \mu \mathrm{l}$. As control, an EGFP-expressing virus was used. This virus showed much higher efficiency and typically $5 \mu \mathrm{l}$ were added per well. Cells were used from $4 \mathrm{~h}$ to $12 \mathrm{~h}$ after infection. Infected cells were identified by EGFP fluorescence and cells exhibiting similar EGFP fluorescence intensities were used.

\subsection{Electrophysiological Recordings and Data Analysis}

Patch-clamping in the whole-cell configuration and $\mathrm{Ca}^{2+}$ microfluorimetry were performed on cultured chromaffin cells according to published protocols (Lindau and Neher, 1988; Nagy et al., 2002; Naraghi et al., 1998; Neher and Marty, 1982). Recordings were obtained at room temperature with Sylgard-coated 4-6M $\Omega$ pipettes at a setup equipped with HEKA EPC-10 amplifier (HEKA Electronik), a Zeiss Axiovert 200 microscope, a Polychrome V monochromator (TILL photonics) and a xenon flash lamp (Rapp OptoElectronics).

The external solution contained $147 \mathrm{mM} \quad \mathrm{NaCl}, 10 \mathrm{mM}$ 2-[4-(2hydroxyethyl)piperazin-1-yl]ethanesulfonic acid (HEPES), $11.1 \mathrm{mM}$ glucose, $2.8 \mathrm{mM} \mathrm{KCl,} 2$ $\mathrm{mM} \mathrm{CaCl}_{2}, 1 \mathrm{mM} \mathrm{MgCl}$ and $3 \mu \mathrm{M}$ tetrodotoxin (TTX) (pH 7.2, osmolarity between 300 and 310 mOsM). In depolarization experiments, TTX was omitted. In flash photolysis experiments, the internal solution contained $109 \mathrm{mM}$ glutamic acid (Sigma, Cat\#49449), 35 mM HEPES (Sigma, Cat\#H3375), $5 \mathrm{mM}$ nitrophenyl-EGTA, $5.65 \mathrm{mM} \mathrm{CaCl} 2,2 \mathrm{mM} \mathrm{Mg-}$ adenosine 5'-triphosphate (ATP), $0.3 \mathrm{mM}$ Na-guanosine 5'-triphosphate (GTP), $0.205 \mathrm{mM}$ fura-4F (Invitrogen), $0.3 \mathrm{mM}$ furaptra (Invitrogen) and $1 \mathrm{mM}$ ascorbic acid (Walter et al., 2010) (pH 7.2 adjusted with $\mathrm{CsOH}$, osmolarity between 290 and $295 \mathrm{mOsM}$ ). The pre-flash $\left[\mathrm{Ca}^{2+}\right]$ is $\approx 300$ to $600 \mathrm{mM}$ (Schonn et al., 2008), and only cells with pre-flash $\left[\mathrm{Ca}^{2+}\right]$ in this range were used for analysis, because the size of the exocytotic burst scales with pre-flash $\left[\mathrm{Ca}^{2+}\right]$ (Voets, 2000). In depolarisation experiments, the internal solution contained $111 \mathrm{mM}$ glutamic acid, $35.5 \mathrm{mM}$ HEPES, $17 \mathrm{mM} \mathrm{NaCl}, 1 \mathrm{mM} \mathrm{MgCl}$, 2 mM Mg-ATP, $0.3 \mathrm{mM} \mathrm{Na-}$ GTP (pH 7.2 adjusted with $\mathrm{CsOH}$, osmolarity $\approx 290-295 \mathrm{mOsM}$ ), and the same concentrations of fura-4F and furaptra as in the flash experiments. The UV-flash stimuli or the depolarisation trains were applied to the cell $\approx 90$ s after the whole cell configuration was 
obtained. The UV-flash was generated by a xenon lamp (Rapp OptoElectronics). Intracellular $\left[\mathrm{Ca}^{2+}\right]$ was monitored both before and after the flash by applying alternating pulses of excitation light at $340 \mathrm{~nm}$ and $380 \mathrm{~nm}$ using a Polychrome V monochromator (TILL photonics) to the cell that had been dialysed with the two ratiometric $\mathrm{Ca}^{2+}$ dyes with different $\mathrm{Ca}^{2+}$ affinities, fura-4F and furaptra (Voets, 2000). Emitted light was recorded and the light intensity was converted to voltage signals by a photodiode. The area of interest was restricted to inside the boundary of the cell. $\left[\mathrm{Ca}^{2+}\right]$ were calculated according to a pre-determined $\mathrm{Ca}^{2+}$ calibration curve generated by measuring cells that has been dialysed with solutions of known $\left[\mathrm{Ca}^{2+}\right]$ (see below). Capacitance recordings and voltage signals for $\left[\mathrm{Ca}^{2+}\right]$ measurements were acquired using a HEKA EPC-10 amplifier and the software Patchmaster (both HEKA Electronik).

For $\mathrm{Ca}^{2+}$ calibration of the system (Voets, 2000; Walter et al., 2010), solutions with different $\left[\mathrm{Ca}^{2+}\right]$ were used as internal solutions. To obtain defined $\left[\mathrm{Ca}^{2+}\right]$, the calibration solutions contained different concentrations of $\mathrm{Ca}^{2+}$-chelating agents. The osmolarity of the solutions was adjusted to $\approx 300 \mathrm{mOsM}$ by slightly altering the concentrations of glutamic acid and HEPES by varying the amount of 2x BP buffer $(250 \mathrm{mM} \mathrm{L-glutamic}$ acid and $80 \mathrm{mM}$ HEPES, $\mathrm{pH}$ adjusted to 7.2 with $\mathrm{CsOH}$ ) added to the solutions. The composition of the solutions and the concentration of free $\mathrm{Ca}^{2+}\left(\left[\mathrm{Ca}^{2+}\right]_{\text {free }}\right)$ of each solution are listed in Table 2.2. The final concentrations of each component of the solutions are listed in Table 2.3. After dialysis (around $90 \mathrm{~s}$ after obtaining whole cell configuration), cells were illuminated with excitation light of alternating wavelengths of $340 \mathrm{~nm}$ and $380 \mathrm{~nm}$. The light emitted by the ratiometric $\mathrm{Ca}^{2+}$ dyes was detected by a photodiode and converted into voltage signals by the amplifier. The ratios of the intensities of the emitted light resulting from $340 \mathrm{~nm}$ and $380 \mathrm{~nm}$ excitation were plotted against the $\left[\mathrm{Ca}^{2+}\right]$ of each solution. $\left[\mathrm{Ca}^{2+}\right]$ of the solutions were calculated using a custom-made macro in the software IgorPro (WaveMetrics). The ratios obtained for each solution were fitted to equation (1)

$$
f(x)=R_{0}+R_{1}\left(\frac{x^{H i l l 1}}{x^{H i l l 1}+K_{D 1}^{H i l l 1}}\right)+R_{2}\left(\frac{x^{H i l l 2}}{x^{H i l l 2}+K_{D 2}^{H i l l 2}}\right)
$$

where $f(x)$ is the ratio of the intensities of emitted light when excited at wavelength $340 \mathrm{~nm}$ to that at $380 \mathrm{~nm}$ at the $\left[\mathrm{Ca}^{2+}\right] x$ of each calibration solution, $K_{D 1}, K_{D 2}$, Hill1 and Hill2 are the microscopic dissociation constants and the Hill coefficients of cooperative binding of $\mathrm{Ca}^{2+}$ for the two $\mathrm{Ca}^{2+}$ dyes, $R_{1}$ and $R_{2}$ are the weightings of the contribution of the two dyes to the 
ratio and $R_{0}$ is the ratio when the $\left[\mathrm{Ca}^{2+}\right]$ is 0 . IgorPro was used to find the relationship between the ratio and $\left[\mathrm{Ca}^{2+}\right]$.

Capacitance data were analysed by fitting a sum of two exponentials to the trace from the time that the flash was given until $0.5 \mathrm{~s}$ after. In cases where no satisfactory fit was obtained, the time point marking the end of the fitting window $\left(t_{\text {fit }}\right)$ was shifted manually to obtain a satisfactory fit. The time constants of two kinetically distinct release components were obtained directly from the fitting. The sizes of the fast burst $\left(\mathrm{B}_{\mathrm{F}}\right)$, the slow burst $\left(\mathrm{B}_{\mathrm{S}}\right)$ and rate of release of the sustained component $r_{\text {sus }}$ (which is assumed to be constant) were calculated according to equations (2)-(4).

$\mathrm{B}_{\mathrm{F}}=\mathrm{A}_{\mathrm{F}} * \mathrm{~A}_{\mathrm{fit}} /\left(\mathrm{A}_{\mathrm{F}}+\mathrm{A}_{\mathrm{S}}\right)$

$\mathrm{B}_{\mathrm{S}}=\mathrm{A}_{\mathrm{S}} * \mathrm{~A}_{\mathrm{fit}} /\left(\mathrm{A}_{\mathrm{F}}+\mathrm{A}_{\mathrm{S}}\right)$

$r_{\text {sus }}=\left(A_{\text {end }}-A_{\text {fit }}\right) /\left(4-t_{\text {fit }}\right)$

where $A_{\text {fit }}$ is the average amplitude of the capacitance trace during $0.05 \mathrm{~s}$ before $t_{\text {fit }}, A_{\text {end }}$ is that from $3.95 \mathrm{~s}$ to $4 \mathrm{~s}$ after the flash, and $\mathrm{A}_{\mathrm{F}}$ and $\mathrm{A}_{\mathrm{S}}$ are the respective amplitudes of the exponential curves given by the fitting. Fitting analysis was performed using the software AxoGraph (AxoGraph Scientific). For rescue of Munc13-1/2 DKO by Baiap3, traces could not be fitted by a sum of exponentials, thus only capacitance increases within $0.5 \mathrm{~s}$ after the flash (the exocytotic burst) and between $0.5 \mathrm{~s}$ to $4 \mathrm{~s}$ (to calculate the rate of sustained release) were analysed. The Student's $t$-test was used for statistical analyses in all experiments. 


\begin{tabular}{|c|c|c|c|c|c|c|c|c|}
\hline \multirow[t]{2}{*}{ Components } & \multicolumn{8}{|c|}{ Volumes of component in each solution $(\mu \mathrm{l})$} \\
\hline & Solution 1 & Solution 2 & Solution 3 & Solution 4 & Solution 5 & Solution 6 & Solution 7 & Solution 8 \\
\hline Nucleotides (20 Mg-ATP, 3 Na-GTP) & 8 & 8 & 8 & 8 & 8 & 8 & 8 & 8 \\
\hline BAPTA $(100)$ & 16 & 6.4 & 3.6 & - & - & - & - & - \\
\hline Ca-BAPTA (100) & - & 9.6 & 12.4 & - & - & - & - & - \\
\hline DPTA (100) & - & - & - & 30.4 & 27.6 & 24.4 & 16.4 & - \\
\hline Vitamin C (40) & 2 & 2 & 2 & 2 & 2 & 2 & 2 & 2 \\
\hline $\mathrm{H}_{2} \mathrm{O}$ & 16.8 & 21.4 & 22.9 & 10 & 11.1 & 12.2 & 16.8 & 17.2 \\
\hline Final volume $(\mu \mathrm{l})$ & 80 & 80 & 80 & 80 & 80 & 80 & 80 & 80 \\
\hline Calculated $\left[\mathrm{Ca}^{2+}\right]_{\text {free }}$ & $0 \mathrm{nM}$ & $334.4 \mathrm{nM}$ & $757.3 \mathrm{nM}$ & $3.906 \mu \mathrm{M}$ & $12.39 \mu \mathrm{M}$ & $24.49 \mu \mathrm{M}$ & $74.63 \mu \mathrm{M}$ & $10.35 \mathrm{mM}$ \\
\hline
\end{tabular}

Table 2.2 Composition of solutions used for calcium calibration. The leftmost column lists the stock solutions used as components of solution 1-8 for calcium calibration. Concentrations of stock solutions were indicated in brackets in unit mM. 2x BP solution is $250 \mathrm{mM}$ L-glutamic acid, $80 \mathrm{mM}$ HEPES (pH 7.2 with CsOH). ATP = adenosine triphosphate; GTP = guanosine triphosphate; BAPTA = 1,2-bis(o-aminophenoxy)ethane$\mathrm{N}, \mathrm{N}, \mathrm{N}$ ',N'-tetraacetic acid; DPTA = diethylenetriaminepentaacetic acid. 


\begin{tabular}{|c|c|c|c|c|c|c|c|c|}
\hline Species & Solution 1 & Solution 2 & Solution 3 & Solution 4 & Solution 5 & Solution 6 & Solution 7 & Solution 8 \\
\hline $\mathrm{Mg}^{2+}$ & 2 & 2 & 2 & 2 & 2 & 2 & 2 & 2 \\
\hline ATP & 2 & 2 & 2 & 2 & 2 & 2 & 2 & 2 \\
\hline Fura-4F & 0.205 & 0.205 & 0.205 & 0.205 & 0.205 & 0.205 & 0.205 & 0.205 \\
\hline Furaptra & 0.3 & 0.3 & 0.3 & 0.3 & 0.3 & 0.3 & 0.3 & 0.3 \\
\hline BAPTA & 20 & 20 & 20 & 0 & 0 & 0 & 0 & 0 \\
\hline DPTA & 0 & 0 & 0 & 40 & 40 & 40 & 40 & 0 \\
\hline $\mathrm{Ca}^{2+}$ & 0 & 12 & 15.5 & 2 & 5.5 & 9.5 & 19.5 & 12.5 \\
\hline
\end{tabular}

Table 2.3 Concentrations of magnesium ions, calcium ions, ATP, fura-4F, furaptra, BAPTA and DPTA in each solution for calcium calibration. 


\section{Results}

\subsection{Expression of Munc13 Isoforms in the Adrenal Gland}

Expression levels of each Munc13 isoform in adrenal glands from WT mice and KO littermates as control were examined using Western blotting with isoform-specific antibodies (Cooper et al., 2012). Munc13-1 KO P0 pups are weak and die within a few hours after birth (Augustin et al., 1999b), and mice deficient for both Munc13-1 and Munc13-2 are often stillborn (Varoqueaux et al. 2002). Thus, perinatal animals (E18 or P0) were used throughout this study, except where indicated otherwise. VCP was used as the loading control for brain and adrenal homogenates, because its expression levels are similar in these two organs (Nagy et al., 2008; Schonn et al., 2008). GAPDH was used as a loading control for blotting against Munc13-4, because the apparent molecular weights of Munc13-4 and VCP are too similar (both $\approx 100 \mathrm{kDa}$ ).

Immunoblotting showed that Munc13-1 was strongly expressed in brains from E18 WT animals, while in the adrenal gland expression is very weak (Fig. 3.1A). Expression in both the brain and adrenal gland were abolished in tissues from littermate Munc13-1 KO mice. Truncated protein products of Munc13-1 were detected at $\approx 120 \mathrm{kDa}$ in brains from Munc13-1 KO animals (Fig. 3.1A). These were also expressed in adrenal glands from Munc13-1 KO mice, but at much lower levels. ubMunc13-2 expression in brain from P0 WT animals is strong, and its expression in the adrenal gland is weaker than that in brain but readily detectable (Fig. 3.1B). The expression of ubMunc13-2 was abolished in Munc13-2 KO mice. Truncated products of $\approx 70 \mathrm{kDa}$ could be observed in Munc13-2 KO brains (data not shown). They were also expressed in adrenal glands of these mice with weaker expression levels. Attempts by my colleagues to characterise these fragments could not clearly define their identities, however the N-terminal ubMunc13-2 fragment is thought to be non-functional (Cooper et al., 2012). bMunc13-2 expression was observed in P0 WT brain, but not detected in P0 WT adrenal glands (Fig. 3.1B).

Munc13-3 expression was detected in P0 brain from WT animals but not in adrenal glands (Fig. 3.1C). Baiap3 expression was detected in WT P0 brain, was weaker in adrenal glands than in brain (Fig. 3.1D), and was abrogated in Baiap3 KO mice. A truncated product with a slightly smaller size was observed in P0 Baiap3 KO brain, its expression was not easily detectable in adrenal gland but cannot be ruled out, as overnight exposure revealed bands of similar sizes as the truncated product detected in brain (Supplementary Fig. 1, 
overnight exposure). In brain, the expression of this truncated product peaked at P7 and decreased until it disappeared in adult mice (Wojcik et al., 2013). Munc13-4 expression could be detected in adult WT lung tissues and was abrogated in the Munc13-4 KO mice, while it could not be detected in WT adult adrenal glands (Fig. 3.1E).

In E18 Munc13-1/2 DKO mice, expression of Munc13-1 and ubMunc13-2 was abrogated, while in littermate WT animals, expression of these two isoforms was intact (Fig. 3.1F). The truncated Munc13-1 and Munc13-2 fragments that were detected in the respective single KO lines were also observed in the Munc13-1/2 DKO mice (Fig. 3.1F for Munc13-1 and data not shown for Munc13-2). 


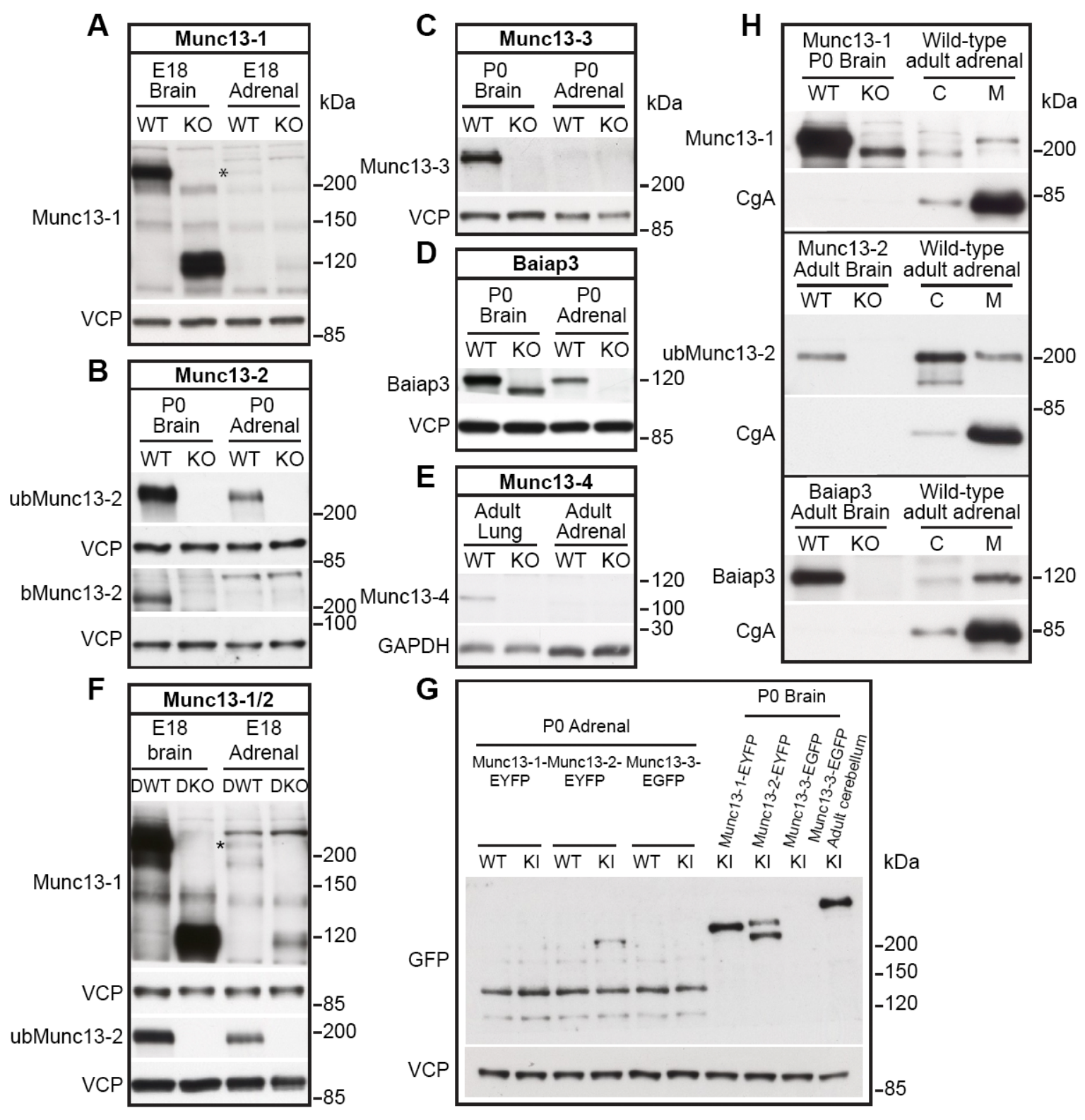

Fig. 3.1 Expression of Munc13 isoforms by Western blotting. Antibodies used are indicated on the left. (A) Expression of Munc13-1 in brain and adrenal glands of E18 Munc13-1 WT and KO mice. Asterisk indicates the signal for Munc13-1 protein which is absent in homogenates from Munc13-1 KO mice. (B) Expression of ubMunc13-2 and bMunc13-2 in brain and adrenal glands of P0 Munc13-2 WT and KO mice. (C) Expression of Munc13-3 in P0 Munc13-3 WT and KO mice. (D) Expression of Baiap3 in P0 Baiap3 WT and KO mice. (E) Expression of Munc13-4 in lung and adrenal gland in adult Munc13-4 WT and KO mice. (F) Expression of Munc13-1 and ubMunc13-2 in brain and adrenal glands of Munc13-1/2 double WT (DWT) and DKO mice. Asterisk indicates the signal for Munc13-1 protein that is absent from Munc13-1/2 DKO mice. (G) Expression of Munc13-1, ubMunc13-2, bMunc13-2 and Munc13-3 in adrenal glands from P0 WT and littermate KI Munc13-1-EYFP, Munc13-2EYFP and Munc13-3-EGFP mice detected by anti-GFP antibody. (H) Expression of Munc13-1, ubMunc13-2 and Baiap3 in the adrenal cortex and medulla from adult WT mice. 
In the expression analysis described above, different antibodies were used to probe the expression levels of the Munc13 isoforms. In order to circumvent differences in antibody affinity and directly compare the expression levels of the Munc13-1, Munc13-2 and Munc133 isoforms, Western blotting was performed on adrenal homogenates prepared from P0 Munc13-1-EYFP, Munc13-2-EYFP, Munc13-3-EGFP KI mice along with their respective WT littermates as control using an anti-EGFP antibody (Fig. 3.1G). Expression of the tagged Munc13 isoforms was easily detectable in brain homogenates from the respective KI mice. In adrenal gland homogenate, only Munc13-2-EYFP was detectable using an anti-EGFP antibody, whereas no signal was observed for Munc13-1-EYFP and Munc13-3-EGFP. These data confirm that in mouse perinatal adrenal gland ubMunc13-2 is the isoform with the highest expression level.

Chromaffin cells reside in the adrenal medulla. To test if Munc13-1, ubMunc13-2 and Baiap3 are present in the adrenal medulla and/or the adrenal cortex, adrenal cortical and medullary homogenates were prepared from adult WT animals. The separation of adrenal cortex and medulla by dissection was verified by blotting for chromogranin $\mathrm{A}(\mathrm{CgA})$, which expresses exclusively in the adrenal medulla but not in the cortex (Hawkins et al., 1989). The medullary homogenate was highly enriched in $\mathrm{CgA}$, while there were only low levels of $\mathrm{CgA}$ in the cortical homogenate, indicating a relatively clean separation of the two types of tissue (Fig. 3.1H). Munc13-1 was clearly detected in the medulla, whereas cortical Munc13-1 levels appear to be very low. Baiap3 was also mostly present in the medulla, with much lower levels detected in the cortical sample. In contrast to this, ubMunc13-2 levels were higher in the cortex than in the medulla. Although this experiment used adult adrenal glands, which may show a pattern of expression that differs from that of perinatal glands, this suggests that Munc13-1 and Baiap3 are predominantly expressed in chromaffin cells, whereas a significant fraction of the ubMunc13-2 detected in whole adrenal gland is due to innervation of the adrenal cortex by Munc13-2 positive synapses.

To check for possible compensatory expression of other Munc13 isoforms as a consequence of deletion of a particular isoform, Western blotting for Munc13-1, ubMunc132, bMunc13-2, Munc13-3 and Baiap3 was performed in brain and adrenal gland homogenates of Munc13-1, Munc13-2, Munc13-3 and Baiap3 single KO animals (Supplementary Fig. 2). No compensatory changes were observed. 
In summary, Munc13-1, ubMunc13-2 and Baiap3 are expressed in perinatal adrenal glands, and expression is abolished in the respective KO animals. ubMunc13-2 and Baiap3 expression is high, while Munc13-1 expression is low. The presence of Munc13-1 and Baiap3 in the adrenal gland is contributed mainly by expression in the medulla, whereas ubMunc13-2 levels are higher in cortex than in medulla. The expression of bMunc13-2, Munc13-3 and Munc13-4 was not detected.

\subsection{LDCV Exocytosis is Intact in Munc13-1 KO Chromaffin Cells}

Next, to examine the role of individual Munc13 isoforms in LDCV exocytosis, $\mathrm{Ca}^{2+}$ triggered fusion of LDCVs with the plasma membrane was monitored using capacitance recordings in chromaffin cells after flash photolysis of caged $\mathrm{Ca}^{2+}$. Capacitance traces obtained from littermate E18 Munc13-1 ${ }^{+/+}(n=41)$ and Munc13-1/-- $(n=45)$ chromaffin cells are shown in Fig. 3.2A, B. Averaged traces from the two groups were overlaid for comparison in Fig. 3.2C. Intracellular $\left[\mathrm{Ca}^{2+}\right]$ before and after the flash did not differ significantly between genotypes. The shapes of the averaged traces were similar, except for a seemingly larger release from Munc13-1/- cells. Individual traces normalised at $1 \mathrm{~s}$ after the flash for the two groups superposed each other, showing that the kinetics of release did not differ between the two groups (Fig. 3.2D). Upon fitting each trace within $500 \mathrm{~ms}$ after the flash with a sum of two exponentials the sizes and time constants of individual release components were obtained (Fig. 3.2E). The fast burst $\left(112.81 \pm 12.22 \mathrm{fF}\right.$ for Munc13-1 ${ }^{+/+}$; $119.41 \pm 10.12 \mathrm{fF}$ for Munc13-1 $\left.{ }^{-/-}\right)$, the rate of sustained release $(53.38 \pm 4.01 \mathrm{fF} / \mathrm{s}$ for Munc13-1 $1^{+/+} ; 60.07 \pm 3.79 \mathrm{fF} / \mathrm{s}$ for Munc13-1/- $)$ and the time constants of the fast and slow burst components (fast time constants: $19.00 \pm 1.41 \mathrm{~ms}$ for Munc13-1 ${ }^{+/+}, 19.06 \pm 1.26 \mathrm{~ms}$ for Munc13-1 ${ }^{-/}$; slow time constants: $488.84 \pm 49.46 \mathrm{~ms}$ for Munc13-1 ${ }^{+/+}, 499.86 \pm 54.09 \mathrm{~ms}$ for Munc13-1 $1^{-/}$) were not significantly different from each other. However the slow burst component of Munc13-1 $1^{-/-}$cells was significantly increased by $\approx 24 \%(124.99 \pm 7.8713 \mathrm{fF}$ for Munc13-1 $1^{+/+} ; 155.42 \pm 10.74 \mathrm{fF}$ for Munc13-1 $1^{-/}$; Student's $t$-test, $p<0.05$ ). 


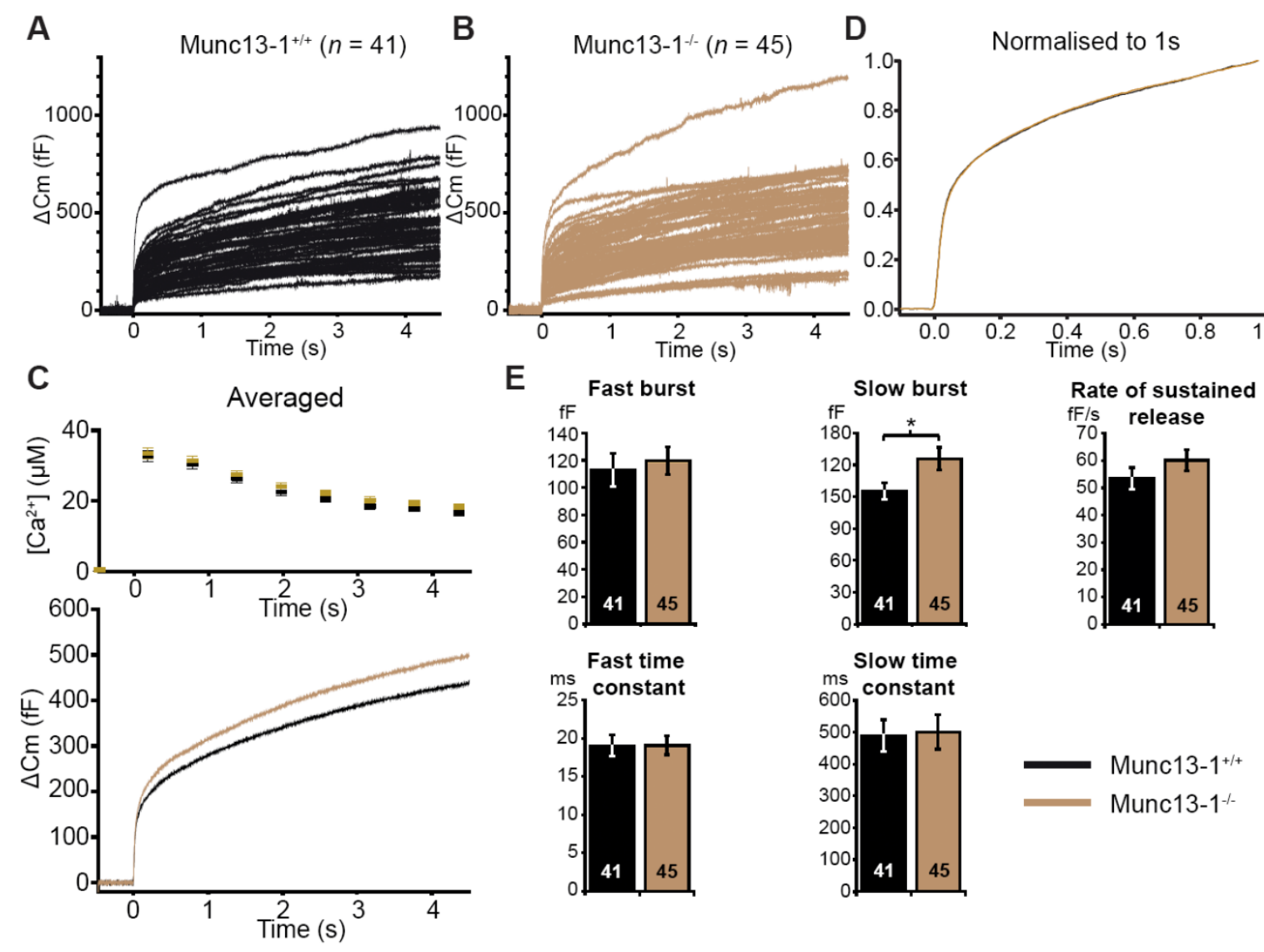

Fig. 3.2 LDCV exocytosis measured by capacitance changes during flash photolysis of caged $\mathrm{Ca}^{2+}$ in E18 Munc13-1 $1^{+/+}(n=41)$ and Munc13-1 $1^{-/-}(n=45)$ chromaffin cells. The flash occurred at $t=0 \mathrm{~s}$. Traces from (A) Munc13-1 ${ }^{+/+}$cells, (B) littermate Munc13-1 $1^{-/}$cells. (C) Averaged $\left[\mathrm{Ca}^{2+}\right]$ and capacitance traces for both groups before and after the flash. (D) Normalised traces within the first second after the flash. (E) Sizes and time constants of the two components of exocytotic burst and rate of sustained release $(*, p<0.05)$.

\subsection{LDCV Exocytosis is Intact in Baiap3 KO Chromaffin Cells}

Baiap3 is expressed in the medulla of adrenal glands (Fig. 1D, G). To investigate a possible role for Baiap3 in priming of releasable LDCVs, exocytosis from P0 Baiap3 ${ }^{-/-}$ chromaffin cells was examined using littermate Baiap3 ${ }^{+/+}$cells as control (Fig. 3.3). The shapes of capacitance traces from Baiap $3^{+/+}(n=56)$ and Baiap3 $3^{-/-}(n=59)$ cells were similar, and intracellular $\left[\mathrm{Ca}^{2+}\right]$ before and after the flash did not differ between experimental groups (Fig. 3.3A-C). Traces from the two groups normalised at $1 \mathrm{~s}$ superposed each other, indicating that their release kinetics were not different (Fig. 3.3D). Upon fitting by a sum of two exponentials, all pool sizes (fast burst: $88.76 \pm 6.14 \mathrm{fF}$ for Baiap3 ${ }^{+/+}, 105.74 \pm 8.20 \mathrm{fF}$ for Baiap3 $^{-/-}$; slow burst: $134.24 \pm 7.29 \mathrm{fF}$ for Baiap3 $3^{+/+}, 142.25 \pm 8.16 \mathrm{fF}$ for Baiap3 ${ }^{-/}$), rate of sustained release $\left(53.13 \pm 3.44 \mathrm{fF} / \mathrm{s}\right.$ for Baiap $3^{+/+} ; 56.69 \pm 3.59 \mathrm{fF} / \mathrm{s}$ for Baiap3 $\left.{ }^{-/}\right)$and 
kinetics of release components (fast time constant: $18.06 \pm 0.85 \mathrm{~ms}$ for Baiap $3^{+/+}, 18.33 \pm$ $0.94 \mathrm{~ms}$ for Baiap3 $3^{--}$; slow time constant: $390.11 \pm 22.97 \mathrm{~ms}$ for Baiap3 ${ }^{+/+}, 464.58 \pm 40.69$ ms for Baiap3 $3^{-/}$) were not observed to be significantly different. Thus, Baiap3 is dispensable for LDCV release in chromaffin cells triggered by a step increase in $\left[\mathrm{Ca}^{2+}\right]$.
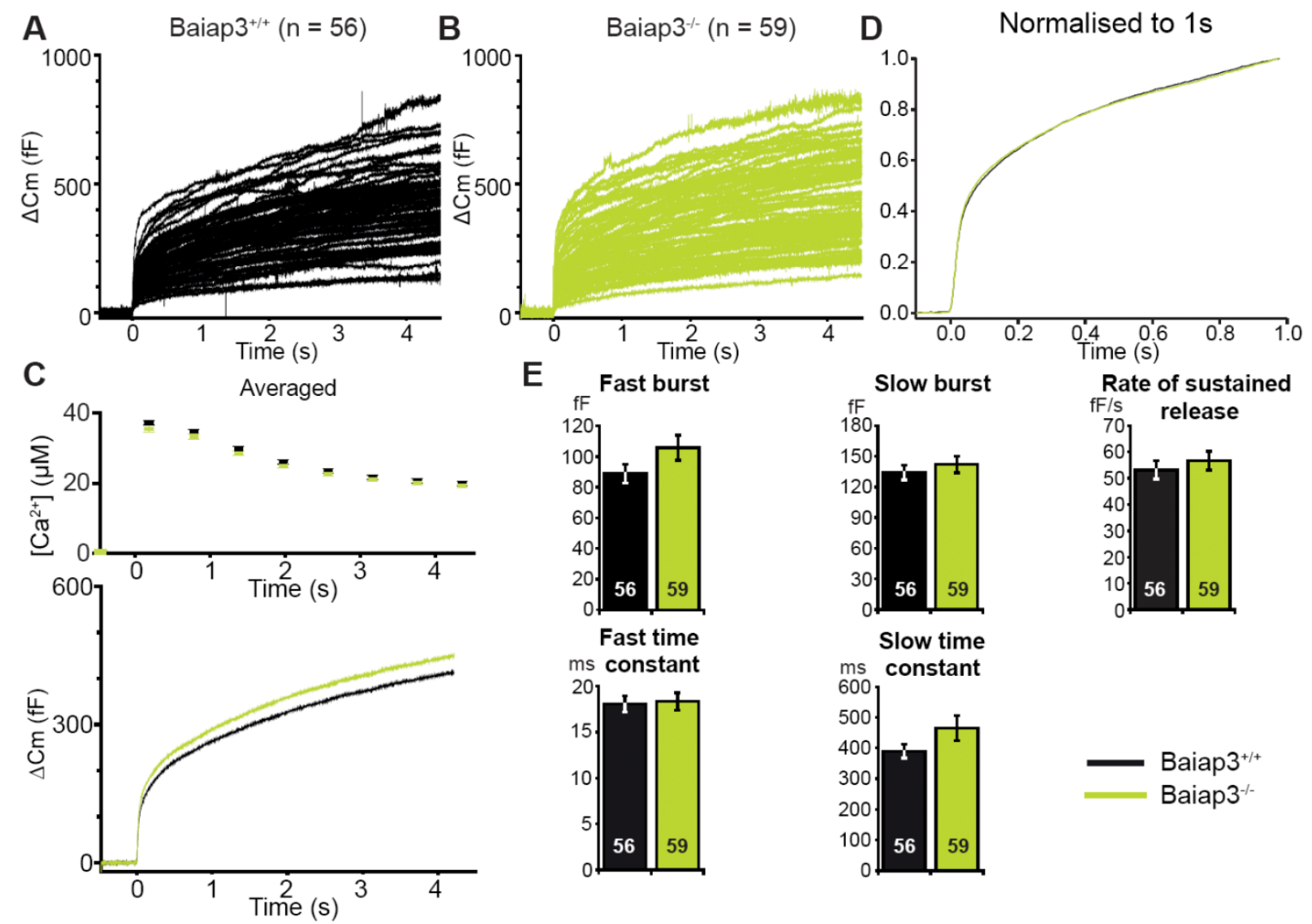

Rate of sustained

$\mathrm{fF} / \mathrm{s}$ release

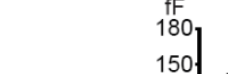

70
60
50

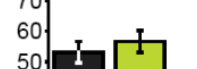

40

40
30
20.

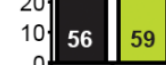

\section{.}


Munc13-3 KO chromaffin cells (Fig. 3.4A-C). No differences were observed in pool sizes (Fig. 3.4E, fast burst: $83.82 \pm 7.12 \mathrm{fF}$ for Munc13-3 $3^{+/+}, 84.35 \pm 7.20 \mathrm{fF}$ for Munc13-3 ${ }^{-/-}$; slow

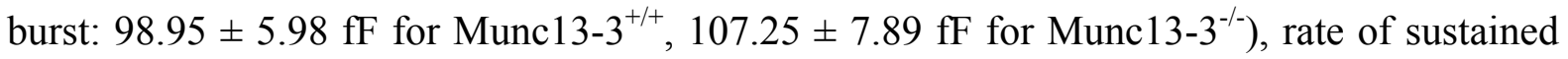
release $\left(43.22 \pm 3.24 \mathrm{fF} / \mathrm{s}\right.$ for Munc13-3 $3^{+/+} ; 46.42 \pm 2.83 \mathrm{fF} / \mathrm{s}$ for Munc13-3 $\left.3^{-/-}\right)$and kinetics of release (Fig. 3.4D, E, fast time constant: $18.85 \pm 0.92 \mathrm{~ms}$ for Munc13-3 ${ }^{+/+}, 20.35 \pm 1.21 \mathrm{~ms}$ for Munc13-3 ${ }^{-/}$; slow time constant: $413.21 \pm 27.24 \mathrm{~ms}$ for Munc13-3 $3^{+/+}, 405.96 \pm 29.31 \mathrm{~ms}$ for Munc13-3 ${ }^{-/-}$) between Munc13-3 $3^{+/+}$and Munc13-3 $3^{-/-}$cells. Thus, Munc13-3 is not required in LDCV release triggered by a step increase of $\left[\mathrm{Ca}^{2+}\right]$.

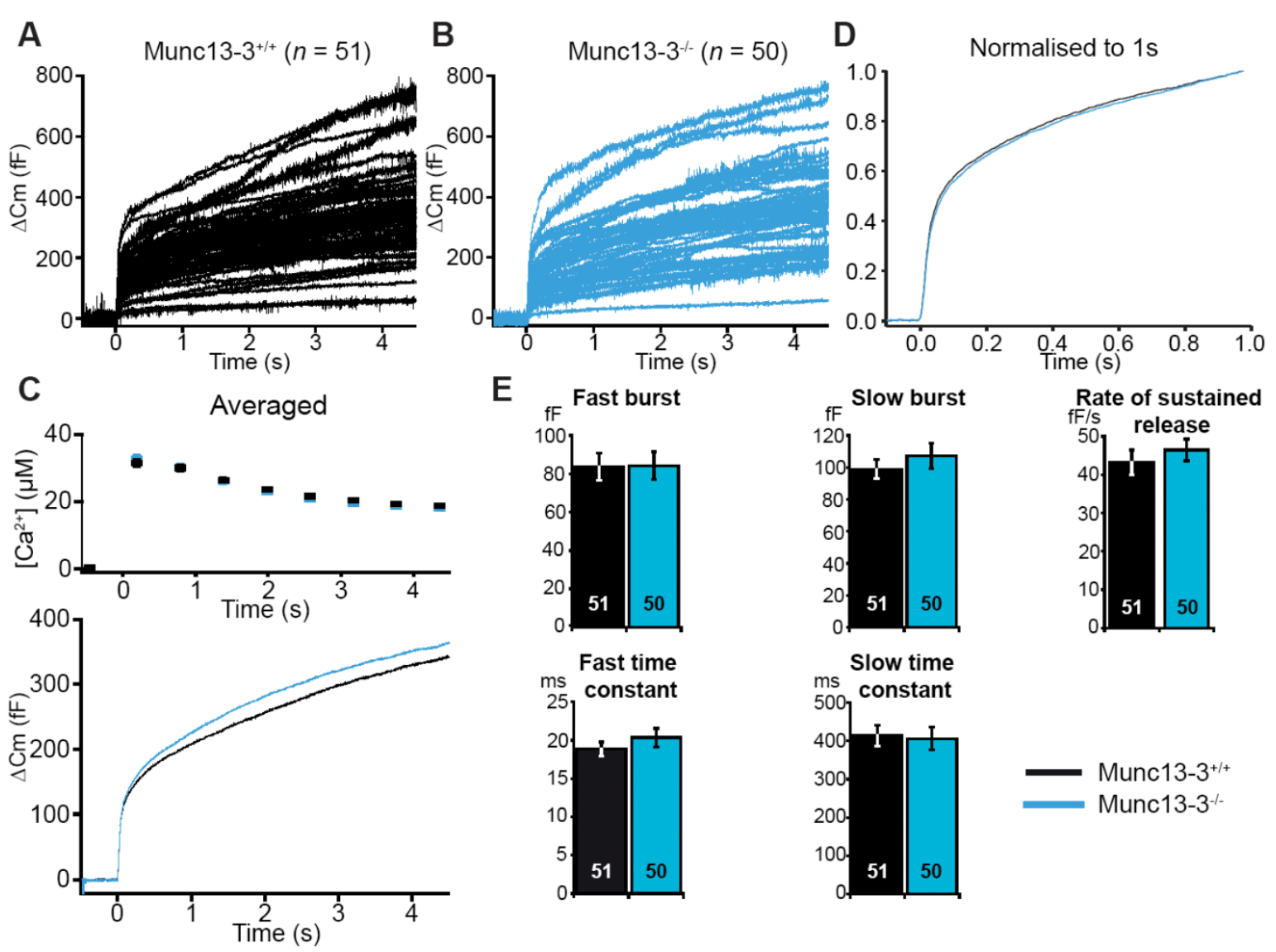

Fig. 3.4 LDCV exocytosis measured by capacitance changes during flash photolysis of caged $\mathrm{Ca}^{2+}$ in P0 Munc13-3 $3^{+/+}(n=51)$ and Munc13-3 $3^{-/-}(n=50)$ chromaffin cells. Traces from (A) Munc13-3 $3^{+/+}$cells, (B) littermate Munc13-3 $3^{-/-}$cells. (C) Averaged $\left[\mathrm{Ca}^{2+}\right]$ and capacitance traces for both groups before and after the flash. (D) Normalised traces within the first second after the flash. (E) Sizes and time constants of the two components of exocytotic burst and rate of sustained release. 


\section{5 ubMunc13-2 is Required for LDCV Exocytosis in Chromaffin Cells and a Role for Munc13-1 Becomes Apparent in the Absence of Munc13-2}

Aside from Baiap3, ubMunc13-2 is the Munc13 isoform with the highest expression in perinatal adrenal gland (Fig. 3.1B, F, G). Since deletion of Baiap3 did not have any effect on exocytosis in flash photolysis experiments (Fig. 3.3), we focused on analysing the function of ubMunc13-2 and Munc13-1 in LDCV exocytosis in chromaffin cells. Capacitance measurement during flash photolysis of caged $\mathrm{Ca}^{2+}$ was performed in chromaffin cells from E18 littermates that were deficient for either Munc13-1, or for Munc13-2 or for both isoforms (Munc13-1/2 DKO) (Fig. 3.5A-E). In this experiment, Munc13-1 ${ }^{+/+}$Munc13$2^{+/-}$and Munc13-1 $1^{+/-}$Munc13-2 ${ }^{+/-}$cells were used as control $(n=106)$. Data were collected from genotype groups available for a given litter and were pooled for analysis. A comparison of both pre-flash and post-flash $\left[\mathrm{Ca}^{2+}\right]$ measured from this experiment is shown in Supplementary Table 1. Pre-flash $\left[\mathrm{Ca}^{2+}\right]$ of all experimental groups were not significantly different from each other. Post-flash $\left[\mathrm{Ca}^{2+}\right]$ of the five groups were similar, but some exceptions exist. The post-flash $\left[\mathrm{Ca}^{2+}\right]$ of Munc13-1/2 DKO cells was higher than in control cells in later time points in the recordings, but since we could observe a large reduction of the rate of sustained release in DKO cells compared to control (see below), the higher post-flash $\left[\mathrm{Ca}^{2+}\right]$ is not expected to affect sustained release significantly compared to the effect of gene deletion. The same applies to the comparison between Munc13-1 $1^{-/-}$Munc13-2 ${ }^{+/-}$cells and Munc13-1 ${ }^{+/+}$Munc13-2 $2^{-/-}$cells (see Supplementary Table 1). The post-flash $\left[\mathrm{Ca}^{2+}\right]$ of Munc13-1 ${ }^{+/-}$Munc13-2 ${ }^{-/-}$cells (first post-flash $\left[\mathrm{Ca}^{2+}\right]$ measurement $=29.98 \pm 0.633 \mu \mathrm{M}$ ) were significantly lower than control cells (first post-flash $\left[\mathrm{Ca}^{2+}\right]$ measurement $=34.34 \pm 0.73$ $\mu \mathrm{M})$. The post-flash $\left[\mathrm{Ca}^{2+}\right]$ differences were within $4.5 \mu \mathrm{M}$. Since both Munc13- $1^{+/+}$Munc13$2^{-/-}$and Munc13-1/2 DKO cells showed large reductions of the rate of sustained release compared to controls, and the effect on Munc13-1 $1^{+/-}$Munc13-2 $2^{-/-}$cells was intermediate between them (see below), it appears that genotype differences exerted a larger effect than the lower post-flash $\left[\mathrm{Ca}^{2+}\right]$ in these cells on sustained release. Considering the exocytotic burst, since post-flash $\left[\mathrm{Ca}^{2+}\right]$ of between $10 \mu \mathrm{M}$ and $60 \mu \mathrm{M}$ is not expected to affect the size of the exocytotic burst and the relative sizes of RRP and SRP (Voets, 2000), the difference in post-flash $\left[\mathrm{Ca}^{2+}\right]$ observed here is not expected to affect the analysis of the exocytotic burst. However, the lower post-flash $\left[\mathrm{Ca}^{2+}\right]$ in Munc13-1 $1^{+/-}$Munc13-2 ${ }^{-/-}$cells probably contributes to the slightly longer but not significantly different fast and slow time constants of this group compared to other experimental groups (Fig. 3.5G) (Voets, 2000). 
In Munc13-2 KO cells (Munc13-1 ${ }^{+/+} \operatorname{Munc13-2}^{-/-}, n=46$; Munc13-1 ${ }^{+/-}$Munc13-2-/-,$n$ = 36; Munc13-1 $1^{-/-}$Munc13-2/-,$n=66$ ), irrespective of Munc13-1 genotype, a drastic reduction in release is observed (Fig. 3.5 C-E) as compared to cells from control animals ( $n=$ 106, Fig. 3.5A) and from Munc13-1/-Munc13-2 ${ }^{+/-}$animals $(n=42$, Fig. 3.5B). Examination of overlaid averaged traces from the 5 groups of different genotypes showed that LDCV release from the control group and Munc13-1/- Munc13-2 $2^{+/-}$cells were almost identical as shown by their near superposition, whereas release from Munc13-2 KO cells was diminished. It has been shown by Western blotting that in the Munc13-1/2 DKO mouse line used in this experiment, the amount of Munc13-1 expression is reduced in Munc13-1 heterozygous brain by $\approx 30-50 \%$ as compared to Munc13-1 WT brain (Augustin et al., 1999b; Varoqueaux et al., 2002) and Munc13-2 expressed in brain heterozygous for Munc13-2 is reduced by $\approx 50 \%$ as compared to Munc13-2 WT (Varoqueaux et al., 2002). In the absence of Munc13-2, cells with different Munc13-1 genotypes showed heterogeneous release properties, with slight reductions in release that were dependent on the amount of Munc13-1 protein present.

Upon fitting a sum of two exponentials to individual traces, it was observed that deletion of Munc13-2 caused highly significant reductions in the sizes of the fast burst, slow burst and sustained release component (Fig. 3.5G, Table 3.1) as compared to the control group. The fast bursts of Munc13- $1^{+/+}$Munc13-2 ${ }^{-/-}$, Munc13- $1^{+/-}$Munc13-2/- and Munc13-1/2 DKO cells were only 40\%,31\% and 29\% of control cell levels, respectively (all $p<0.001$ ). The slow bursts of the above three groups were only $48 \%, 44 \%$ and $34 \%$ of control cell levels, respectively (all $p<0.001$ ). The rates of sustained release of the three groups were reduced even more drastically, and were only $28 \%, 23 \%$ and $13 \%$ of control cell levels, respectively (all $p<0.001$ ). The kinetics of release from Munc13-2 KO cells was not significantly different from control cells (Fig. 3.5G, H, Table 3.1). Since ubMunc13-2, but not bMunc13-2, was detected in perinatal adrenal glands, ubMunc13-2 is essential in priming LDCVs for release in chromaffin cells. 


\begin{tabular}{|c|c|c|c|c|c|}
\hline Genotype & Fast burst (fF) & $\begin{array}{l}\text { Slow burst } \\
\text { (fF) }\end{array}$ & $\begin{array}{l}\text { Fast time } \\
\text { constant (ms) }\end{array}$ & $\begin{array}{l}\text { Slow time } \\
\text { constant (ms) }\end{array}$ & $\begin{array}{l}\text { Rate of } \\
\text { sustained } \\
\text { release }(\mathrm{fF} / \mathrm{s})\end{array}$ \\
\hline $\begin{array}{l}\text { Munc } 13-1^{+/+} \text {or }^{+-} \\
\text {Munc13-2 }\end{array}$ & $72.02 \pm 3.75$ & $89.54 \pm 3.63$ & $18.92 \pm 0.78$ & $454.69 \pm 19.64$ & $30.09 \pm 1.53$ \\
\hline Munc13-1-1-Munc13-2 & $74.14 \pm 6.31$ & $96.12 \pm 5.62$ & $15.81 \pm 0.87$ & $445.54 \pm 33.37$ & $31.33 \pm 1.98$ \\
\hline Munc13-1 ${ }^{+/+}$Munc $13-2^{-1-}$ & $28.35 \pm 2.97$ & $42.77 \pm 3.49$ & $16.53 \pm 1.14$ & $398.44 \pm 30.63$ & $8.55 \pm 0.97$ \\
\hline Munc13-1+-Munc13-2-- & $22.53 \pm 2.21$ & $39.31 \pm 3.50$ & $18.55 \pm 1.68$ & $480.54 \pm 45.13$ & $7.00 \pm 0.90$ \\
\hline Munc13-1-1-Munc $13-2^{-1-}$ & $21.19 \pm 3.63$ & $30.75 \pm 2.97$ & $17.67 \pm 1.50$ & $434.86 \pm 32.43$ & $3.88 \pm 0.42$ \\
\hline
\end{tabular}

Table 3.1 Sizes and time constants of the fast burst, slow burst and the rate of sustained release for chromaffin cells from mice with different Munc13-1 and Munc13-2 genotypes.
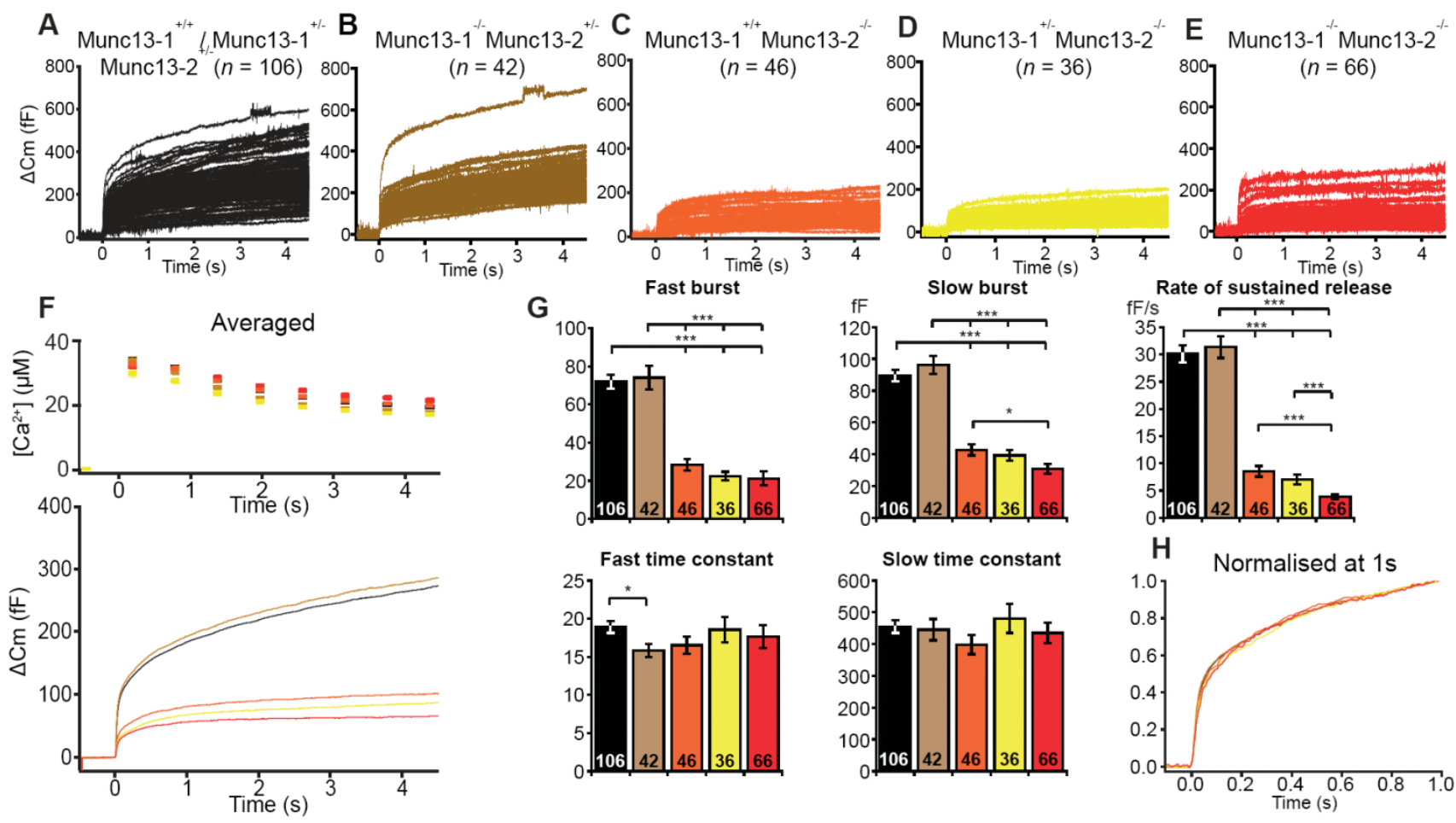

Fig. 3.5 LDCV exocytosis as measured by capacitance changes during flash photolysis of caged $\mathrm{Ca}^{2+}$ in chromaffin cells from E18 Munc13-1/2 DKO, Munc13-1 KO and Munc13-2 KO littermates. Traces from (A) Munc13-1 ${ }^{+/+}$Munc13-2 $2^{+/-}$and Munc13-1 $1^{+/}$Munc13-2 ${ }^{+/-}$cells $(n=106)$, (B) Munc13-1/- Munc13-2 $2^{+/-}$cells $(n=42)$, (C) Munc13-1 ${ }^{+/+}$Munc13-2 ${ }^{-/-}$cells $(n=$ 46), (D) Munc13-1 $1^{+/-}$Munc13-2 ${ }^{-/}$cells $(n=36)$, and (E) Munc13-1/2 DKO cells $(n=66)$. (F) Averaged $\left[\mathrm{Ca}^{2+}\right]$ and capacitance traces for all five groups before and after the flash. $(\mathrm{G})$ Sizes and time constants of the two components of exocytotic burst and rate of sustained release for all five genotype groups $(* * *, p<0.001$; $*, p<0.05)$. (H) Normalised traces within the first second after the flash.

In the single Munc13-1 KO line exocytosis of the SRP or of the sustained component was not reduced (Fig. 3.2E). However, in the absence of ubMunc13-2, the deletion of 
Munc13-1 did result in a reduction of LDCV release (Fig. 3.5G, Table 3.1). The fast bursts of Munc13-1 ${ }^{+/+}$Munc13-2 ${ }^{-/-}$cells and that of Munc13-1/2 DKO cells were not significantly different, but the slow burst of DKO cells was significantly smaller than in Munc13$1^{+/+}$Munc13-2 ${ }^{-/-}$cells (72\% of control levels, $p<0.05$, Fig. 3.5G, Table 3.2). The effect was stronger for the sustained component, with DKO cells releasing at only $45 \%$ of Munc13$1^{+/+}$Munc13-2 ${ }^{-/}$cells $(p<0.001$, Fig. 3.5G, Table 3.2). An intermediate reduction was observed for Munc13-1//-Munc13-2/- cells compared to the DKO, with DKO cells releasing at $55 \%$ of Munc13-1 $1^{+/-}$Munc13-2 ${ }^{-/-}$cells $(p<0.001$, Fig. 3.5G). Table 3.2 illustrates the contribution of Munc13-1 to release in the absence of Munc13-2. There were no changes to the kinetics of release upon Munc13-1 deletion in the Munc13-2 KO background (Fig. 3.5G, $\mathrm{H})$. These data demonstrate that although ubMunc13-2 provides the largest contribution to regulating LDCV exocytosis in chromaffin cells, in its absence, Munc13-1 participates in priming LDCVs for release, affecting the slow burst and the release of vesicles during ongoing stimulation at high intracellular $\left[\mathrm{Ca}^{2+}\right]$.

\begin{tabular}{cccc}
\hline $\begin{array}{c}\text { In Munc13-2 } \\
\text { background }\end{array}$ & Fast burst & Slow burst & $\begin{array}{c}\text { Rate of sustained } \\
\text { release }\end{array}$ \\
\hline Munc13-1 $^{+/+}$ & $100 \%$ & $100 \%$ & $100 \%$ \\
Munc13-1 $^{+/-}$ & $\approx 79 \%$ & $\approx 92 \%$ & $\approx 82 \%$ \\
Munc13-1 $^{-/-}$ & $\approx 75 \%$ & $\approx 72 \%$ & $\approx 45 \%$ \\
\hline
\end{tabular}

Table 3.2 Relative sizes of the Munc13-1 dependent fast burst, slow burst and rate of sustained release in the absence of Munc13-2. Release in Munc13-1 ${ }^{+/+}$Munc13-2 $2^{-/-}$ chromaffin cells was set to $100 \%$.

Pool sizes of Munc13-1/- Munc13-2 $2^{+/-}$cells and the rate of sustained release were not different from control cells (Fig. 3.5G, Table 3.1). Munc13-1 $1^{-/}$Munc13-2 $2^{+/-}$cells showed a slightly shorter fast time constant as compared to the control groups (Fig. 3.5G, $p<0.05$ ), but a corresponding shift in the normalised trace was not observed (Fig. 3.5H). Due to the comparison of multiple genotypes, the data shown in Fig. 3.5 include recordings obtained from non-littermates. When recordings of Munc13-1/-Munc13-2 ${ }^{+/-}(n=33)$ cells and control cells $(n=30)$ that were obtained from littermate animals on the same day were analysed separately, their averaged fast time constants $\left(16.02 \pm 1.02 \mathrm{~ms}\right.$ for Munc13-1 $1^{-/-}$Munc13-2 ${ }^{+/}$; $18.98 \pm 1.77 \mathrm{~ms}$ for control, $p=0.1439$ ), as well as pool sizes, slow time constant and rate of sustained release, were not significantly different. Thus, the small difference in the fast time constant between Munc13-1 $1^{-/}$Munc13-2 $2^{+/-}$cells and the control group (Fig. 3.5G) is most likely not due to Munc13 genotype. 


\section{6 ubMunc13-2 is Required for LDCV Release Triggered by Depolarization}

ubMunc13-2 contributes to a large extent to the priming of LDCVs for exocytosis using flash photolysis experiments, which involved a step increase in $\left[\mathrm{Ca}^{2+}\right]$ to tens of micromolar levels. In order to confirm its role in LDCV exocytosis using a more physiological stimulus, a depolarisation train consisting of six $10 \mathrm{~ms}$ depolarisations followed by four $100 \mathrm{~ms}$ depolarisations to $0 \mathrm{mV}$ was applied to chromaffin cells from P0 Munc13-2-/animals and littermate Munc13-2 $2^{+/+}$animals to test for release deficits (Fig. 3.6). The first six short depolarisations and the longer four depolarisations are thought to deplete the IRP and the RRP/fast burst, respectively (Schonn et al., 2010; Voets et al., 1999). The averaged [Ca $\left.{ }^{2+}\right]$ of Munc13-2 $2^{+/+}(n=63)$ and Munc13-2-/- $(n=60)$ cells before, during and after the depolarisation train were not significantly different (Fig. 3.6A). Ionic charge was calculated by integrating the current recorded during each depolarisation (Supplementary Fig. 3A). The currents for the $3^{\text {rd }}, 4^{\text {th }}, 5^{\text {th }}$ and $6^{\text {th }}$ depolarisations (all $10 \mathrm{~ms}$ long) were slightly smaller in Munc13-2 $2^{-/-}$than in Munc13-2 $2^{+/+}$cells $(p<0.05)$. The average amplitude of the ionic current measured during the steady state of the first $100 \mathrm{~ms}$ depolarisation $(2.239 \mathrm{~s}$ to $2.249 \mathrm{~s}$ after the start of recording) was not significantly different between the two groups. Thus, the amount of $\mathrm{Ca}^{2+}$ entry during each depolarisation was most likely similar in the Munc13-2 $2^{+/+}$ and Munc13-2 ${ }^{-/-}$cells. Capacitance measurements showed a release deficit in Munc13-2 $2^{-/}$ cells (Fig. 3.6A). The capacitance increases $(\Delta \mathrm{Cm})$ after the $1^{\text {st }}(p<0.001), 2^{\text {nd }}(p<0.001)$, $4^{\text {th }}(p<0.001), 5^{\text {th }}(p<0.01)$, and $6^{\text {th }}(p<0.001) 10 \mathrm{~ms}$ depolarisations, as well as the $1^{\text {st }}(\mathrm{p}<$ $0.05), 2^{\text {nd }}$ to $4^{\text {th }}(p<0.001) 100 \mathrm{~ms}$ depolarisations in Munc13-2 $2^{-/-}$cells were significantly smaller compared to Munc13-2-/- cells (Fig. 3.6B). Accordingly, the total $\Delta \mathrm{Cm}$ after the train is significantly diminished in Munc13-2 $2^{-/-}$cells $(124.87 \pm 10.48 \mathrm{fF})$ compared to that in Munc13-2 $2^{+/+}$cells $(314.25 \pm 16.05 \mathrm{fF}, p<0.001)$. Thus, deletion of Munc13-2 significantly reduces the number of LDCVs that can be released in response to $\mathrm{Ca}^{2+}$ influx through $\mathrm{Ca}^{2+}$ channels, namely the IRP as well as the RRP. 

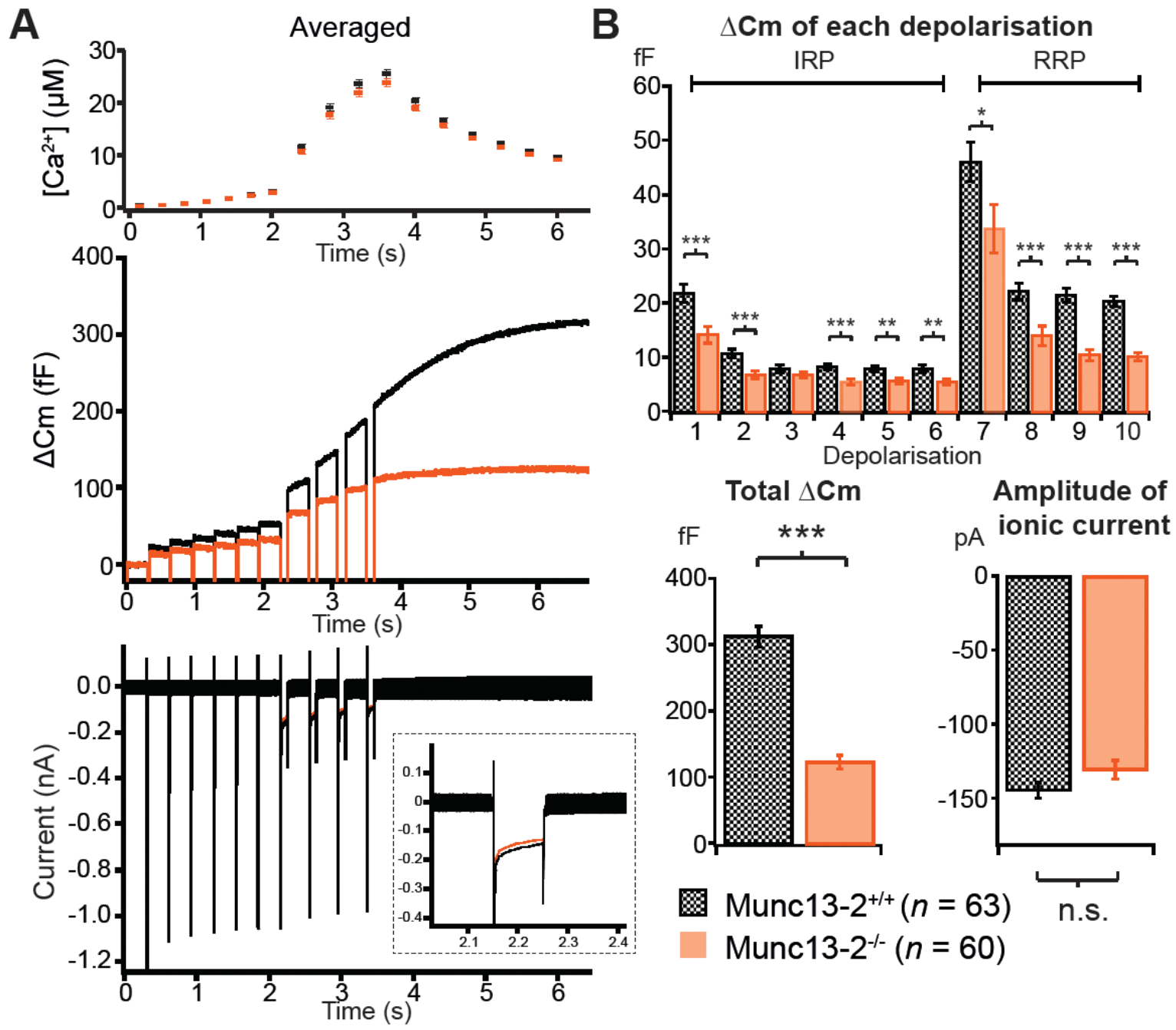

Fig. 3.6 Depolarisation of Munc13-2 ${ }^{-/-}(n=60)$ chromaffin cells with littermate Munc13-2 $2^{+/+}$ $(n=63)$ cells as control. (A) Averaged $\left[\mathrm{Ca}^{2+}\right]$, capacitance traces and current traces during $6 \times 10 \mathrm{~ms}$ and $4 \times 100 \mathrm{~ms}$ depolarisations to $0 \mathrm{mV}$. The inset next to the current traces showed the first $100 \mathrm{~ms}$ depolarisation. (B) $\Delta \mathrm{Cm}$ after each depolarisation, total $\Delta \mathrm{Cm}$ and average amplitudes of the ionic currents from $2.239 \mathrm{~s}$ to $2.249 \mathrm{~s}$ after the start of recording for the two groups $(* * *, p<0.001 ; * *, \mathrm{p}<0.01, *, p<0.05)$.

\section{7 ubMunc13-2 and Munc13-1 Differentially Rescue LDCV Exocytosis in Munc13- 1/2 DKO Chromaffin Cells}

To compare the function of ubMunc13-2 and Munc13-1 in the DKO background, rescue experiments were performed with both isoforms. First, over-expression of ubMunc13$2(n=36)$ in Munc13-1/2 DKO cells using Semliki Forest virus was compared with overexpression of EGFP $(n=32)$ as control (Fig. 3.7). Overexpression of ubMunc13-2 dramatically enhanced release from Munc13-1/2 DKO cells, apparently to levels much higher 
than in WT cells (Fig. 3.7A-C). In virus-infected cells, using the same $\left[\mathrm{Ca}^{2+}\right]$ measurement protocol as in experiments with uninfected cells, $\left[\mathrm{Ca}^{2+}\right]$ after the flash decayed much more slowly (Fig. 3.7C), which could lead to an increase in the sustained rate of release. In addition, post-flash $\left[\mathrm{Ca}^{2+}\right]$ of EGFP-expressing cells measured at three time points during the sustained phase of release was significantly higher than in ubMunc13-2-overexpressing cells $(p<0.05)$. However, this difference can probably be discounted as not having a significant effect on experimental outcome, since the amount of exocytosis in ubMunc13-2overexpressing cells drastically exceeded that in EGFP-overexpressing cells. Virus infection also affected the properties of the cells such that traces obtained from cells with small pool sizes, e.g. EGFP-expressing Munc13-1/2 DKO cells, became difficult to analyse by a sum of two exponentials (Fig. 3.7E). Thus, only a subset of cells was analysed using two exponential fitting. The averaged fast burst in ubMunc13-2-overexpressing cells was $314.24 \pm 79.59 \mathrm{fF}$ ( $n$ $=22)$, which was much larger than that in control cells $(12.38 \pm 4.91 \mathrm{fF}, n=6)$, but the difference was statistically not significant due to the small number of traces from EGFP expressing cells that could be fitted. The slow burst (ubMunc13-2 cells, $580.53 \pm 76.21 \mathrm{fF}$; EGFP cells, $25.05 \pm 8.12 \mathrm{fF}, p<0.001$ ) and the rate of sustained release (ubMunc13-2 cells, $396.49 \pm 31.80 \mathrm{fF} / \mathrm{s}$; EGFP cells, $4.00 \pm 2.29 \mathrm{fF} / \mathrm{s}, p<0.001$ ) were significantly enhanced in ubMunc13-2-overexpressing cells. The fast time constant was not altered (ubMunc13-2 cells, $23.74 \pm 3.00 \mathrm{~ms}$; EGFP cells, $22.95 \pm 8.61 \mathrm{~ms}$ ), but the slow time constant was longer in ubMunc13-2-overexpressing cells than in control cells (ubMunc13-2 cells, 653.19 \pm 124.30 $\mathrm{ms}$; EGFP cells, $189.41 \pm 33.28 \mathrm{~ms}$ ), although the difference is not significant. The slower release of ubMunc13-2 within the first second after the flash was also evident in the overlaid normalised traces (Fig. 3.7F). Since not all the traces acquired could be fitted with a sum of exponentials, the average of all traces from each group (Fig. 3.7C, ubMunc13-2, $n=36$; EGFP, $n=32$ ) was fitted with a sum of two exponentials, and the results are shown in Fig. 3.7G. The parameters obtained showed a similar trend as in the two exponential fitting analysis of individual traces. In summary, ubMunc13-2 rescued the release deficit in Munc13-1/2 DKO cells, confirming that the defective release in these cells is due to the absence of ubMunc13-2. In the presence of large amounts of ubMunc13-2, the slow time constant of release is longer. 


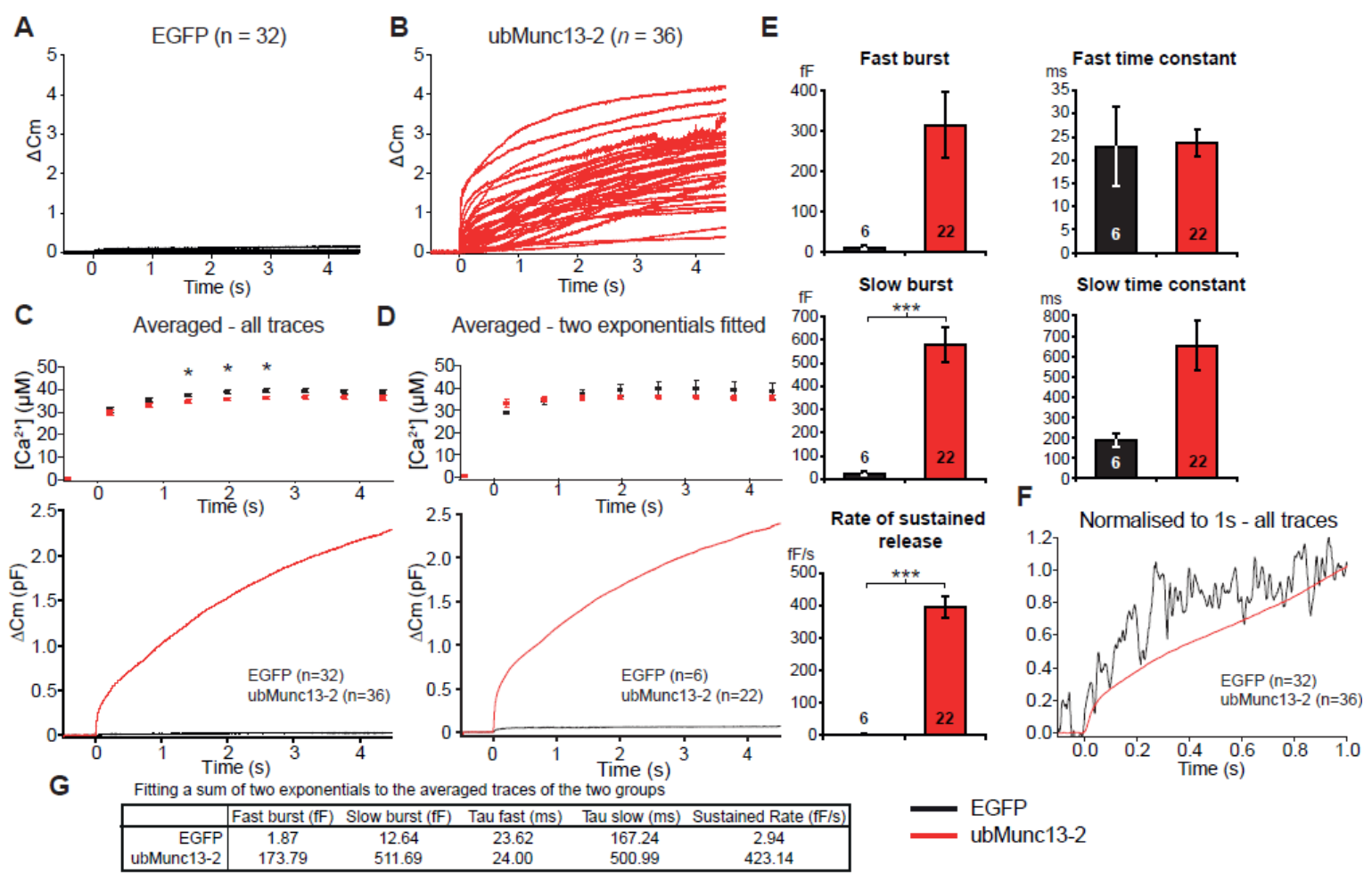

Fig. 3.7 Rescue of defective release in Munc13-1/2 DKO cells by viral overexpression of ubMunc13-2. Capacitance traces from (A) EGFP-overexpressing control cells $(n=32)$, (B) ubMunc13-2 expressing cells $(n=36)$. (C) Averaged $\left[\mathrm{Ca}^{2+}\right]$ and capacitance traces from the two groups. (D) Averaged $\left[\mathrm{Ca}^{2+}\right]$ and capacitance traces from cells that can be fitted by a sum of two exponentials (ubMunc13-2 cells, $n=22$; EGFP cells, $n=6$ ). (E) Sizes of the fast burst, slow burst, their time constants and the rate of sustained release from the two groups $(* * *, p<0.001)$. (F) Capacitance traces normalised at 1s. (G) Pool sizes and time constants obtained by fitting a sum of two exponentials to the averaged traces shown in (C).

Previous studies have reported that overexpression of ubMunc13-2 in WT bovine chromaffin cells results in faster vesicle replenishment than that of Munc13-1 (Ashery et al., 2000; Zikich et al., 2008). In addition, in hippocampal neurons, Munc13-1 expression causes depression in response to $10 \mathrm{~Hz}$ stimulation, while ubMunc13-2 expression causes facilitation (Junge et al., 2004; Rosenmund et al., 2002). In order to examine the inherent differences in priming of LDCVs in a cleaner background devoid of interference of possibly competing Munc13 isoforms, rescue of Munc13-1 was performed by viral overexpression in Munc13-1/2 DKO cells (Fig. 3.8). Statistical analysis of parameters from exponential fittings was not performed in this experiment due to insufficient cell numbers. Munc13-1 overexpression $(n=17)$ was compared to EGFP expression $(n=13)$ and also rescued the release deficit in Munc13-1/2 DKO cells (Fig. 3.8A-C). When averaged traces of ubMunc132-overexpressing and Munc13-1-overexpressing cells were overlaid, it was observed that 
ubMunc13-2 expression caused a larger exocytotic burst and faster sustained release than Munc13-1 (Fig. 3.8D). Traces normalised at $1 \mathrm{~s}$ showed that, in contrast to rescue by ubMunc13-2, rescue by Munc13-1 did not demonstrate slower release within the first second after the flash (Fig. 3.8E). This suggests that, although the release deficit can be rescued by either isoforms, Munc13-1 and Munc13-2 have distinct properties in chromaffin cells that differentially affect the efficiency of LDCV recruitment for release, especially the rate of maturation of vesicles during ongoing stimulation when $\left[\mathrm{Ca}^{2+}\right]$ is high.
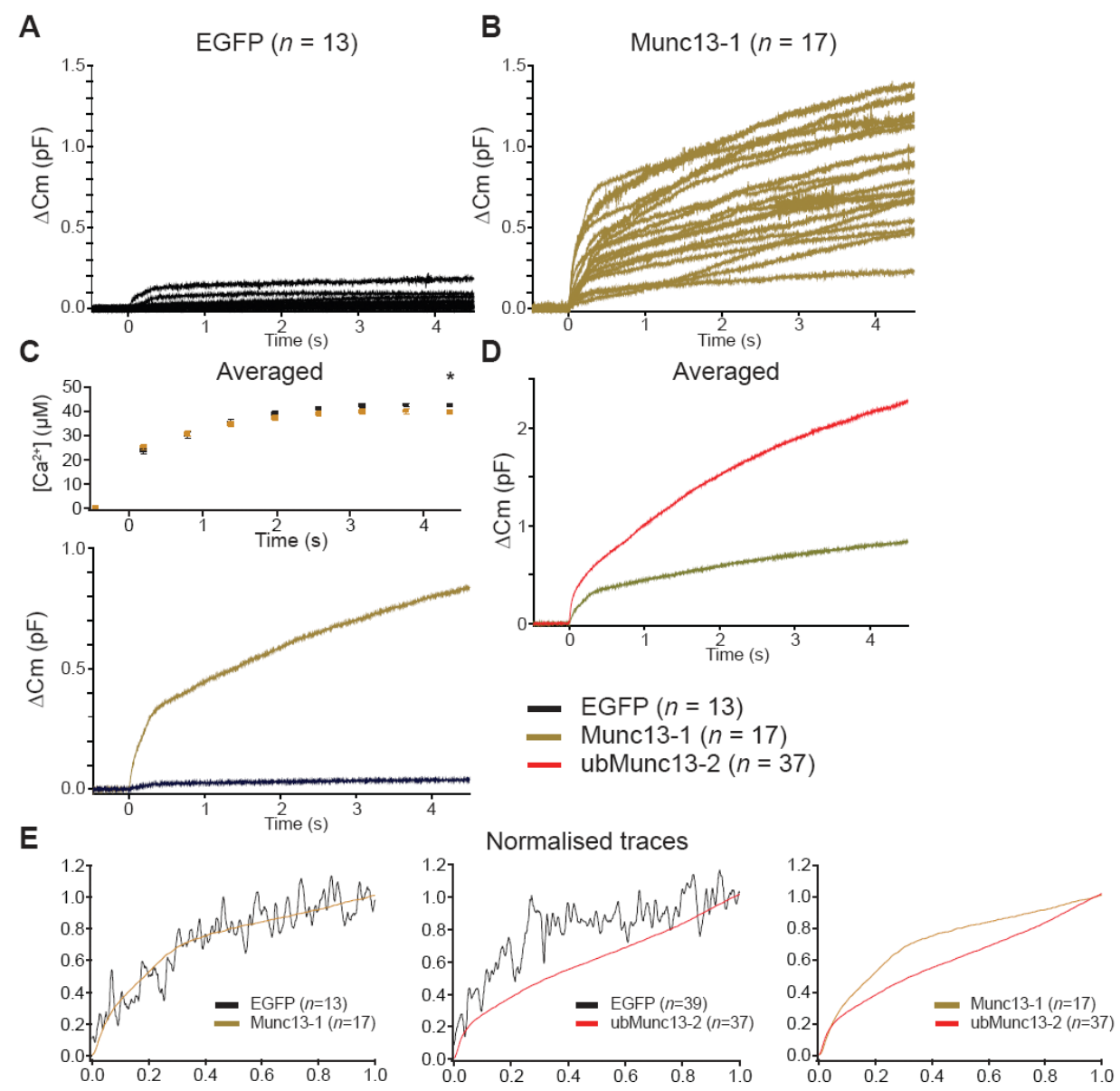

Fig. 3.8 Rescue of Munc13-1/2 DKO chromaffin cells with viral Munc13-1 overexpression. Traces from (A) EGFP-overexpressing cells $(n=13)$, (B) Munc13-1-overexpressing cells ( $n$ = 17). (C) Averaged $\left[\mathrm{Ca}^{2+}\right]$ and capacitance traces from the two groups. (D) Overlay of capacitance traces in rescue experiments by ubMunc13-2 and Munc13-1 overexpression. (E) Traces normalised at $1 \mathrm{~s}$ after the flash for EGFP-overexpressing and Munc13-1overexpressing cells (left panel), EGFP-overexpressing cells and ubMunc13-2overexpressing cells in the rescue using ubMunc13-2 expression (centre), and a comparison of ubMunc13-2-overexpressing and Munc13-1-overexpressing cells (right panel). 


\subsection{Baiap3 is Not Required for LDCV Exocytosis in Chromaffin Cells}

ubMunc13-2 has a dominant role in priming LDCVs for exocytosis in chromaffin cells. Relatively small contributions by other isoforms, like the involvement of Munc13-1, could be masked in its presence. To test if Baiap3 plays role in LDCV exocytosis in the absence of Munc13-1 and Munc13-2, viral overexpression of Baiap3 in Munc13-1/2 DKO cells was performed (Fig. 3.9). A comparison of averaged traces showed that Baiap3 does not lead to a significant rescue of the release deficit in Munc13-1/2 DKO cells (Fig. 3.9C). The capacitance traces of both Baiap3-overexpressing $(n=33)$ and EGFP-overexpressing $(n=28)$ cells had small amplitudes, precluding analysis by fitting of sums of exponentials. Hence, the $\Delta \mathrm{Cm}$ that occurred within $0.5 \mathrm{~s}$ and between $0.5 \mathrm{~s}$ to $4 \mathrm{~s}$ after the flash were measured to assess the size of the exocytotic burst and sustained release, respectively (Fig. 3.9D). The exocytotic burst and rate of sustained release of Baiap3-overexpressing and control cells were not significantly different from each other. Thus, Baiap3 does not rescue the defective release in Munc13-1/2 DKO cells and therefore does not appear to function in priming LDCVs for release.
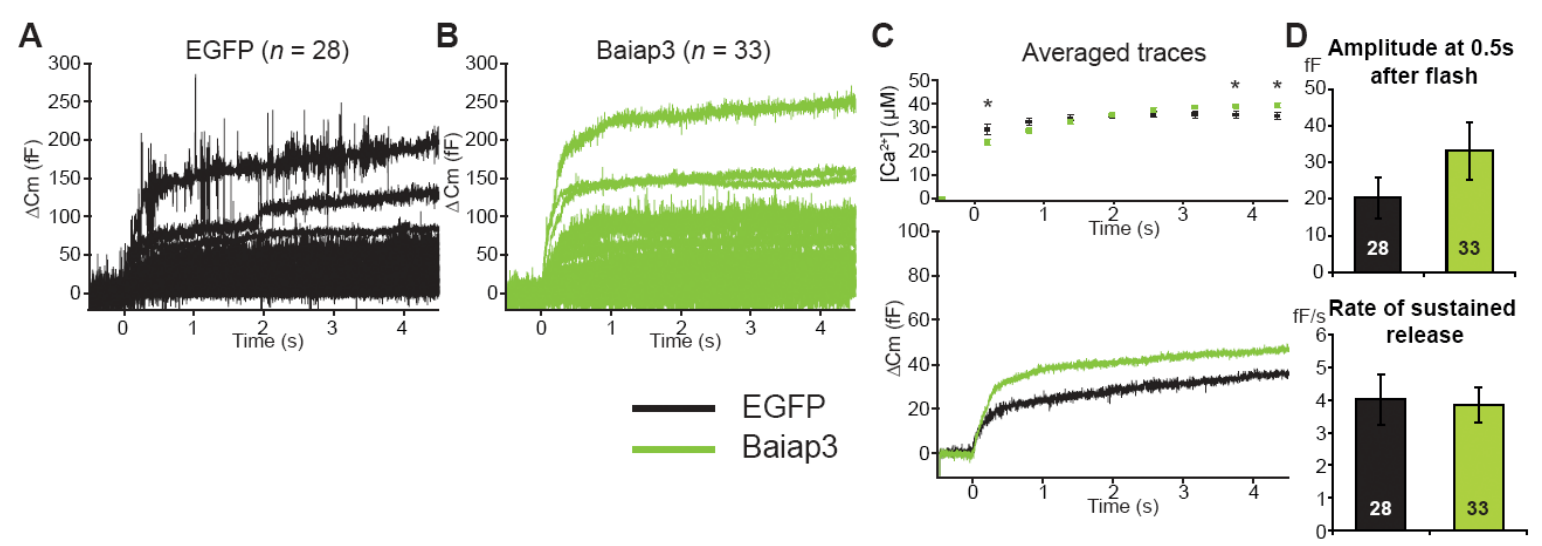

Fig. 3.9 Baiap3 overexpression in Munc13-1/2 DKO chromaffin cells. Traces from (A) EGFP-overexpressing control cells $(n=28)$, and (B) Baiap3-overexpressing cells $(n=33)$. (C) Averaged $\left[\mathrm{Ca}^{2+}\right]$ and capacitance traces from the two groups. (D) $\Delta \mathrm{Cm}$ within the first $0.5 \mathrm{~s}$ after the flash to indicate the size of exocytotic burst and $\Delta \mathrm{Cm}$ from $0.5 \mathrm{~s}$ to $4 \mathrm{~s}$ after the flash to indicate sustained release.

To test if Baiap3 has any role in tethering LDCVs in the proximity of $\mathrm{Ca}^{2+}$ channels, depolarisation trains were applied ( $6 \times 10 \mathrm{~ms}$ and $4 \times 100 \mathrm{~ms}$ to $0 \mathrm{mV}$, as before) to Baiap3 $\mathrm{KO}$ cells to elicit release of IRP vesicles. Averaged $\left[\mathrm{Ca}^{2+}\right]$, capacitance and current traces of Baiap $^{+/+}(n=116)$ and Baiap3 ${ }^{-/-}(n=103)$ cells are shown in Fig.3.10A. The total $\Delta \mathrm{Cm}$ after the train was significantly increased in Baiap $3^{-/-}$cells $(269.03 \pm 10.82 \mathrm{fF})$ compared to 


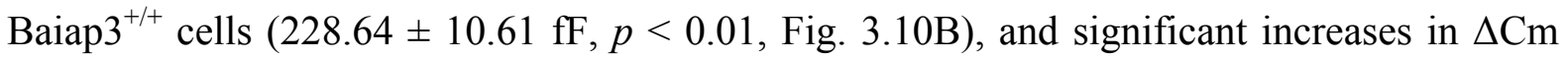
was observed in Baiap3 $3^{-/}$cells for the $6^{\text {th }} 10 \mathrm{~ms}$ depolarisation and the $3^{\text {rd }} 100 \mathrm{~ms}$ depolarisation (both $p<0.05$, Fig. 3.10B). However, the integrated ionic current during individual depolarisations ( $p<0.05$, Supplementary Fig. 3B), as well as the averaged amplitude of ionic current ( $p<0.05$, Fig. 3.10B) were found to be mildly but significantly increased in Baiap $3^{-/-}$cells. To rule out the effect of increased $\mathrm{Ca}^{2+}$ influx on priming, Baiap $^{+/+}$and Baiap3 $3^{-/-}$cells were matched for equal current during the first $100 \mathrm{~ms}$ depolarisation (Fig. 3.11). After matching, current charge of each depolarisation and the average amplitude of ionic current were not significantly different between the two groups (Supplementary Fig. 3C, Fig. 3.11B), and no differences were observed in the total $\Delta \mathrm{Cm}$ and $\Delta \mathrm{Cm}$ after each depolarisation (Fig. 3.11A, B). Thus, there is no evidence that Baiap3 affects the size of the IRP and Baiap3 therefore does not appear to function in localizing LDCVs to $\mathrm{Ca}^{2+}$ channels.

A
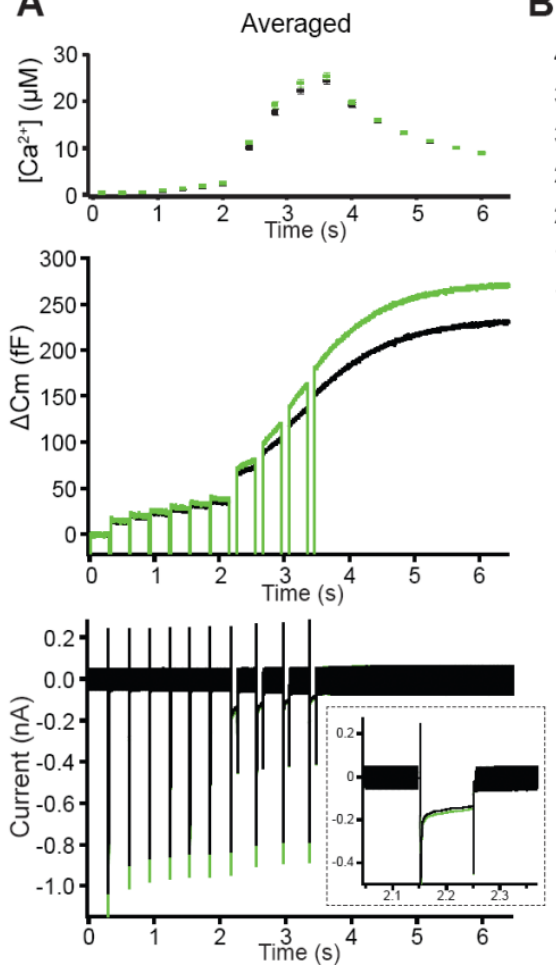

B
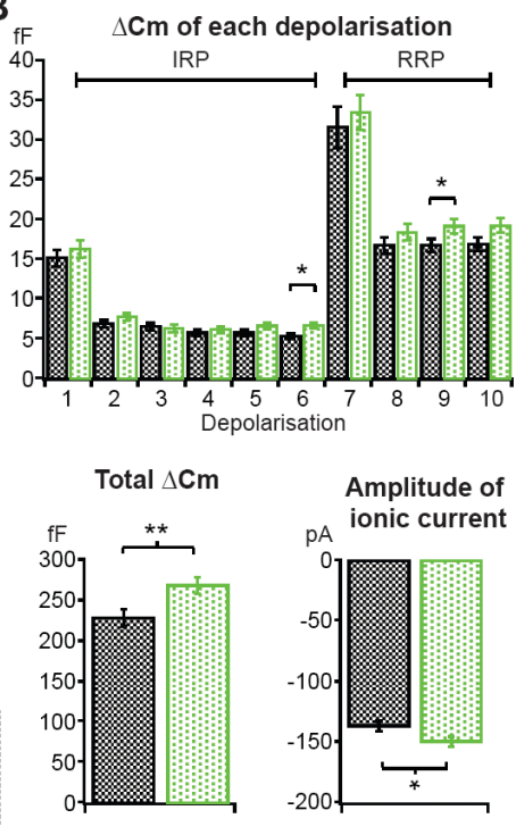

$\mathrm{pA}$ ionic current

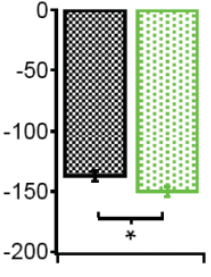

Baiap $3^{+/+}(n=116)$

Baiap3 $\%(n=103)$

Fig. 3.10 Depolarisation stimulation of Baiap3 $3^{-/-}(n=103)$ and littermate Baiap $3^{+/+}(n=$ 116) cells. (A) Averaged $\left[\mathrm{Ca}^{2+}\right]$, capacitance and current traces for the two groups. Inset next to the current trace showed the first $100 \mathrm{~ms}$ depolarisation. (B) $\Delta \mathrm{Cm}$ after each depolarisation, total $\Delta \mathrm{Cm}$, and average amplitude of ionic current between $2.239 \mathrm{~s}$ and 2.249 $\mathrm{s}$ after the start of recording $(* *, p<0.01 ; *, p<0.05)$. 


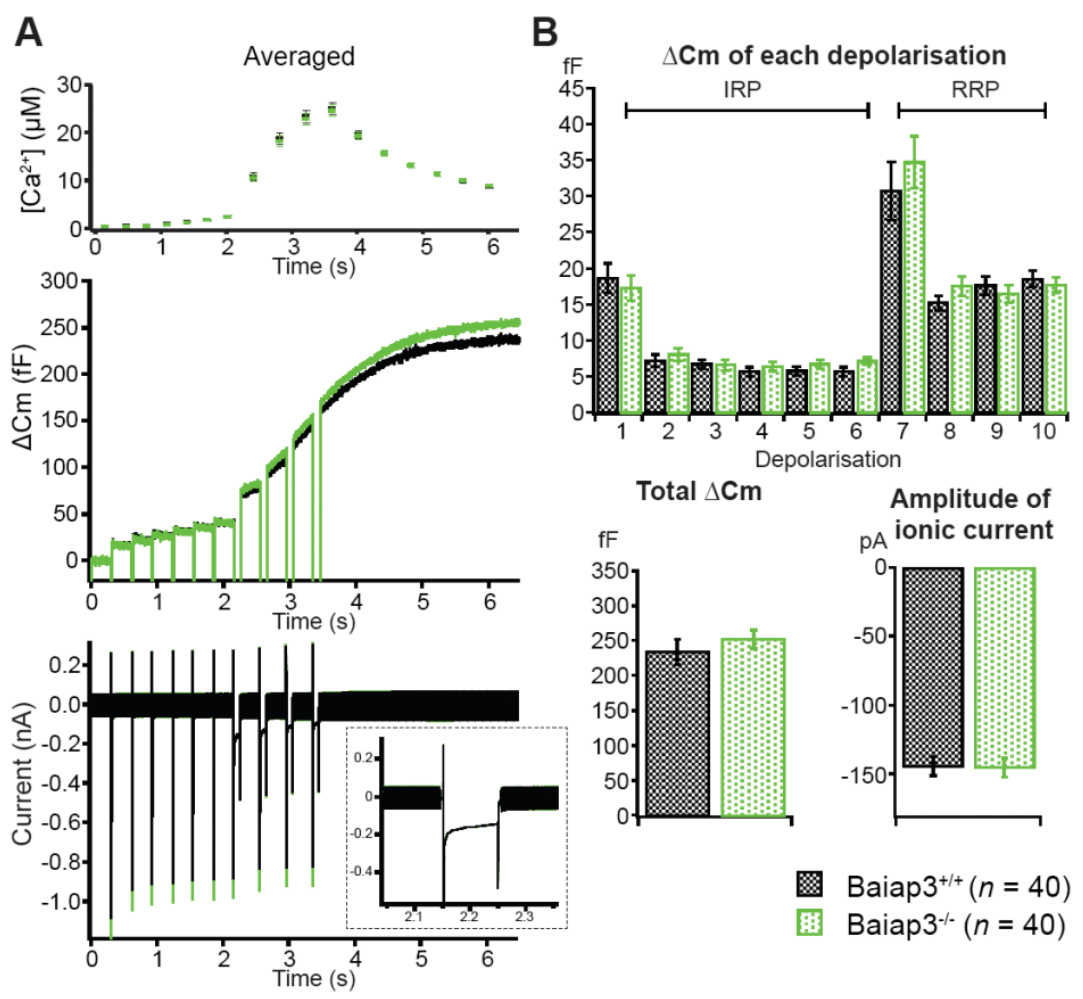

Fig. 3.11 Depolarisation stimulation of Baiap3 $3^{-/-}(n=40)$ and littermate Baiap3 ${ }^{+/+}(n=40)$ cells after matching for equal current influx. (A) Averaged $\left[\mathrm{Ca}^{2+}\right]$, capacitance and current traces for the two groups. Inset next to the current trace showed the first $100 \mathrm{~ms}$ depolarisation. (B) $\Delta \mathrm{Cm}$ after each depolarisation, total $\Delta \mathrm{Cm}$ and average amplitude of ionic current between $2.239 \mathrm{~s}$ and $2.249 \mathrm{~s}$ after the start of recording.

Lastly, to probe whether Baiap3 might negatively affect LDCV exocytosis in a WT context, Baiap3 was overexpressed in WT mouse chromaffin cells. Traces from EGFPoverexpressing $(n=34)$ and Baiap3-overexpressing $(n=37)$ WT cells are shown in Fig. 3.12. An overlay of averaged traces showed that LDCV exocytosis was very similar in both experimental groups. Fitting by a sum of two exponentials was possible for a subset of cells (EGFP-overexpressing, $n=27$, Baiap3-overexpressing, $n=25$ ). This analysis revealed that the sizes of the fast burst $(58.57 \pm 13.02 \mathrm{fF}$ for EGFP-overexpressing cells; $44.34 \pm 11.89 \mathrm{fF}$ for Baiap3-overexpressing cells), slow burst (90.90 $\pm 9.14 \mathrm{fF}$ for EGFP-overexpressing cells; $126.35 \pm 28.89 \mathrm{fF}$ for Baiap3-overexpressing cells) and their time constants (fast time constant: $28.12 \pm 2.68 \mathrm{~ms}$ for EGFP-overexpressing cells, $25.93 \pm 3.11 \mathrm{~ms}$ for Baiap3overexpressing cells; slow time constant: $286.55 \pm 27.55 \mathrm{~ms}$ for EGFP-overexpressing cells, $234.39 \pm 14.13 \mathrm{~ms}$ for Baiap3-overexpressing cells), as well as the rate of sustained release $(47.19 \pm 4.73 \mathrm{fF} / \mathrm{s}$ for EGFP-overexpressing cells; $52.20 \pm 6.17 \mathrm{fF} / \mathrm{s}$ for Baiap3overexpressing cells) were not significantly different from each other. Thus, Baiap3 appears 
to have a function in chromaffin cells that is distinct from the regulation of LDCV exocytosis.
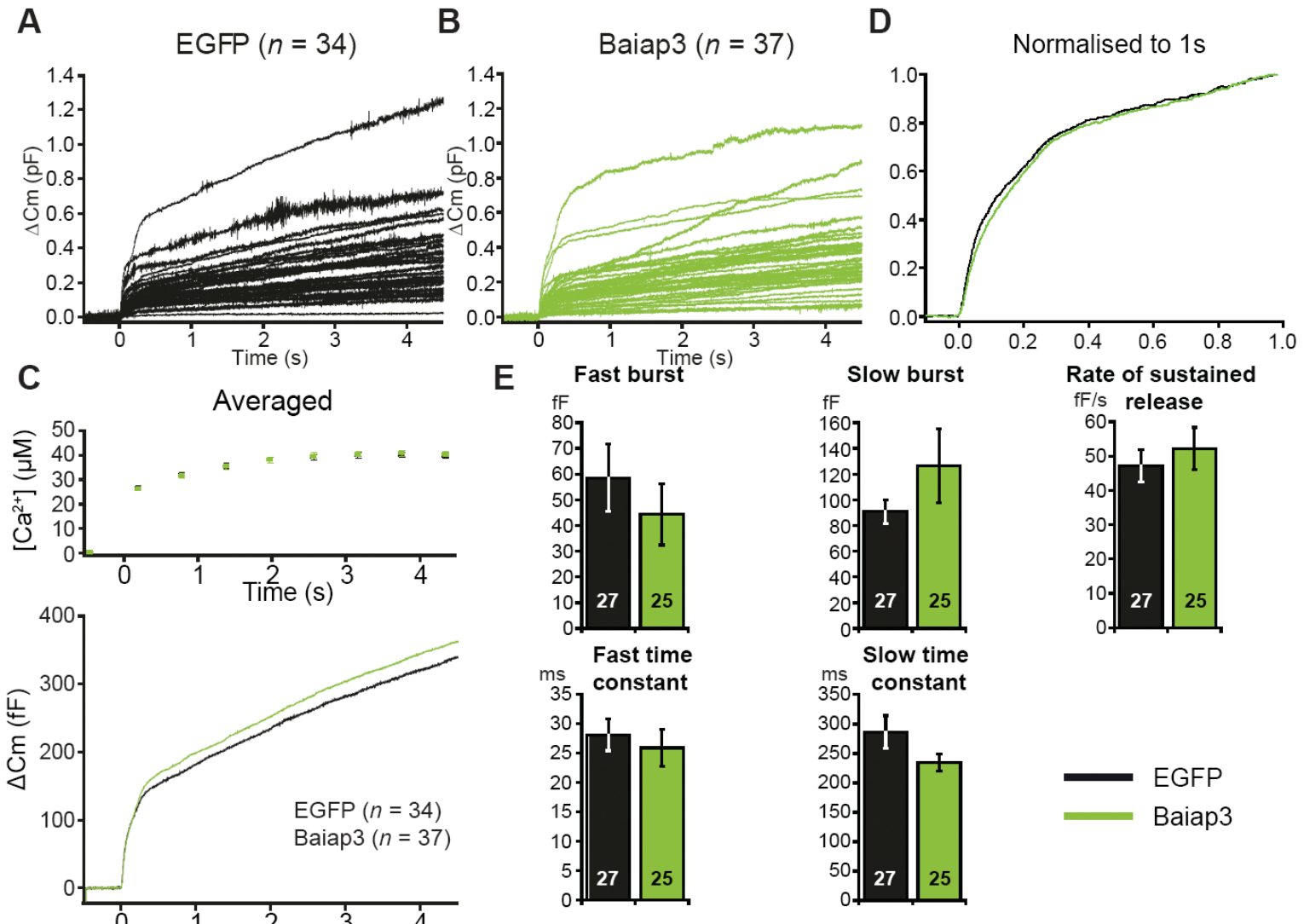

Rate of sustained
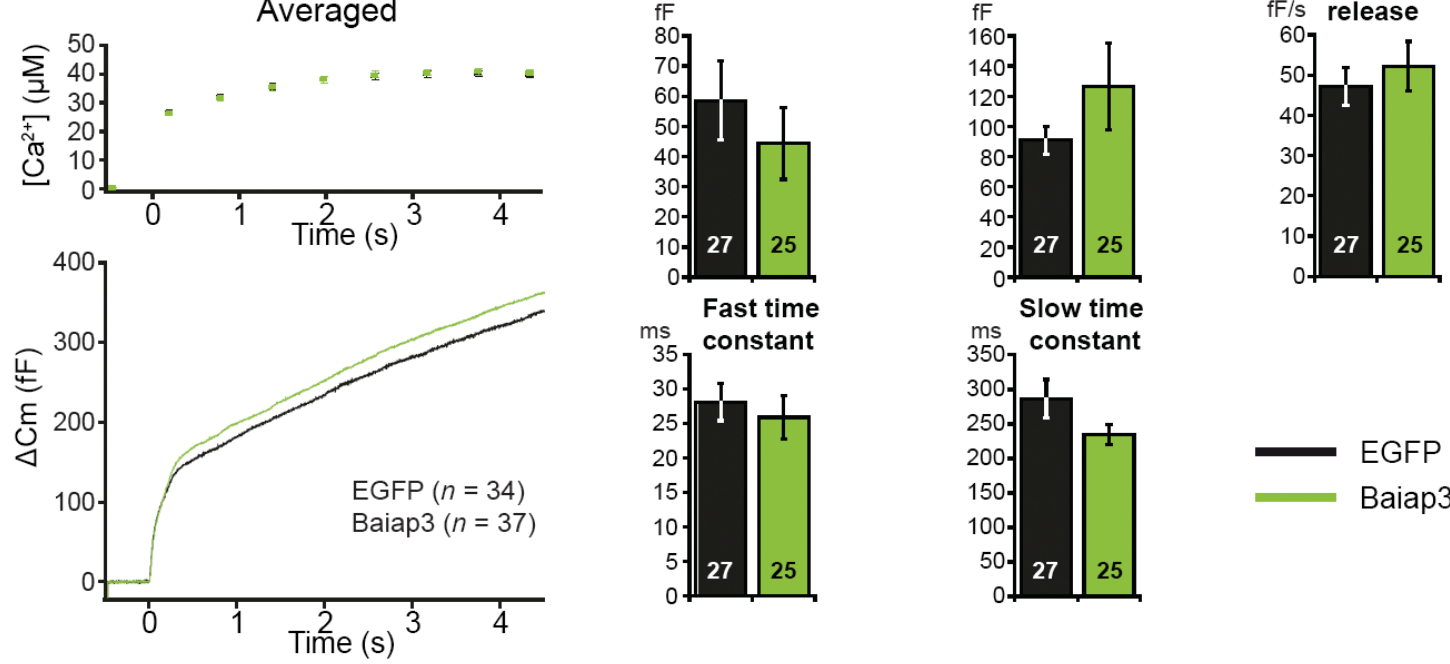

Fig. 3.12 LDCV exocytosis as measured by capacitance changes during flash photolysis of caged $\mathrm{Ca}^{2+}$ in P0 WT chromaffin cells overexpressing either EGFP $(n=34)$ or Baiap3 $(n=$ 37). Traces from (A) EGFP-overexpressing cells, (B) littermate Baiap3-overexpressing cells. (C) Averaged $\left[\mathrm{Ca}^{2+}\right]$ and capacitance traces for both groups before and after the flash. (D) Normalised traces within the first second after the flash. (E) Sizes and time constants of the two components of exocytotic burst and rate of sustained release. 


\section{Discussion}

This study is the first to demonstrate a deficit in chromaffin cell LDCV exocytosis in the absence of Munc13 isoforms. LDCV exocytosis was most strongly impaired in the absence of ubMunc13-2, while the contribution of endogenous Munc13-1 to LDCV exocytosis only became apparent in the absence of Munc13-2. Single deletions of other Munc13 isoforms, namely Munc13-3 and Baiap3, did not affect LDCV exocytosis in mouse chromaffin cells.

The comparative analysis of LDCV exocytosis in genetic deletion mutants of the various Munc13 isoforms enabled me to show that Munc13s play an essential role in priming LDCVs for both fast and ongoing release in chromaffin cells. Major findings of this study are that ubMunc13-2 is the dominant isoform involved in priming LDCVs into releasable pools in chromaffin cells, and that Munc13-1 is involved in priming vesicles into the SRP and in the maturation of LDCVs for release during ongoing stimulation (Fig. 3.5). ubMunc13-2 alone accounts for $\approx 60 \%$ of release from the RRP, $\approx 52 \%$ of release from the SRP and $72 \%$ of ongoing sustained release. By contrast, a role for Baiap3, a Munc13 isoform with prominent expression in the adrenal medulla, was not evident in my analysis of LDCV exocytosis. Although the presence of a functional truncated Baiap3 product in perinatal adrenal glands of Baiap3 KO mice cannot be ruled out (Fig. 3.1D, Supplementary Fig. 1), the inability of Baiap3 to rescue the release deficit of Munc13-1/2 DKO cells suggests that it likely does not function in regulating LDCV exocytosis in chromaffin cells (Fig. 3.9). Since bMunc13-2, Munc13-3 and Munc13-4 are not detectable in perinatal adrenal glands (Fig. 3.1B, C, E), and the abrogation of Munc13-3 caused no significant difference in LDCV exocytosis from chromaffin cells (Fig. 3.4), I concluded that the majority of priming in chromaffin cells into different kinetic pools is mediated by ubMunc13-2, while Munc13-1 plays a minor role in mediating release, particularly from the SRP and sustained component.

\subsection{Contributions of Munc13 Isoforms to LDCV Exocytosis in Chromaffin Cells}

The contributions of Munc13 isoforms in LDCV exocytosis in chromaffin cells largely correlate with their expression levels in adrenal glands, except for Baiap3. ubMunc13-2 and Baiap3 are the most abundant isoforms expressed in the adrenal gland (Fig.

3.1B, D, F, G, H), while Munc13-1 expression is low (Fig. 3.1A, F, G, H). ubMunc13-2 is 
largely responsible for priming LDCVs into releasable pools, while Munc13-1 appears to be dispensable in a WT genetic background, but its function became apparent upon Munc13-2 deletion (Fig. 3.5). ubMunc13-2 is important in maintaining normal secretion levels triggered by $\mathrm{Ca}^{2+}$ influx, as shown by a marked reduction of release from the IRP and RRP triggered by depolarisation trains (Fig. 3.6). bMunc13-2 is not expected to contribute to LDCV secretion in chromaffin cells, as its expression cannot be detected in adrenal glands (Fig. 3.1B). A role of Munc13-3 in LDCV secretion from chromaffin cells is not likely, as its expression is undetectable in adrenal glands and flash photolysis experiments showed no obvious alterations of secretion from Munc13-3 KO animals (Fig. 3.1C, 3.4). However, since the effect of Munc13-1 only became apparent in the absence of Munc13-2, the deletion of Munc13-3 in the background of the Munc13-1/2 DKO may be required to unequivocally rule out an involvement of Munc13-3 in LDCV exocytosis in mouse chromaffin cells.

Overexpression of ubMunc13-2 results in a longer slow time constant in Munc13-1/2 DKO cells (Fig. 3.7) and in WT cells (Zikich et al., 2008). In the PPM (see section "Vesicle Pool Dynamics in Chromaffin Cells"), if post-flash $\left[\mathrm{Ca}^{2+}\right]$ are similar between control cells and ubMunc13-2-overexpressing cells, this would mean that ubMunc13-2 overexpression interferes with downstream $\mathrm{Ca}^{2+}$-binding steps of the SRP. How an increase in Munc13 levels would be able to slow down $\mathrm{Ca}^{2+}$-binding steps is currently unknown.

\subsubsection{Contributions of Munc13-1 to LDCV Exocytosis in Chromaffin Cells}

Abrogation of Munc13-1 alone did not have a major impact on the size of any component of release (Fig. 3.2). In the presence of Munc13-2 its contribution to priming into the RRP, SRP and for ongoing release appears minor compared to that of ubMunc13-2. Compared to Munc13-1 $1^{+/}$Munc13-2 $2^{+/-}$and Munc13-1 $1^{+/-}$Munc13-2 $2^{+/-}$control cells Munc13-1 contributes maximally to only $\approx 11 \%$ of priming into the RRP, $\approx 14 \%$ priming into the SRP and $\approx 15 \%$ of priming for sustained release (\% of control levels, Fig. 3.5G). This difference can most simply be explained by the much higher expression level in chromaffin cells of ubMunc13-2 compared to Munc13-1 (Fig. 3.1A, B, F, G). However, in the absence of ubMunc13-2, when setting the release in Munc13-1 $1^{+/+}$Munc13-2/- cells to $100 \%$, it contributes to $\approx 30 \%$ of the remaining slow burst release and $\approx 55 \%$ of the remaining sustained release (Table 3.2). Thus, in the absence of Munc13-2, Munc13-1 contributes significantly to residual release. 
In contrast to the effect of Munc13-1 observed in the absence of Munc13-2, no reductions in RRP and SRP pool sizes and in sustained release were observed in Munc13-1 single KO cells, but a mild increase in the SRP occurred instead $(\mathrm{p}<0.05$, Fig. 3.2). There can be two explanations for this phenomenon. First, the higher expression level of ubMunc13-2 results in preferential localisation of ubMunc13-2 at the actual release sites, such that the removal of Munc13-1 does not impact secretion to a significant degree, because most of the release sites are occupied by ubMunc13-2. However, differential localization of ubMunc13-2 and Munc13-1 in chromaffin cells has not been shown to date. An alternative explanation would thus be that, release site occupancy may not be strongly biased in favour of one isoform, but since ubMunc13-2 appears to be more efficient in priming LDCVs in chromaffin cells than Munc13-1 (Fig. 3.8), removing Munc13-1 does not affect release significantly. Most likely, the differences in expression levels and priming efficiency both contribute to the observed effects. Although a direct comparison with WT cells was not made experimentally, overexpression of Munc13-1 is able to rescue Munc13-1/2 DKO secretion to WT levels (exocytotic burst size in the range of 300-400 fF, Fig. 3.8). However, endogenous Munc13-1 in the absence of Munc13-2 is not sufficient to support release to levels similar to that of WT (Fig. 3.5). Thus, the low endogenous expression levels of Munc13-1 probably contribute to a larger degree than its lower priming efficiency to the release deficit in Munc13-1 ${ }^{+/+}$Munc13-2/- cells. The ability of Munc13-1 overexpression to rescue the release deficit of the Munc13-1/2 DKO indicates that Munc13-1 is able to use the release sites originally occupied by ubMunc13-2 and is thus a further argument against the existence of differential occupation of release sites by ubMunc13-2 and Munc13-1.

The apparent increase in the SRP in Munc13-1/- cells compared to Munc13-1 ${ }^{+/+}$cells (Fig. 3.2E) is difficult to explain. One possible explanation would be, that this reflects the occupancy of release sites by the more efficiently priming ubMunc13-2 isoform when Munc13-1 is absent, which would again suggest that the two isoforms compete for occupancy of release sites (Fig. 3.2E). The effect is however not evident in the comparison between Munc13-1 ${ }^{+/+}$Munc13-2 $2^{+/-}$Munc13-1 ${ }^{+/-}$Munc13-2 ${ }^{+/-}$control cells and Munc13-1 ${ }^{-/-}$Munc13-2 $2^{+/-}$ cells (Fig. $3.5 \mathrm{G}$ ), possibly because in the context of the Munc13-2 $2^{+/-}$background only around half of the ubMunc13-2 isoform compared to WT is expressed. 


\subsection{Priming by Munc13s in the Context of Vesicle Pool Models}

ubMunc13-2 is important for priming into the RRP, SRP and for sustained release (Fig. 3.5), and a significant effect of Munc13-1 on the SRP and sustained release becomes apparent in the absence of ubMunc13-2. Since ultrastructural analysis of Munc13-1/2 DKO chromaffin cells had not been completed at the time of writing, possible LDCV docking defects in chromaffin cells are not discussed here.

According to the parallel pool model (PPM) (Voets, 2000; Walter et al., 2013), the reduced sizes of the RRP and SRP in cells deficient for Munc13s can be explained by either a role of Munc13-dependent priming into the SRP only (or by stabilising the primed SRP vesicles) or by Munc13-dependent priming into both the SRP and the RRP (or by stabilising both the primed SRP and RRP). According to the PPM, vesicle recruitment from the unprimed pool to the SRP is $\mathrm{Ca}^{2+}$-dependent, and the conversion of vesicles from the SRP to the RRP is not $\mathrm{Ca}^{2+}$-dependent, at least not at pre-flash $\left[\mathrm{Ca}^{2+}\right]$ below $600 \mathrm{nM}$. By contrast, in the sequential pool model (SPM) only the RRP is capable of being released, and the SRP corresponds to the maturation of non-releasable NRP vesicles to the RRP. Since the effect of Munc13 abrogation on the sizes of the RRP and the SRP are similar (reductions to $29 \%$ and $34 \%$ of WT levels, respectively), Munc13s may act solely at the $\mathrm{Ca}^{2+}$-dependent priming step from the unprimed pool to the SRP. It is however unclear, whether Munc13s play a role in priming SRP vesicles into RRP at basal $\left[\mathrm{Ca}^{2+}\right](<600 \mathrm{nM})$ levels and whether or not this step is $\mathrm{Ca}^{2+}$-dependent. Simulations using either the PPM or the SPM, which assume $\mathrm{Ca}^{2+}$ independence and $\mathrm{Ca}^{2+}$-dependence of the (apparent) SRP to RRP conversion, respectively, can both recapitulate the recovery of the RRP after its selective depletion using depolarisation stimuli (Walter et al., 2013). The same simulation study showed that RRP recovery at high $\left[\mathrm{Ca}^{2+}\right]$ in dual $\mathrm{Ca}^{2+}$-uncaging protocols can be accounted for by the SPM, but in the PPM the conversion from the SRP to the RRP would have to be a $\mathrm{Ca}^{2+}$-dependent catalytic process. Whether or not Munc13s may function as such a $\mathrm{Ca}^{2+}$-dependent catalyst of SRP to RRP conversion remains to be seen. In the calyx of Held, recovery of the RRP from the SRP after a short depolarisation pulse which depletes the RRP is mainly dependent on the integrity of cytoskeletal transport processes but not on calmodulin or activation of phospholipase $\mathrm{C}$ (PLC) (Lee et al., 2012; Lee et al., 2013). $\mathrm{Ca}^{2+}$-dependent refilling of the RRP after longer depolarisations depleting both the RRP and the SRP is sensitive to calmodulin and PLC inhibition. Munc13 is a target of $\mathrm{Ca}^{2+} /$ calmodulin signalling and PLC signalling (through its $\mathrm{Ca}^{2+} /$ calmodulin binding site and $\mathrm{C} 1$ domain respectively) (Junge et al., 2004; Rhee et al., 
2002), and could thus theoretically be involved in the recruitment of RRP vesicles from the SRP only after activity dependent increases in $\left[\mathrm{Ca}^{2+}\right]$ occurs. $\mathrm{Ca}^{2+} /$ calmodulin signalling through Munc13-1 is important in the fast recovery of the RRP in the calyx (Lipstein et al., 2013). Thus, Munc13s are involved in the $\mathrm{Ca}^{2+}$-dependent refilling of the RRP in the calyx during activity-dependent increases in $\left[\mathrm{Ca}^{2+}\right]$, which occur in parallel to SRP refilling. However, there is no clear evidence that Munc13s are involved in the transition of vesicles from the SRP to the RRP under basal, i.e. low $\left[\mathrm{Ca}^{2+}\right]$ conditions. In chromaffin cells, a study by Zikich et al. (2008) showed that in contrast to an 3.8-fold increase in the RRP caused by ubMunc13-2 overexpression at high pre-flash $\left[\mathrm{Ca}^{2+}\right]$, at low pre-flash $\left[\mathrm{Ca}^{2+}\right](\approx 100 \mathrm{nM})$ overexpression of ubMunc13-2 augments release within the first $100 \mathrm{~ms}$ after the flash (regarded as the RRP) by only 30\% (106 $\pm 27 \mathrm{fF}$ in ubMunc13-2-overexpressing cells; $74 \pm$ $11 \mathrm{fF}$ for control cells). Thus, ubMunc13-2 priming into the RRP is accelerated by increased $\left[\mathrm{Ca}^{2+}\right]$, although the enhancement of RRP by ubMunc13-2 overexpression also occurs at low pre-flash $\left[\mathrm{Ca}^{2+}\right]$. Since at high basal $\left[\mathrm{Ca}^{2+}\right]$ a 5-fold increase in the SRP parallels the 3.8 -fold increase in RRP upon ubMunc13-2 overexpression (Zikich et al., 2008), this $\mathrm{Ca}^{2+}$-accelerated priming can be accounted for by a role of ubMunc13-2 in priming into the SRP, causing a downstream increase in the RRP. Since the effect of ubMunc13-2 abrogation on SRP and the RRP reduction is similar, and both rescue of Munc13-1/2 DKO by ubMunc13-2 (Fig. 3.7) and overexpression of ubMunc13-2 in WT cells drastically augment both the SRP and RRP (Ashery et al., 2000), Munc13s probably act in the priming step into the SRP. In addition, the dramatic increase in burst components and sustained release by ubMunc13-2 overexpression probably implies that Munc13s actively prime vesicles into the SRP rather than preventing vesicles from the SRP from being lost into the unprimed pool. In the PPM, sustained release, which is reduced dramatically in the Munc13-1/2 DKO, occurs through the SRP rather than the RRP because priming from the SRP to the RRP is slow and $\mathrm{Ca}^{2+}$-independent (Walter et al., 2013). By contrast, in the SPM, the effect of Munc13s on sustained release would reflect ongoing $\mathrm{Ca}^{2+}$-dependent maturation of previously non-releasable vesicles to the RRP. However, since in the SPM, the time constants and amplitudes are interdependent, detailed simulation analysis may be required to determine how well the observed effects of Munc13 deletion fit into the SPM. 


\subsection{Factors That May Mediate Residual Release in Munc13-1/2 DKO Cells}

In the absence of both ubMunc13-2 and Munc13-1, $\approx 29 \%$ of RRP, $\approx 34 \%$ of SRP and $\approx 13 \%$ of sustained release remained. This is unlike the effect of the Munc13-1/2 DKO in hippocampal neurons, where SV release was completely abolished (Varoqueaux et al., 2002). It is possible that the residual release in chromaffin cells is mediated by CAPS proteins. CAPS proteins have an established role in priming LDCVs for release (Liu et al., 2010; Liu et al., 2008; Sieburth et al., 2007; Speese et al., 2007; Zhou et al., 2007). They have been shown to bind to SNARE proteins and to accelerate SNARE-mediated liposome fusion (James et al., 2008; Khodthong et al., 2011). The interaction of CAPS with target membrane $\mathrm{PIP}_{2}$ via its pleckstrin homology domain accelerates SNARE-mediated liposome fusion (James et al., 2008). Thus, the role of CAPS as a scaffold on which SNARE complexes can assemble has been suggested as an aspect of its priming function (James and Martin, 2013). In CAPS DKO hippocampal neurons, evoked EPSC amplitude and RRP size are reduced substantially, and this reduction can be bypassed transiently by an increase in intracellular $\left[\mathrm{Ca}^{2+}\right]$ (Jockusch et al., 2007). The residual primed vesicles in CAPS DKO neurons have been attributed to the presence of Munc13s, because release in CAPS DKO neurons shows potentiation after PMA treatment, which has previously been shown to depend on Munc13s. Bypass of release reduction in CAPS DKO neurons, mediated by Munc13, required elevated intracellular $\left[\mathrm{Ca}^{2+}\right]$ for a duration of $0.5-2 \mathrm{~s}$ (Jockusch et al., 2007). Because Munc13-1/2 DKO neurons are completely silent but CAPS DKO cells can still release Munc13dependently, one hypothesis for this observation is that in hippocampal neurons, CAPS lowers the $\mathrm{Ca}^{2+}$-dependence or threshold for Munc13-mediated priming. This entails that CAPS cannot prime independently without Munc13. However, in the current study, residual priming can be observed after deletion of Munc13. One explanation for this may be intrinsic differences between chromaffin cells and hippocampal neurons. CAPS alone may be capable of priming LDCVs in chromaffin cells but not SVs in hippocampal neurons. How this would be achieved at the molecular level is unclear. Nevertheless, subtle differences exist between the priming machineries of chromaffin cells and neurons. For instance, chromaffin cells and neurons differ in their requirement for the Munc18-1/closed syntaxin complex on docking. In mice expressing open syntaxin-1B in the syntaxin-1A KO background, a docking defect was observed in chromaffin cells but not in cortical neurons (Gerber et al., 2008). It was thus postulated that in neurons, other active zone proteins absent in chromaffin cells mediate docking. It is tempting to speculate that the differential equipment of different cell types with 
Munc13 and CAPS isoforms may shape the $\mathrm{Ca}^{2+}$-dependence of priming. More work is required to decipher the nature of this residual priming in Munc13-deficient chromaffin cells and to shed light on the complex interdependence of Munc13s and CAPS protein function.

\subsection{Possible Mechanisms of Munc13 Function}

\subsubsection{Facilitation of SNARE Complex Formation and Tethering of Vesicular Membranes}

What is the molecular function of Munc13 in priming vesicles into releasable pools? Neuronal Munc13s have been reported to facilitate the formation of the SNARE complex, and various mechanistic models have been suggested. Munc13-4, a non-neuronal isoform, accelerates liposome fusion starting with SNAP-25/syntaxin-1 heterodimeric acceptor complexes (Boswell et al., 2012). The MUN domain of Munc13s appears to bind both membrane-anchored SNARE complexes and membrane-anchored syntaxin-1/SNAP-25 heterodimers (Guan et al., 2008), and has been shown to accelerate SNARE complex formation, starting from Munc18/closed syntaxin-1 complexes (Ma et al., 2011). In this case, Munc13 was suggested to extract the C-terminal SNARE motif of syntaxin-1 from the closed conformation, thus "opening" syntaxin-1 to allow for SNARE complex formation. Munc13s may therefore increase pool sizes by facilitating the formation and stabilisation of t-SNARE acceptor complexes on the plasma membrane (an early step in exocytosis) as well as the maintenance of primed vesicles on the plasma membrane. Another mechanism not mutually exclusive with a role in SNARE complex formation is that Munc13s may tether vesicles in the vicinity of release sites. A region around the MHD1 and MHD2 is structurally very similar to CATCHR family members ( $\mathrm{Li}$ et al., 2011). CATCHRs are proteins with a membrane tethering role which work in concert with SNARE proteins in diverse cellular membrane trafficking reactions (James and Martin, 2013). It would be interesting to see if the number of docked vesicles increases when Munc13 is overexpressed, and if increased SNARE complex formation in vivo underlies the increase in pool sizes that occurs upon Munc13 overexpression. However, the number of SNARE complexes formed per vesicle is expected to control the speed of release (Mohrmann et al., 2010; Mohrmann and Sorensen, 2012). As the speed of release was not increased upon Munc13 overexpression in chromaffin cells, this argues against an increase in the number of SNARE complexes formed per vesicle, 
i.e. there may be another factor that limits the number of SNARE complex that form in the presence of excess Munc13.

\subsubsection{Munc13 as a Target of PIP}

In neurosecretory cells, both syntaxin-1 and SNAP-25 are arranged in supramolecular clusters on the plasma membrane (Mohrmann and Sorensen, 2012). Syntaxin-1 clusters are maintained by weak homophilic interactions and ionic protein-lipid interactions (Sieber et al., 2007; van den Bogaart et al., 2011). Both cholesterol and $\mathrm{PIP}_{2}$ have been implicated in syntaxin-1 cluster formation, but the clusters that are formed by cholesterol are smaller than those by PIP $_{2}$ (van den Bogaart et al., 2011). The degree of overlap between SNAP-25 and syntaxin-1 clusters varies between reports (Lang et al., 2001; Nagy et al., 2005; Rickman et al., 2010), but no evidence has been provided that cluster formation hinders heterodimer formation (Rickman et al., 2010). In live PC12 cells, t-SNARE heterodimers were reported to exist in two configurations, one with a three-helix bundle involving both SNARE motifs of SNAP-25 and the other with only one SNAP-25 SNARE motif (Rickman et al., 2010). Complexes with these two configurations are spatially segregated from each other on the membrane. Since PIP 2 clusters and syntaxin-1 clusters colocalise to release sites (van den Bogaart et al., 2011), Munc13 may be recruited to these sites by $\mathrm{PIP}_{2}-\mathrm{C}_{2} \mathrm{~B}$ domain interactions in a $\mathrm{Ca}^{2+}$-dependent manner and facilitate and/or stabilise t-SNARE complex configurations that are more favourable for SNARE complex formation. In chromaffin cells, the size of the exocytotic burst scales with intracellular $\left[\mathrm{Ca}^{2+}\right]$ in the nanomolar range. Lawns of plasma membrane $\mathrm{PIP}_{2}$ have been reported to control pool sizes but not the time constants of release in chromaffin cells (Milosevic et al., 2005). Increasing plasma $\mathrm{PIP}_{2}$ concentrations by overexpression of phosphatidylinositol-4-phosphate-5-kinase $\mathrm{I} \gamma$ or infusion of $\mathrm{PIP}_{2}$ potentiates release from all secretion components, and conversely expression of an inositol 5phosphatase domain of synaptojanin 1 completely abolishes secretion. The latter result implies that physiological or basal levels of plasma membrane $\mathrm{PIP}_{2}$ are important for secretion in chromaffin cells. Since abrogation of endogenous Munc13 causes pool sizespecific changes and Munc13 recruitment to the plasma membrane is regulated by $\mathrm{PIP}_{2}$ at increased $\left[\mathrm{Ca}^{2+}\right]$ (Kabachinski et al., 2013), Munc13 may be a $\mathrm{PIP}_{2}$ target, regulating pool

sizes via its $\mathrm{C}_{2} \mathrm{~B}$ domain. Since the lipid microenvironment is thought to pattern the distribution of t-SNARE complexes with one vs. two SNAP-25 SNARE motifs (Rickman et 
al., 2010), it would therefore be interesting to also examine the impact Munc13 levels may have on the two populations of t-SNARE heterodimers.

\subsection{Possible Functions of Baiap3}

The function of Baiap3 remains enigmatic. Despite its expression in the adrenal gland in perinatal and adult mice (Fig. 3.1D, H), different experimental approaches showed no evidence of its positive or negative role in LDCV exocytosis in chromaffin cells (Fig. 3.912). At present, we can only speculate on its possible functions in the adrenal medulla and in the brain.

Munc13s are characterised by the presence of a MUN domain, which shows weak but significant homology to vesicle tethering complexes (Pei et al., 2009). Part of the MUN domain was shown to be structurally similar to vesicle tethering factors (Li et al., 2011). Hence, Munc13s and tethering factors were suggested to have a common role in membrane trafficking events. In phylogenetic trees demonstrating sequence homologies among the MUN domains of Munc13s and vesicle tethering factors, Munc13-1, Munc13-2 and Munc133 cluster in the same group, which is distinct from the group where Munc13-4 clusters together with Baiap3 (Pei et al., 2009). AEX-1 is a protein in C. elegans that was clustered in the same group with Baiap3 and Munc13-4. AEX-1 was documented to locate to muscles where it retrogradely stimulates pre-synaptic acetylcholine transmission across the neuromuscular junction by altering the localisation of pre-synaptic unc-13 (Doi and Iwasaki, 2002). Since the prohormone convertase AEX-5 is required for AEX-1 activity, the authors proposed that AEX-1 was involved in vesicular exocytosis of a peptidergic signal from muscles that modulates pre-synaptic exocytosis. Interestingly, Baiap3 was initially identified in a yeast two-hybrid screen as an interacting partner of the brain-specific angiogenesis inhibitor-1 (Shiratsuchi et al., 1998), a seven-span transmembrane receptor which has recently been shown to be enriched in the post-synaptic density (Stephenson et al., 2013). However, in the brain Baiap3 does not co-localize with the post-synaptic markers of glutamatergic or GABAergic synapses, and shows only limited co-localization with presynaptic glutamatergic and GABAergic markers (Wojcik et al., 2013). 


\subsubsection{Possible Functions of Baiap3 in the Adrenal Medulla}

Since Baiap3 does not appear to be present at most glutamatergic or GABAergic synapses, it may instead be involved in the release of peptidergic signals to modulate neurosecretory behaviour. In chromaffin cells, catecholamine release displays stress-induced plasticity, which was inhibited by neuropeptide Y (NPY) in an autocrine manner and possibly by other neurons localised in the adrenal medulla, such as NPY-synthesising type I ganglion neurons (Holgert et al., 1996; Wang et al., 2013). This plasticity is dependent upon an increase in tyrosine hydroxylase (TH) expression to support catecholamine synthesis. Interestingly, an increase in TH expression was observed in NPY KO mice, indicating that tonic release of NPY exists to control TH levels in chromaffin cells under basal conditions (Wang et al., 2013). It has been shown that in chromaffin cells, two populations of LDCVs differing in size and the "looseness" of vesicle membranes around the dense core exist (Grabner et al., 2005). In addition, although near-simultaneous quantal release of catecholamines and NPY has been observed, they represent only a fraction of all events and during many events either catecholamines or NPY were released (Whim, 2006). Together with a limited overlap of NPY and dopamine $\beta$ hydroxylase (the enzyme which catalyses the formation of norepinephrine from dopamine) immunoreactivity, this indicates that NPY and catecholamines can exist in different LDCVs in chromaffin cells (Whim, 2006). In addition, background amperometric signals in chromaffin cells were "vanishingly low" (Zhou and Misler, 1995), showing that if there is tonic vesicle exocytosis under basal conditions, these vesicles do not contain catecholamines. Therefore, Baiap3 may control the regulated or tonic release of neuropeptide-containing vesicles from chromaffin cells, which may exhibit release properties different from those of catecholamine-containing LDCVs. These vesicles may not exist as readily releasable pools, as catecholamine-containing LDCVs do, and their relative abundance to catecholamine-containing vesicles has yet to be established. Alternatively, Baiap3 may control the release of neuropeptides from type I ganglion neurons in the adrenal medulla to modulate catecholamine release from chromaffin cells.

\subsubsection{Possible Functions of Baiap3 in the Hypothalamus and Amygdala}

Baiap3 KO mice showed an increased propensity to pentylenetetrazole (PTZ)-induced seizures and an increased novelty-induced anxiety, which mostly manifested as an increased freezing response when placed in a novel (fear-conditioning) chamber (Wojcik et al., 2013). 
In addition, the action potential frequency recorded in the ventromedial nucleus of hypothalamic slices of Baiap3 KO mice was increased compared to that of WT mice, indicating an increase in basal network activity which is consistent with the increased seizure propensitiy. Together with an altered response to benzodiazepines, this suggests an altered balance of excitation and inhibition in Baiap3 $\mathrm{KO}$ mice. The ventromedial nucleus of the hypothalamus is innervated by the anterior, postereodorsal and postereoventral medial amydaloid nucleus (Pardo-Bellver et al., 2012). It also receives afferent input from the paraventricular nucleus of the hypothalamus and the adjacent posterior part of the bed nucleus of stria terminalis (Chen et al., 2011). Most of the above structures also express Baiap3 (Wojcik et al., 2013). Since the amygdala and the hypothalamus are implicated in the regulation of anxiety and fear responses (Sajdyk et al., 2004), and Baiap3 is expressed abundantly in both structures, it may function in regulating the release of neuropeptides in these structures. Given that a large number of neuropeptides are involved in mediating anxiety and fear responses in the amygdala and hypothalamus, for instance NPY, corticotropin-releasing factor, vasopressin, oxytocin, melanin concentrating hormone and galanin, to name a few (Alldredge, 2010), there are many possible candidates for Baiap3mediated regulation. Baiap3 may be involved in priming, similar to other Munc13 isoforms, but since it is only distantly related to the other neuronal isoforms (Munc13-1, Munc13-2 and Munc13-3) and possesses the CATCHR domain present in vesicle tethering factors (James and Martin, 2013), it may also be involved in mediating other cellular membrane trafficking events.

\subsection{The Choice of Munc13 Isoforms as Priming Factors in Chromaffin Cells and Synapses}

Our data show that ubMunc13-2 is the dominant Munc13 isoform in the control of LDCV pool sizes in chromaffin cells, with Munc13-1 playing a minor role (Fig. 3.5). This is obviously distinct from the case of hippocampal glutamatergic neurons, where neurotransmitter release from SVs at $90 \%$ of synapses depends on Munc13-1 and the remainder on Munc13-2 (Rosenmund et al., 2002). What determines the equipment of neurosecretory cells with different Munc13 isoforms? Many inherent differences exist in the release properties between these two cell types. In hippocampal neurons, a single action potential would cause robust synchronous neurotransmitter secretion from SVs in less than 1 
ms. In contrast, in chromaffin cells, catecholamine release detected as amperometric spikes occurred with delays that are variable between cells (after 2 or 15 action potentials), and widely spaced action potentials are relatively ineffective in evoking release (Zhou and Misler, 1995). Furthermore, in chromaffin cells, the maximum rate of release is ten-fold lower than that of a glutamatergic terminal such as the calyx of Held synapse (Neher, 2006). Thus, there are significant physiological differences in the excitation-secretion coupling between neurons and chromaffin cells. In neurons, exocytosis of SVs is largely limited to specialised active zones, whereas DCVs can be released from both synaptic and extrasynaptic sites (van de Bospoort et al., 2012). In hippocampal neurons, synaptic DCV release is dependent on Munc13s and overexpression of Munc13-1 in synapses shifts the synaptic preference of DCV release to extrasynaptic sites (van de Bospoort et al., 2012). The primarily synaptic localization of endogenous Munc13-1 and Munc13-2 in neurons thus appears to promote the synaptic release of both SVs and DCVs. However, in chromaffin cells LDCV exocytosis can occur across the entire cell surface. Chromaffin cells therefore contain more release sites than a single synapse allowing pool sizes to be highly modulated (Neher, 2006). Last but not least, in addition to depolarisation-induced release through the activation of nicotinic acetylcholine (ACh) receptor channels in chromaffin cells, in vivo acetylcholine activates muscarinic ACh receptors, which act through PLC activation to release $\mathrm{Ca}^{2+}$ from intracellular $\mathrm{Ca}^{2+}$ stores, thus contributing to asynchronous release (Misler, 2009). The distinct release properties of chromaffin cells and neurons, although both cell types largely employ the same set of proteins, imply that they have different requirements with respect to the vesicle pool refilling machinery. As shown in Fig. 3.8, in chromaffin cells ubMunc13-2 is more efficient in priming than Munc13-1. This is in agreement with previous findings that pool recovery is faster in ubMunc13-2-overexpressing WT chromaffin cells than Munc13-1-overexpressing WT cells (Zikich et al., 2008), and with the more prominent short-term enhancement mediated by ubMunc13-2 in autaptic hippocampal synapses (Rosenmund et al., 2002). The equipment of chromaffin cells with ubMunc13-2 as the dominant priming factor probably satisfies the physiological requirements of catecholamine release. While in neurons, highly synchronised excitation-secretion coupling is critical for the encoding of information, in chromaffin cells a sustained response that allows the release of large amounts of catecholamines into the blood when needed is probably more important. The coupling of release to action potentials exists in chromaffin cells as well (Zhou and Misler, 1995), but is not as tight as in neurons, and release of chatecholamines occurs in bulk into the bloodstream. The equipment of chromaffin cells with ubMunc13-2 as the dominant factor in regulating 
LDCV exocytosis probably ensures that the supply of releasable vesicles is high for increased demands. In synapses, instead of providing sufficient vesicles for bulk release, a balance between the provision of sufficient vesicles at high synaptic activity and synchronicity of release is more important to neurons as components of a computing network.

The mild increase of the SRP observed in Munc13-1 KO chromaffin cells may reflect the competition between Munc13-1 and ubMunc13-2 in priming LDCVs in chromaffin cells (Fig. 3.2). If such a competition exists in neurons, it may be a mechanism by which different Munc13 isoforms determine synaptic plasticity characteristics in a given type of neuron. In this context, it would be also interesting to examine possible dynamic changes in the expression levels of Munc13 isoforms in the brain and in the adrenal gland in vivo under pathological conditions or in response to experimental manipulation. However, different synapses/neurons have been shown to display functional specialisation in their "usage" of Munc13 isoforms. For example, Munc13-2 mediates facilitation in autaptic hippocampal cultures (Rosenmund et al., 2002) (assumed to contain predominantly pyramidal cells by Breustedt et al., 2010) whereas hippocampal mossy fiber synapses selectively show facilitation in the absence of Munc13-2 (Breustedt et al., 2010; Rosenmund et al., 2002). In the analysis of the summation of Munc13 effects, it is therefore important to consider the expression and subcellular localization of all Munc13 isoforms in a given cell. Another point of interest is whether cognate SNARE proteins exist for different Munc13 isoforms, which would add another layer of complexity to the system. TIRF microscopy studies have demonstrated that granules docked before stimulation, but not newcomer granules, fuse at syntaxin-1A clusters (Ohara-Imaizumi et al., 2007). It remains to be demonstrated whether Munc13-1 or other Munc13 isoforms preferentially interact with syntaxins other than syntaxin-1A. In summary, much additional work is required in trying to find a unifying theory on how Munc13 isoforms mediate vesicle priming in general and how they shape different forms of synaptic plasticity.

\subsection{Significance of Our Findings}

It had been suggested that CAPS/unc-31 is essential for LDCV exocytosis and Munc13s mainly mediates priming of SVs (Augustin et al., 1999b; Liu et al., 2008; Sieburth et al., 2007; Speese et al., 2007; Varoqueaux et al., 2002; Zhou et al., 2007). More recently, van de Bospoort et al. reported that in hippocampal neurons Munc13s regulate the 
preferential release of DCVs from synaptic rather than extrasynaptic sites (van de Bospoort et al., 2012). The current study establishes ubMunc13-2 as the main LDCV priming factor in mouse chromaffin cells and further demonstrates a contribution of endogenous Munc13-1 to LDCV exocytosis in this cell type. The absence of ubMunc13-2 has prominent effects on all components of release, and is more deleterious than the removal of both isoforms of CAPS. A comparison of the effects of Munc13 and CAPS deletion between SV release from hippocampal neurons and LDCV release from chromaffin cells shows that the phenotypes are in fact relatively similar, with Munc13 being the dominant priming factor and CAPS being dispensable under conditions of high $\left[\mathrm{Ca}^{2+}\right]$. In CAPS DKO chromaffin cells, the overfilling of the SRP triggered by a second flash stimulus is reminiscent of the transient reversal of release seen in CAPS DKO hippocampal neurons by increased $\left[\mathrm{Ca}^{2+}\right]$ (Liu et al., 2008). Munc13/CAPS most likely cooperate in both cell types in vesicle pool refilling in a similar fashion. Thus, the dichotomy between CAPS priming for LDCVs and Munc13 priming for SVs, at least in vertebrates, should not be considered valid and the two types of vesicles may have more similar exocytotic machineries than had been thought. In contrast to the large release deficits observed in Munc13-2-deficient animals in this study, at least two studies in C. elegans reported unperturbed DCV release (of insulin-like cargo and atrial natriuretic factor, a neuropeptide) in unc-13 null mutants, implying that in distantly related organisms, the employment of priming factors could be somewhat different. Another striking difference between C. elegans and mouse, is that unc-31 mutants show a DCV docking deficit that can be rescued by open syntaxin, while in CAPS DKO chromaffin cells a docking defect was absent and expression of open syntaxin in these cells in turn causes a docking defect (Hammarlund et al., 2008; Liu et al., 2010). In addition to the differences that appear to exist between species, DCVs represent a diverse group of intracellular organelles with a plethora of different cargos, which are released in response to the presence or absence of diverse physiological stimuli. Therefore, investigation of individual DCV systems is probably required before generalisations can be made with respect to the relative importance of similarly functioning components in DCV release. Theoretically, cells that rely mainly on priming by Munc13 to regulate pool sizes could be more readily subjected to regulation by $\mathrm{Ca}^{2+} /$ calmodulin, phorbol esters and plasmalemmal RIM concentrations compared to cells mainly reliant on CAPS for priming (e.g. DCVs from C. elegans neurons). With both Munc13 and CAPS being involved in priming of both types of vesicles, it would be interesting to examine how titrations of these two proteins, whose isoforms are found to show 
differential expression in the brain and various secretory systems, exert an effect on the $\mathrm{Ca}^{2+}$ dependence of priming and pool recovery in different systems. 


\section{Supplementary Materials}

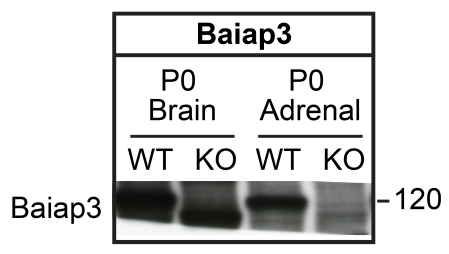

Supplementary Fig. 1. Overnight exposure of the blot showing possible expression of the truncated product of Baiap3 at low levels.

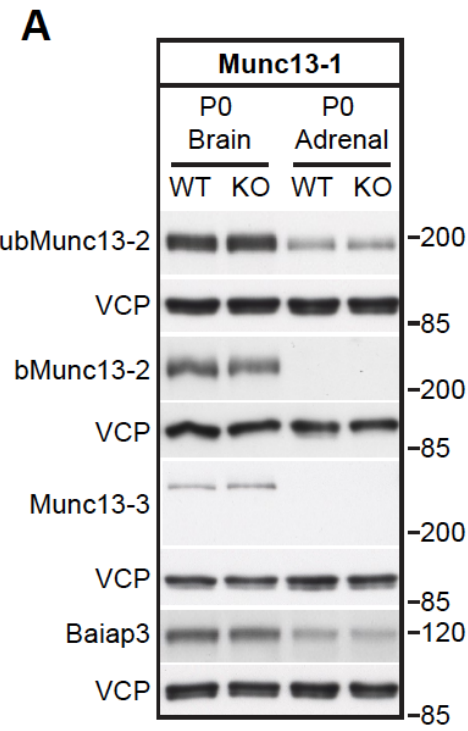

B

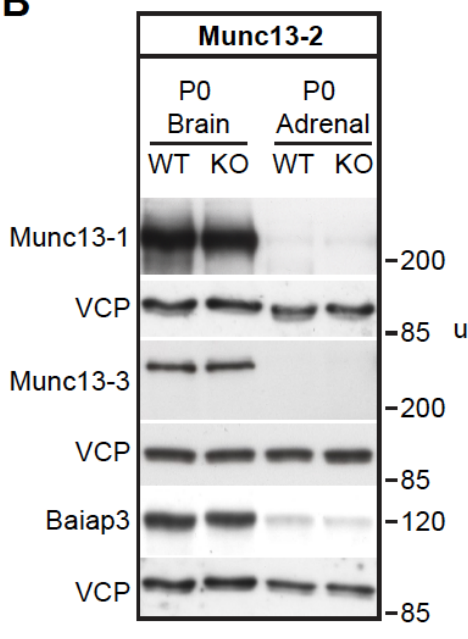

C

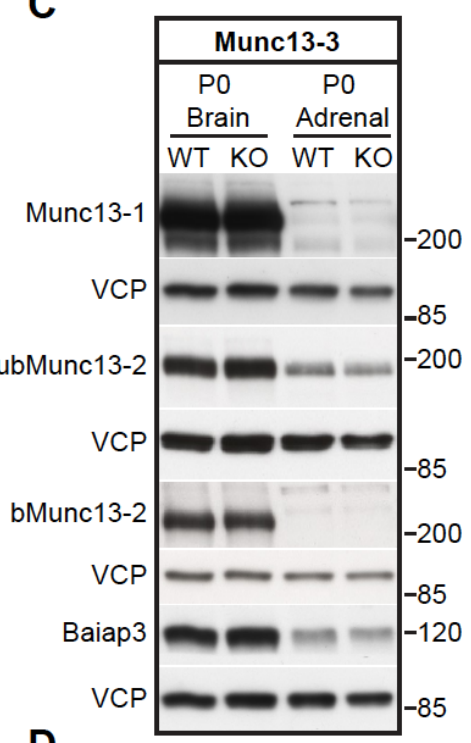

D

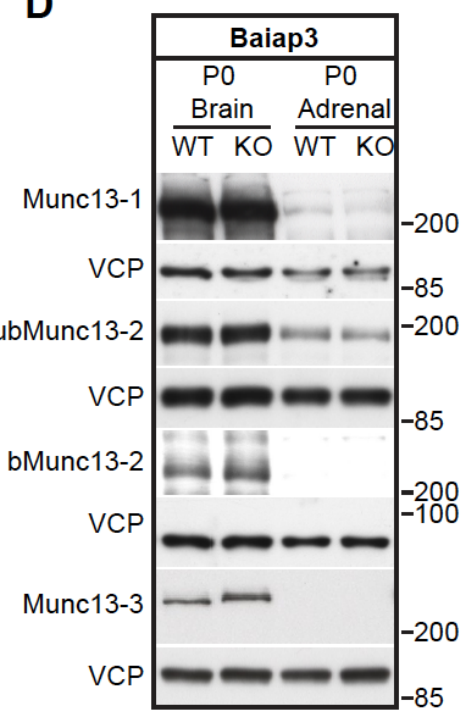

Supplementary Fig. 2. Expression of Munc13 isoforms in brain and adrenal glands of (A) E18 Munc13-1 KO mice, (B) P0 Munc13-2 KO mice, (C) P0 Munc13-3 KO mice, and (D) P0 Baiap3 KO mice. 


\begin{tabular}{|c|c|c|c|c|c|}
\hline Pre-flash & Control & $\begin{array}{l}\text { Munc13-1 } 1^{-/-} \\
\text {Munc13-2 }\end{array}$ & $\begin{array}{l}\text { Munc13-1 } \\
\text { Munc13- } 13-2^{-/-}\end{array}$ & $\begin{array}{l}\text { Munc13-1 } \\
\text { Munc1- } 13-2^{-/-}\end{array}$ & $\begin{array}{l}\text { Munc13-1 } 1^{-/-} \\
\text {Munc13-2 }\end{array}$ \\
\hline Control & & & & & \\
\hline Munc13-1 $1^{-/-}$Munc13-2 & & & & & \\
\hline Munc13-1 ${ }^{+/+}$Munc13-2 $2^{-/-}$ & & & & & \\
\hline Munc13-1 $1^{+/-}$Munc13-2 & & & & & \\
\hline Munc13-1 $1^{-/-}$Munc13-2-/- & & & & & \\
\hline
\end{tabular}

\begin{tabular}{|c|c|c|c|c|c|}
\hline Post-flash 1 & Control & $\begin{array}{l}\text { Munc13-1 } 1^{-/-} \\
\text {Munc13-2 }\end{array}$ & $\begin{array}{l}\text { Munc13-1 } \\
\text { Munc13-1 } \\
\text { M-t- }\end{array}$ & $\begin{array}{l}\text { Munc13-1 } \\
\text { Munc1-- } 13-2^{-/-}\end{array}$ & $\begin{array}{l}\text { Munc13-1 } 1^{-/-} \\
\text {Munc13-2 }\end{array}$ \\
\hline Control & & & & 0.00165 & \\
\hline Munc13-1/- Munc13-2 $2^{+/-}$ & & & & 0.00964 & \\
\hline Munc13-1 $1^{+/+}$Munc13-2 ${ }^{-/-}$ & & & & 0.04452 & \\
\hline Munc13-1 $1^{+/-}$Munc13-2/- & & & & & \\
\hline Munc13-1/-Munc13-2/- & & & & & \\
\hline
\end{tabular}

\begin{tabular}{|c|c|c|c|c|c|}
\hline Post-flash 2 & Control & $\begin{array}{l}\text { Munc13-1-1- } \\
\text { Munc13-2 }\end{array}$ & $\begin{array}{l}\text { Munc13-1 } 1^{+/+} \\
\text {Munc13-2 }\end{array}$ & $\begin{array}{l}\text { Munc13-1 } 1^{+/-} \\
\text {Munc13-2 }\end{array}$ & $\begin{array}{l}\text { Munc13-1-1- } \\
\text { Munc13-2- }\end{array}$ \\
\hline Control & & & & 0.00799 & \\
\hline Munc13-1 $1^{-/}$Munc13-2 $2^{+/-}$ & & & & 0.03777 & \\
\hline Munc13-1 $1^{+/+}$Munc13-2 & & & & 0.00282 & \\
\hline Munc13-1 ${ }^{+/-}$Munc13-2 $2^{-/-}$ & & & & & 0.00423 \\
\hline Munc13-1 $1^{-/-}$Munc 13-2 $2^{-/-}$ & & & & & \\
\hline
\end{tabular}

\begin{tabular}{|c|c|c|c|c|c|}
\hline Post-flash 3 & Control & $\begin{array}{l}\text { Munc13-1-1- } \\
\text { Munc13-2 }\end{array}$ & $\begin{array}{l}\text { Munc13-1 } \\
\text { Munc13- } 13-2^{-/-}\end{array}$ & $\begin{array}{l}\text { Munc13-1 } \\
\text { Munc13-- }\end{array}$ & $\begin{array}{l}\text { Munc13-1-1- } \\
\text { Munc13-2 }\end{array}$ \\
\hline Control & & 0.02558 & & 0.00551 & \\
\hline Munc13-1 $1^{-/-}$Munc13-2 $2^{+/-}$ & & & 0.02968 & & 0.00577 \\
\hline Munc13-1 $1^{+/+}$Munc13-2/- & & & & 0.00688 & \\
\hline Munc13-1 $1^{+/-}$Munc13-2/- & & & & & 0.000158 \\
\hline Munc13-1 $1^{-/-}$Munc13-2 $2^{-/-}$ & & & & & \\
\hline
\end{tabular}

\begin{tabular}{|c|c|c|c|c|c|}
\hline Post-flash 4 & Control & $\begin{array}{l}\text { Munc13-1 } 1^{-/-} \\
\text {Munc13-2 }\end{array}$ & $\begin{array}{l}\text { Munc13-1 } \\
\text { Munc13-2 } \\
\text { +/- }\end{array}$ & $\begin{array}{l}\text { Munc13-1 } \\
\text { Munc13-- } \\
\text { Mu- }\end{array}$ & $\begin{array}{l}\text { Munc13-1-1- } \\
\text { Munc13-2 }\end{array}$ \\
\hline Control & & 0.02522 & & 0.00193 & \\
\hline Munc13-1 $1^{-/-}$Munc13-2 ${ }^{+/-}$ & & & 0.00502 & & 0.000417 \\
\hline Munc13-1 $1^{+/+}$Munc13-2/- & & & & 0.000318 & \\
\hline Munc13-1 $1^{+/-}$Munc13-2/- & & & & & $<0.0001$ \\
\hline Munc13-1 $1^{-/-}$Munc13-2 $2^{-/-}$ & & & & & \\
\hline
\end{tabular}

\begin{tabular}{|c|c|c|c|c|c|}
\hline Post-flash 5 & Control & $\begin{array}{l}\text { Munc13-1-1- } \\
\text { Munc13-2 } 2^{+-}\end{array}$ & $\begin{array}{l}\text { Munc13-1 } 1^{+/+} \\
\text {Munc13-2 }\end{array}$ & $\begin{array}{l}\text { Munc13-1 } 1^{+/-} \\
\text {Munc13-2 }\end{array}$ & $\begin{array}{l}\text { Munc13-1-1- } \\
\text { Munc13-2- }\end{array}$ \\
\hline Control & & 0.01667 & & 0.00290 & 0.01883 \\
\hline Munc13-1 $1^{-/}$Munc13-2 & & & 0.00162 & & $<0.0001$ \\
\hline Munc13-1 ${ }^{+/+}$Munc13-2/- & & & & 0.00027 & \\
\hline Munc13-1 $1^{+/-}$Munc13-2 $2^{-/-}$ & & & & & $<0.0001$ \\
\hline Munc13-1 ${ }^{-/-}$Munc13-2 $2^{-/-}$ & & & & & \\
\hline
\end{tabular}




\begin{tabular}{|c|c|c|c|c|c|}
\hline Post-flash 6 & Control & $\begin{array}{l}\text { Munc13-1 } 1^{-/-} \\
\text {Munc13-2 }\end{array}$ & $\begin{array}{l}\text { Munc13-1 } \\
\text { Munc13- } 13-2^{-/-}\end{array}$ & $\begin{array}{l}\text { Munc13-1 } 1^{+/-} \\
\text {Munc13-2 }\end{array}$ & $\begin{array}{l}\text { Munc13-1-1- } \\
\text { Munc13-2-/ }\end{array}$ \\
\hline Control & & 0.03230 & & 0.00907 & 0.01008 \\
\hline Munc13-1 $1^{-/-}$Munc13-2 & & & 0.00202 & & $<0.0001$ \\
\hline Munc13-1 ${ }^{+/+}$Munc13-2/- & & & & 0.00058 & \\
\hline Munc13-1 $1^{+/-}$Munc13-2 $2^{-/-}$ & & & & & $<0.0001$ \\
\hline Munc13-1 ${ }^{-/-}$Munc $13-2^{-/-}$ & & & & & \\
\hline
\end{tabular}

\begin{tabular}{|c|c|c|c|c|c|}
\hline Post-flash 7 & Control & $\begin{array}{l}\text { Munc13-1-1- } \\
\text { Munc13-2 }\end{array}$ & $\begin{array}{l}\text { Munc13-1 } 1^{+/+} \\
\text {Munc13-2 }\end{array}$ & $\begin{array}{l}\text { Munc13-1 } \\
\text { Munc13-- } 2^{-/-}\end{array}$ & $\begin{array}{l}\text { Munc13-1-1- } \\
\text { Munc13-2/ }\end{array}$ \\
\hline Control & & 0.03509 & & 0.00814 & 0.00952 \\
\hline Munc13-1 $1^{-/-}$Munc13-2 ${ }^{+/-}$ & & & 0.00237 & & $<0.0001$ \\
\hline Munc13-1 ${ }^{+/+}$Munc13-2/- & & & & 0.000421 & \\
\hline Munc13-1 $1^{+/-}$Munc13-2 & & & & & $<0.0001$ \\
\hline Munc13-1 $1^{-/-}$Munc13-2 & & & & & \\
\hline
\end{tabular}

\begin{tabular}{|c|c|c|c|c|c|}
\hline Post-flash 8 & Control & $\begin{array}{l}\text { Munc13-1 } 1^{-/-} \\
\text {Munc13-2 }\end{array}$ & $\begin{array}{l}\text { Munc13-1 } \\
\text { Munc13-2-1- }\end{array}$ & $\begin{array}{l}\text { Munc13-1 } \\
\text { Munc13-- }\end{array}$ & $\begin{array}{l}\text { Munc13-1 } \\
\text { Munc13-2- }\end{array}$ \\
\hline Control & & & & 0.01443 & 0.00898 \\
\hline Munc13-1 $1^{-/-}$Munc13-2 ${ }^{+/-}$ & & & 0.00306 & & 0.00016 \\
\hline Munc13-1 $1^{+/+}$Munc13-2/- & & & & 0.00040 & \\
\hline Munc13-1 $1^{+/-}$Munc13-2/- & & & & & $<0.0001$ \\
\hline Munc13-1/-Munc13-2 $2^{-/-}$ & & & & & \\
\hline
\end{tabular}

Supplementary Table 1. $P$-values obtained for differences in pre-flash and post-flash $\left[\mathrm{Ca}^{2+}\right]$ among experimental groups during capacitance measurements to indicate the amount of LDCV exocytosis in Munc13-1 $1^{-/}$Munc13-2 ${ }^{+/-}$, Munc13-1 ${ }^{+/+}$Munc13-2 $2^{-/-}$, Munc13-1 ${ }^{+/-}$ Munc13-2-/- and Munc13-1/2 DKO cells using Munc13-1 ${ }^{+/+} /$Munc13-1 $1^{+/-}$Munc13-2 ${ }^{+/-}$cells as control. Values in pink boxes indicate that the group in the left-most column showed a higher $\left[\mathrm{Ca}^{2+}\right]$ than the group in the top row for a given comparison. Values in light green boxes indicate that the group in the top row showed a higher $\left[\mathrm{Ca}^{2+}\right]$ than the group in the left-most column.

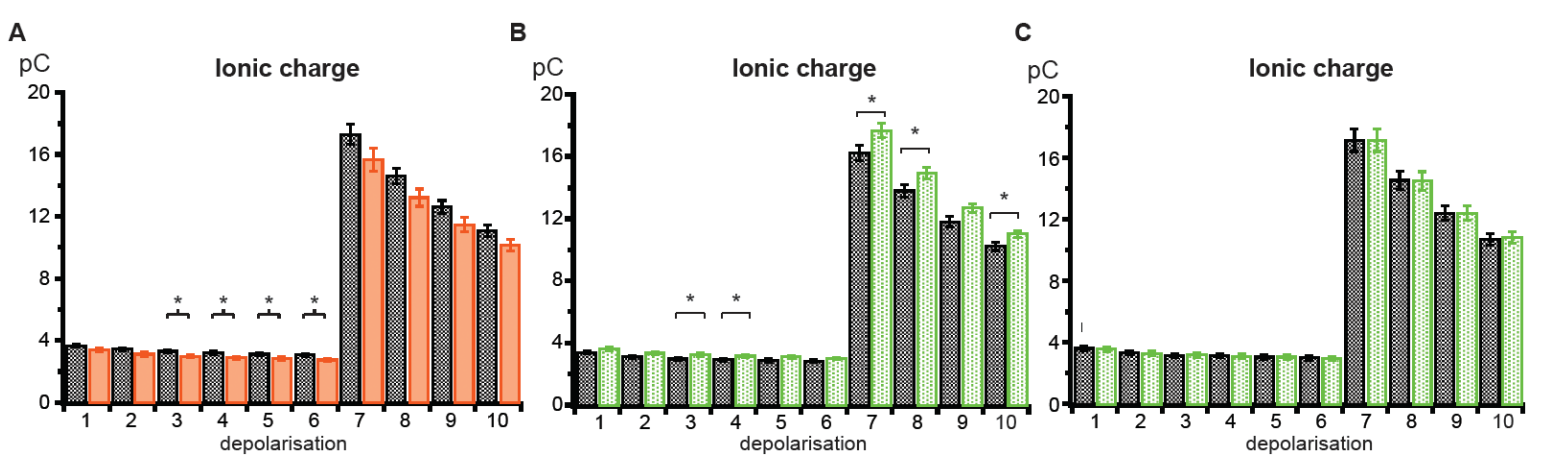

Supplementary Fig. 3. Ionic charge in each depolarisation for (A) Munc13-2 WT and KO cells, (B) Baiap3 WT and KO cells before matching for similar amounts of ionic charge and (C) Baiap3 WT and KO cells after matching for similar amounts of ionic charge during the first $100 \mathrm{~ms}$ depolarisation. 


\section{References}

Abbott, L.F., and W.G. Regehr. 2004. Synaptic computation. Nature. 431:796-803.

Ahras, M., G.P. Otto, and S.A. Tooze. 2006. Synaptotagmin IV is necessary for the maturation of secretory granules in PC12 cells. J Cell Biol. 173:241-251.

Alabi, A.A., and R.W. Tsien. 2012. Synaptic vesicle pools and dynamics. Cold Spring Harb Perspect Biol. 4:a013680.

Alldredge, B. 2010. Pathogenic involvement of neuropeptides in anxiety and depression. Neuropeptides. 44:215-224.

Aravamudan, B., T. Fergestad, W.S. Davis, C.K. Rodesch, and K. Broadie. 1999. Drosophila UNC-13 is essential for synaptic transmission. Nat Neurosci. 2:965-971.

Ashery, U., A. Betz, T. Xu, N. Brose, and J. Rettig. 1999. An efficient method for infection of adrenal chromaffin cells using the Semliki Forest virus gene expression system. Eur J Cell Biol. 78:525-532.

Ashery, U., F. Varoqueaux, T. Voets, A. Betz, P. Thakur, H. Koch, E. Neher, N. Brose, and J. Rettig. 2000. Munc13-1 acts as a priming factor for large dense-core vesicles in bovine chromaffin cells. Embo j. 19:3586-3596.

Augustin, I., A. Betz, C. Herrmann, T. Jo, and N. Brose. 1999a. Differential expression of two novel Munc13 proteins in rat brain. Biochem J. 337 ( Pt 3):363-371.

Augustin, I., S. Korte, M. Rickmann, H.A. Kretzschmar, T.C. Sudhof, J.W. Herms, and N. Brose. 2001. The cerebellum-specific Munc13 isoform Munc13-3 regulates cerebellar synaptic transmission and motor learning in mice. J Neurosci. 21:10-17.

Augustin, I., C. Rosenmund, T.C. Sudhof, and N. Brose. 1999b. Munc13-1 is essential for fusion competence of glutamatergic synaptic vesicles. Nature. 400:457-461.

Banerjee, A., V.A. Barry, B.R. DasGupta, and T.F. Martin. 1996. N-Ethylmaleimidesensitive factor acts at a prefusion ATP-dependent step in Ca2+-activated exocytosis. $J$ Biol Chem. 271:20223-20226.

Basu, J., A. Betz, N. Brose, and C. Rosenmund. 2007. Munc13-1 C1 domain activation lowers the energy barrier for synaptic vesicle fusion. J Neurosci. 27:1200-1210.

Basu, J., N. Shen, I. Dulubova, J. Lu, R. Guan, O. Guryev, N.V. Grishin, C. Rosenmund, and J. Rizo. 2005. A minimal domain responsible for Munc13 activity. Nat Struct Mol Biol. 12:1017-1018.

Betz, A., U. Ashery, M. Rickmann, I. Augustin, E. Neher, T.C. Sudhof, J. Rettig, and N. Brose. 1998. Munc13-1 is a presynaptic phorbol ester receptor that enhances neurotransmitter release. Neuron. 21:123-136.

Betz, A., M. Okamoto, F. Benseler, and N. Brose. 1997. Direct interaction of the rat unc13 homologue Munc13-1 with the N terminus of syntaxin. J Biol Chem. 272:2520-2526. 
Betz, A., P. Thakur, H.J. Junge, U. Ashery, J.S. Rhee, V. Scheuss, C. Rosenmund, J. Rettig, and N. Brose. 2001. Functional interaction of the active zone proteins Munc13-1 and RIM1 in synaptic vesicle priming. Neuron. 30:183-196.

Betz, W.J., and A.W. Henkel. 1994. Okadaic acid disrupts clusters of synaptic vesicles in frog motor nerve terminals. J Cell Biol. 124:843-854.

Boswell, K.L., D.J. James, J.M. Esquibel, S. Bruinsma, R. Shirakawa, H. Horiuchi, and T.F. Martin. 2012. Munc13-4 reconstitutes calcium-dependent SNARE-mediated membrane fusion. J Cell Biol. 197:301-312.

Breustedt, J., A. Gundlfinger, F. Varoqueaux, K. Reim, N. Brose, and D. Schmitz. 2010. Munc13-2 differentially affects hippocampal synaptic transmission and plasticity. Cerebral cortex (New York, N.Y. : 1991). 20:1109-1120.

Bruns, D. 2004. Detection of transmitter release with carbon fiber electrodes. Methods. 33:312-321.

Burgalossi, A., S. Jung, G. Meyer, W.J. Jockusch, O. Jahn, H. Taschenberger, V.M. O'Connor, T. Nishiki, M. Takahashi, N. Brose, and J.S. Rhee. 2010. SNARE protein recycling by alphaSNAP and betaSNAP supports synaptic vesicle priming. Neuron. 68:473-487.

Burkhardt, P., D.A. Hattendorf, W.I. Weis, and D. Fasshauer. 2008. Munc18a controls SNARE assembly through its interaction with the syntaxin N-peptide. EMBO J. 27:923933.

Capogna, M., R.A. McKinney, V. O'Connor, B.H. Gahwiler, and S.M. Thompson. 1997. Ca2+ or Sr2+ partially rescues synaptic transmission in hippocampal cultures treated with botulinum toxin A and C, but not tetanus toxin. J Neurosci. 17:7190-7202.

Ceccarelli, B., W.P. Hurlbut, and A. Mauro. 1972. Depletion of vesicles from frog neuromuscular junctions by prolonged tetanic stimulation. J Cell Biol. 54:30-38.

Chen, P., D. Lin, J. Giesler, and C. Li. 2011. Identification of urocortin 3 afferent projection to the ventromedial nucleus of the hypothalamus in rat brain. The Journal of comparative neurology. 519:2023-2042.

Conner, S.D., and S.L. Schmid. 2003. Regulated portals of entry into the cell. Nature. 422:37-44.

Cooper, B., M. Hemmerlein, J. Ammermuller, C. Imig, K. Reim, N. Lipstein, S. Kalla, H. Kawabe, N. Brose, J.H. Brandstatter, and F. Varoqueaux. 2012. Munc13-independent vesicle priming at mouse photoreceptor ribbon synapses. J Neurosci. 32:8040-8052.

de Wit, H., L.N. Cornelisse, R.F. Toonen, and M. Verhage. 2006. Docking of secretory vesicles is syntaxin dependent. PLoS One. 1:e126.

de Wit, H., A.M. Walter, I. Milosevic, A. Gulyas-Kovacs, D. Riedel, J.B. Sorensen, and M. Verhage. 2009. Synaptotagmin-1 docks secretory vesicles to syntaxin-1/SNAP-25 acceptor complexes. Cell.138:935-946. 
Deng, L., P.S. Kaeser, W. Xu, and T.C. Sudhof. 2011. RIM proteins activate vesicle priming by reversing autoinhibitory homodimerization of Munc13. Neuron. 69:317-331.

Denker, A., and S.O. Rizzoli. 2010. Synaptic vesicle pools: an update. Front Synaptic Neurosci. 2:135.

Doi, M., and K. Iwasaki. 2002. Regulation of retrograde signaling at neuromuscular junctions by the novel C2 domain protein AEX-1. Neuron. 33:249-259.

Domanska, M.K., V. Kiessling, and L.K. Tamm. 2010. Docking and fast fusion of synaptobrevin vesicles depends on the lipid compositions of the vesicle and the acceptor SNARE complex-containing target membrane. Biophys J. 99:2936-2946.

Dulubova, I., M. Khvotchev, S. Liu, I. Huryeva, T.C. Sudhof, and J. Rizo. 2007. Munc18-1 binds directly to the neuronal SNARE complex. Proc Natl Acad Sci U S A. 104:2697-2702.

Dulubova, I., X. Lou, J. Lu, I. Huryeva, A. Alam, R. Schneggenburger, T.C. Sudhof, and J. Rizo. 2005. A Munc13/RIM/Rab3 tripartite complex: from priming to plasticity? Embo $j$. 24:2839-2850.

Dulubova, I., S. Sugita, S. Hill, M. Hosaka, I. Fernandez, T.C. Sudhof, and J. Rizo. 1999. A conformational switch in syntaxin during exocytosis: role of munc18. EMBO J. 18:43724382.

Dulubova, I., T. Yamaguchi, D. Arac, H. Li, I. Huryeva, S.W. Min, J. Rizo, and T.C. Sudhof. 2003. Convergence and divergence in the mechanism of SNARE binding by Sec1/Munc18-like proteins. Proc Natl Acad Sci U S A. 100:32-37.

Fasshauer, D., H. Otto, W.K. Eliason, R. Jahn, and A.T. Brunger. 1997. Structural changes are associated with soluble $\mathrm{N}$-ethylmaleimide-sensitive fusion protein attachment protein receptor complex formation. J Biol Chem. 272:28036-28041.

Fasshauer, D., R.B. Sutton, A.T. Brunger, and R. Jahn. 1998. Conserved structural features of the synaptic fusion complex: SNARE proteins reclassified as Q- and R-SNAREs. Proc Natl Acad Sci U S A. 95:15781-15786.

Feldmann, J., I. Callebaut, G. Raposo, S. Certain, D. Bacq, C. Dumont, N. Lambert, M. Ouachee-Chardin, G. Chedeville, H. Tamary, V. Minard-Colin, E. Vilmer, S. Blanche, F. Le Deist, A. Fischer, and G. de Saint Basile. 2003. Munc13-4 is essential for cytolytic granules fusion and is mutated in a form of familial hemophagocytic lymphohistiocytosis (FHL3). Cell. 115:461-473.

Fernandez-Alfonso, T., and T.A. Ryan. 2008. A heterogeneous "resting" pool of synaptic vesicles that is dynamically interchanged across boutons in mammalian CNS synapses. Brain Cell Biol. 36:87-100.

Fernandez, I., J. Ubach, I. Dulubova, X. Zhang, T.C. Sudhof, and J. Rizo. 1998. Threedimensional structure of an evolutionarily conserved N-terminal domain of syntaxin $1 \mathrm{~A}$. Cell. 94:841-849. 
Fornasiero, E.F., A. Raimondi, F.C. Guarnieri, M. Orlando, R. Fesce, F. Benfenati, and F. Valtorta. 2012. Synapsins contribute to the dynamic spatial organization of synaptic vesicles in an activity-dependent manner. J Neurosci. 32:12214-12227.

Fukuda, M. 2006. Rab27 and its effectors in secretory granule exocytosis: a novel docking machinery composed of a Rab27.effector complex. Biochem Soc Trans. 34:691695.

Geppert, M., Y. Goda, R.E. Hammer, C. Li, T.W. Rosahl, C.F. Stevens, and T.C. Sudhof. 1994. Synaptotagmin I: a major Ca2+ sensor for transmitter release at a central synapse. Cell. 79:717-727.

Gerber, S.H., J.C. Rah, S.W. Min, X. Liu, H. de Wit, I. Dulubova, A.C. Meyer, J. Rizo, M. Arancillo, R.E. Hammer, M. Verhage, C. Rosenmund, and T.C. Sudhof. 2008.

Conformational switch of syntaxin-1 controls synaptic vesicle fusion. Science. 321:15071510.

Grabner, C.P., S.D. Price, A. Lysakowski, A.L. Cahill, and A.P. Fox. 2006. Regulation of large dense-core vesicle volume and neurotransmitter content mediated by adaptor protein 3. Proc Natl Acad Sci U S A. 103:10035-10040.

Grabner, C.P., S.D. Price, A. Lysakowski, and A.P. Fox. 2005. Mouse chromaffin cells have two populations of dense core vesicles. J Neurophysiol. 94:2093-2104.

Graham, J.M. 2002. Preparation of crude subcellular fractions by differential centrifugation. ScientificWorldJournal. 2:1638-1642.

Guan, R., H. Dai, and J. Rizo. 2008. Binding of the Munc13-1 MUN domain to membraneanchored SNARE complexes. Biochemistry. 47:1474-1481.

Gulyas-Kovacs, A., H. de Wit, I. Milosevic, O. Kochubey, R. Toonen, J. Klingauf, M. Verhage, and J.B. Sorensen. 2007. Munc18-1: sequential interactions with the fusion machinery stimulate vesicle docking and priming. J Neurosci. 27:8676-8686.

Hammarlund, M., M.T. Palfreyman, S. Watanabe, S. Olsen, and E.M. Jorgensen. 2007. Open syntaxin docks synaptic vesicles. PLoS Biol. 5:e198.

Hammarlund, M., S. Watanabe, K. Schuske, and E.M. Jorgensen. 2008. CAPS and syntaxin dock dense core vesicles to the plasma membrane in neurons. J Cell Biol. 180:483-491.

Hanson, P.I., J.E. Heuser, and R. Jahn. 1997. Neurotransmitter release - four years of SNARE complexes. Curr Opin Neurobiol. 7:310-315.

Harata, N., T.A. Ryan, S.J. Smith, J. Buchanan, and R.W. Tsien. 2001. Visualizing recycling synaptic vesicles in hippocampal neurons by FM 1-43 photoconversion. Proc Natl Acad Sci U S A. 98:12748-12753.

Hata, Y., C.A. Slaughter, and T.C. Sudhof. 1993. Synaptic vesicle fusion complex contains unc-18 homologue bound to syntaxin. Nature. 366:347-351. 
Hawkins, K.L., R.V. Lloyd, and K.A. Toy. 1989. Immunohistochemical localization of chromogranin A in normal tissues from laboratory animals. Veterinary pathology. 26:488-498.

Higashio, H., N. Nishimura, H. Ishizaki, J. Miyoshi, S. Orita, A. Sakane, and T. Sasaki. 2008. Doc2 alpha and Munc13-4 regulate $\mathrm{Ca}(2+)$-dependent secretory lysosome exocytosis in mast cells. J Immunol. 180:4774-4784.

Holgert, H., A. Dagerlind, and T. Hokfelt. 1996. Phenotype of intraadrenal ganglion neurons during postnatal development in rat. The Journal of comparative neurology. 371:603-620.

Honigmann, A., G. van den Bogaart, E. Iraheta, H.J. Risselada, D. Milovanovic, V. Mueller, S. Mullar, U. Diederichsen, D. Fasshauer, H. Grubmuller, S.W. Hell, C. Eggeling, K. Kuhnel, and R. Jahn. 2013. Phosphatidylinositol 4,5-bisphosphate clusters act as molecular beacons for vesicle recruitment. Nat Struct Mol Biol. 20:679-686.

Horrigan, F.T., and R.J. Bookman. 1994. Releasable pools and the kinetics of exocytosis in adrenal chromaffin cells. Neuron. 13:1119-1129.

Jahn, R., and D. Fasshauer. 2012. Molecular machines governing exocytosis of synaptic vesicles. Nature. 490:201-207.

James, D.J., C. Khodthong, J.A. Kowalchyk, and T.F. Martin. 2008. Phosphatidylinositol 4,5-bisphosphate regulates SNARE-dependent membrane fusion. J Cell Biol. 182:355366.

James, D.J., J. Kowalchyk, N. Daily, M. Petrie, and T.F. Martin. 2009. CAPS drives transSNARE complex formation and membrane fusion through syntaxin interactions. Proc Natl Acad Sci U S A. 106:17308-17313.

James, D.J., and T.F. Martin. 2013. CAPS and Munc13: CATCHRs that SNARE Vesicles. Frontiers in endocrinology. 4:187.

Jockusch, W.J., D. Speidel, A. Sigler, J.B. Sorensen, F. Varoqueaux, J.S. Rhee, and N. Brose. 2007. CAPS-1 and CAPS-2 are essential synaptic vesicle priming proteins. Cell. 131:796808.

Junge, H.J., J.S. Rhee, O. Jahn, F. Varoqueaux, J. Spiess, M.N. Waxham, C. Rosenmund, and N. Brose. 2004. Calmodulin and Munc13 form a Ca2+ sensor/effector complex that controls short-term synaptic plasticity. Cell. 118:389-401.

Kabachinski, G., M. Yamaga, D.M. Kielar-Grevstad, S. Bruinsma, and T.F. Martin. 2013. CAPS and Munc13 utilize distinct PIP2-linked mechanisms to promote vesicle exocytosis. Mol Biol Cell.

Kaeser, P.S. 2011. Pushing synaptic vesicles over the RIM. Cellular logistics. 1:106-110.

Kaeser, P.S., L. Deng, Y. Wang, I. Dulubova, X. Liu, J. Rizo, and T.C. Sudhof. 2011. RIM proteins tether $\mathrm{Ca} 2+$ channels to presynaptic active zones via a direct PDZ-domain interaction. Cell. 144:282-295. 
Kalla, S., M. Stern, J. Basu, F. Varoqueaux, K. Reim, C. Rosenmund, N.E. Ziv, and N. Brose. 2006. Molecular dynamics of a presynaptic active zone protein studied in Munc13-1enhanced yellow fluorescent protein knock-in mutant mice. J Neurosci. 26:1305413066.

Kamin, D., M.A. Lauterbach, V. Westphal, J. Keller, A. Schonle, S.W. Hell, and S.O. Rizzoli. 2010. High- and low-mobility stages in the synaptic vesicle cycle. Biophys J. 99:675-684.

Kandel, E.R., J.H. Schwartz, T.M. Jessell, S.A. Siegelbaum, and A.J. Hudspeth. 2013. Principles of Neural Science. The McGraw-Hill Companies, United States of America.

Kang, L., Z. He, P. Xu, J. Fan, A. Betz, N. Brose, and T. Xu. 2006. Munc13-1 is required for the sustained release of insulin from pancreatic beta cells. Cell metabolism. 3:463-468.

Kasai, K., M. Ohara-Imaizumi, N. Takahashi, S. Mizutani, S. Zhao, T. Kikuta, H. Kasai, S. Nagamatsu, H. Gomi, and T. Izumi. 2005. Rab27a mediates the tight docking of insulin granules onto the plasma membrane during glucose stimulation. J Clin Invest. 115:388396.

Khodthong, C., G. Kabachinski, D.J. James, and T.F. Martin. 2011. Munc13 homology domain-1 in CAPS/UNC31 mediates SNARE binding required for priming vesicle exocytosis. Cell metabolism. 14:254-263.

Khvotchev, M., I. Dulubova, J. Sun, H. Dai, J. Rizo, and T.C. Sudhof. 2007. Dual modes of Munc18-1/SNARE interactions are coupled by functionally critical binding to syntaxin-1 N terminus. J Neurosci. 27:12147-12155.

Kim, S.H., and T.A. Ryan. 2010. CDK5 serves as a major control point in neurotransmitter release. Neuron. 67:797-809.

Kim, T., J.H. Tao-Cheng, L.E. Eiden, and Y.P. Loh. 2001. Chromogranin A, an "on/off" switch controlling dense-core secretory granule biogenesis. Cell. 106:499-509.

Kloepper, T.H., C.N. Kienle, and D. Fasshauer. 2007. An elaborate classification of SNARE proteins sheds light on the conservation of the eukaryotic endomembrane system. $\mathrm{Mol}$ Biol Cell. 18:3463-3471.

Klumperman, J., R. Kuliawat, J.M. Griffith, H.J. Geuze, and P. Arvan. 1998. Mannose 6phosphate receptors are sorted from immature secretory granules via adaptor protein AP-1, clathrin, and syntaxin 6-positive vesicles. J Cell Biol. 141:359-371.

Koch, H., K. Hofmann, and N. Brose. 2000. Definition of Munc13-homology-domains and characterization of a novel ubiquitously expressed Munc13 isoform. Biochem J. 349:247-253.

Kozlovsky, Y., and M.M. Kozlov. 2002. Stalk model of membrane fusion: solution of energy crisis. Biophys J. 82:882-895.

Kriebel, M.E., R. Hanna, and C. Muniak. 1986. Synaptic vesicle diameters and synaptic cleft widths at the mouse diaphragm in neonates and adults. Brain Res. 392:19-29. 
Kuromi, H., and Y. Kidokoro. 2005. Exocytosis and endocytosis of synaptic vesicles and functional roles of vesicle pools: lessons from the Drosophila neuromuscular junction. Neuroscientist. 11:138-147.

Lang, T., D. Bruns, D. Wenzel, D. Riedel, P. Holroyd, C. Thiele, and R. Jahn. 2001. SNAREs are concentrated in cholesterol-dependent clusters that define docking and fusion sites for exocytosis. Embo j. 20:2202-2213.

Lawrence, G.W., P. Foran, N. Mohammed, B.R. DasGupta, and J.O. Dolly. 1997. Importance of two adjacent C-terminal sequences of SNAP-25 in exocytosis from intact and permeabilized chromaffin cells revealed by inhibition with botulinum neurotoxins A and E. Biochemistry. 36:3061-3067.

Lee, J.S., W.K. Ho, and S.H. Lee. 2012. Actin-dependent rapid recruitment of reluctant synaptic vesicles into a fast-releasing vesicle pool. Proc Natl Acad Sci U S A. 109:E765774.

Lee, J.S., W.K. Ho, E. Neher, and S.H. Lee. 2013. Superpriming of synaptic vesicles after their recruitment to the readily releasable pool. Proc Natl Acad Sci U S A. 110:1507915084.

Lerman, J.C., J. Robblee, R. Fairman, and F.M. Hughson. 2000. Structural analysis of the neuronal SNARE protein syntaxin-1A. Biochemistry. 39:8470-8479.

Li, F., F. Pincet, E. Perez, W.S. Eng, T.J. Melia, J.E. Rothman, and D. Tareste. 2007. Energetics and dynamics of SNAREpin folding across lipid bilayers. Nat Struct Mol Biol. 14:890-896.

Li, W., C. Ma, R. Guan, Y. Xu, D.R. Tomchick, and J. Rizo. 2011. The crystal structure of a Munc13 C-terminal module exhibits a remarkable similarity to vesicle tethering factors. Structure (London, England : 1993). 19:1443-1455.

Li, Z., J. Burrone, W.J. Tyler, K.N. Hartman, D.F. Albeanu, and V.N. Murthy. 2005. Synaptic vesicle recycling studied in transgenic mice expressing synaptopHluorin. Proc Natl Acad Sci U S A. 102:6131-6136.

Lindau, M., and E. Neher. 1988. Patch-clamp techniques for time-resolved capacitance measurements in single cells. Pflugers Arch. 411:137-146.

Lipstein, N., T. Sakaba, B.H. Cooper, K.H. Lin, N. Strenzke, U. Ashery, J.S. Rhee, H. Taschenberger, E. Neher, and N. Brose. 2013. Dynamic control of synaptic vesicle replenishment and short-term plasticity by $\mathrm{Ca}(2+)$-calmodulin-Munc13-1 signaling. Neuron. 79:82-96.

Lipstein, N., S. Schaks, K. Dimova, S. Kalkhof, C. Ihling, K. Kolbel, U. Ashery, J. Rhee, N. Brose, A. Sinz, and O. Jahn. 2012. Nonconserved $\mathrm{Ca}(2+) /$ calmodulin binding sites in Munc13s differentially control synaptic short-term plasticity. Mol Cell Biol. 32:46284641. 
Littleton, J.T., R.J. Barnard, S.A. Titus, J. Slind, E.R. Chapman, and B. Ganetzky. 2001. SNARE-complex disassembly by NSF follows synaptic-vesicle fusion. Proc Natl Acad Sci U S A. 98:12233-12238.

Liu, W., V. Montana, V. Parpura, and U. Mohideen. 2009. Single Molecule Measurements of Interaction Free Energies Between the Proteins Within Binary and Ternary SNARE Complexes. J Nanoneurosci. 1:120-129.

Liu, Y., C. Schirra, L. Edelmann, U. Matti, J. Rhee, D. Hof, D. Bruns, N. Brose, H. Rieger, D.R. Stevens, and J. Rettig. 2010. Two distinct secretory vesicle-priming steps in adrenal chromaffin cells. J Cell Biol. 190:1067-1077.

Liu, Y., C. Schirra, D.R. Stevens, U. Matti, D. Speidel, D. Hof, D. Bruns, N. Brose, and J. Rettig. 2008. CAPS facilitates filling of the rapidly releasable pool of large dense-core vesicles. J Neurosci. 28:5594-5601.

Ma, C., W. Li, Y. Xu, and J. Rizo. 2011. Munc13 mediates the transition from the closed syntaxin-Munc18 complex to the SNARE complex. Nat Struct Mol Biol. 18:542-549.

Madison, J.M., S. Nurrish, and J.M. Kaplan. 2005. UNC-13 interaction with syntaxin is required for synaptic transmission. Curr Biol. 15:2236-2242.

Maeder, C.I., A. San-Miguel, E.Y. Wu, H. Lu, and K. Shen. 2013. In Vivo Neuron-Wide Analysis of Synaptic Vesicle Precursor Trafficking. Traffic.

Maniatis, T. 1982. Molecular cloning : a laboratory manual / T. Maniatis, E.F. Fritsch, J. Sambrook. Cold Spring Harbor Laboratory, Cold Spring Harbor, N.Y.

McEwen, J.M., J.M. Madison, M. Dybbs, and J.M. Kaplan. 2006. Antagonistic regulation of synaptic vesicle priming by Tomosyn and UNC-13. Neuron. 51:303-315.

McMahon, H.T., P. Foran, J.O. Dolly, M. Verhage, V.M. Wiegant, and D.G. Nicholls. 1992. Tetanus toxin and botulinum toxins type A and B inhibit glutamate, gammaaminobutyric acid, aspartate, and met-enkephalin release from synaptosomes. Clues to the locus of action.J Biol Chem. 267:21338-21343.

Menager, M.M., G. Menasche, M. Romao, P. Knapnougel, C.H. Ho, M. Garfa, G. Raposo, J. Feldmann, A. Fischer, and G. de Saint Basile. 2007. Secretory cytotoxic granule maturation and exocytosis require the effector protein hMunc13-4. Nat Immunol. 8:257267.

Milosevic, I., J.B. Sorensen, T. Lang, M. Krauss, G. Nagy, V. Haucke, R. Jahn, and E. Neher. 2005. Plasmalemmal phosphatidylinositol-4,5-bisphosphate level regulates the releasable vesicle pool size in chromaffin cells. J Neurosci. 25:2557-2565.

Misler, S. 2009. Unifying concepts in stimulus-secretion coupling in endocrine cells and some implications for therapeutics. Advances in physiology education. 33:175-186.

Misura, K.M., L.C. Gonzalez, Jr., A.P. May, R.H. Scheller, and W.I. Weis. 2001a. Crystal structure and biophysical properties of a complex between the N-terminal SNARE region of SNAP25 and syntaxin 1a.J Biol Chem. 276:41301-41309. 
Misura, K.M., R.H. Scheller, and W.I. Weis. 2000. Three-dimensional structure of the neuronal-Sec1-syntaxin 1a complex. Nature. 404:355-362.

Misura, K.M., R.H. Scheller, and W.I. Weis. 2001b. Self-association of the H3 region of syntaxin 1A. Implications for intermediates in SNARE complex assembly. J Biol Chem. 276:13273-13282.

Mohrmann, R., H. de Wit, M. Verhage, E. Neher, and J.B. Sorensen. 2010. Fast vesicle fusion in living cells requires at least three SNARE complexes. Science. 330:502-505.

Mohrmann, R., and J.B. Sorensen. 2012. SNARE requirements en route to exocytosis: from many to few. Journal of molecular neuroscience : MN. 48:387-394.

Moser, T., and E. Neher. 1997. Rapid exocytosis in single chromaffin cells recorded from mouse adrenal slices. J Neurosci. 17:2314-2323.

Murthy, V.N., and C.F. Stevens. 1999. Reversal of synaptic vesicle docking at central synapses. Nat Neurosci. 2:503-507.

Nagy, G., U. Matti, R.B. Nehring, T. Binz, J. Rettig, E. Neher, and J.B. Sorensen. 2002. Protein kinase C-dependent phosphorylation of synaptosome-associated protein of 25 kDa at Ser187 potentiates vesicle recruitment. J Neurosci. 22:9278-9286.

Nagy, G., I. Milosevic, D. Fasshauer, E.M. Muller, B.L. de Groot, T. Lang, M.C. Wilson, and J.B. Sorensen. 2005. Alternative splicing of SNAP-25 regulates secretion through nonconservative substitutions in the SNARE domain. Mol Biol Cell. 16:5675-5685.

Nagy, G., I. Milosevic, R. Mohrmann, K. Wiederhold, A.M. Walter, and J.B. Sorensen. 2008. The SNAP-25 linker as an adaptation toward fast exocytosis. Mol Biol Cell. 19:37693781.

Naraghi, M., T.H. Muller, and E. Neher. 1998. Two-dimensional determination of the cellular Ca2+ binding in bovine chromaffin cells. Biophys J. 75:1635-1647.

Neeft, M., M. Wieffer, A.S. de Jong, G. Negroiu, C.H. Metz, A. van Loon, J. Griffith, J. Krijgsveld, N. Wulffraat, H. Koch, A.J. Heck, N. Brose, M. Kleijmeer, and P. van der Sluijs. 2005. Munc13-4 is an effector of rab27a and controls secretion of lysosomes in hematopoietic cells. Mol Biol Cell. 16:731-741.

Neher, E. 1998. Vesicle pools and Ca2+ microdomains: new tools for understanding their roles in neurotransmitter release. Neuron. 20:389-399.

Neher, E. 2006. A comparison between exocytic control mechanisms in adrenal chromaffin cells and a glutamatergic synapse. Pflugers Arch. 453:261-268.

Neher, E., and A. Marty. 1982. Discrete changes of cell membrane capacitance observed under conditions of enhanced secretion in bovine adrenal chromaffin cells. Proc Natl Acad Sci U S A. 79:6712-6716.

Ohara-Imaizumi, M., T. Fujiwara, Y. Nakamichi, T. Okamura, Y. Akimoto, J. Kawai, S. Matsushima, H. Kawakami, T. Watanabe, K. Akagawa, and S. Nagamatsu. 2007. Imaging 
analysis reveals mechanistic differences between first- and second-phase insulin exocytosis. J Cell Biol. 177:695-705.

Palmer, R.E., S.B. Lee, J.C. Wong, P.A. Reynolds, H. Zhang, V. Truong, J.D. Oliner, W.L. Gerald, and D.A. Haber. 2002. Induction of BAIAP3 by the EWS-WT1 chimeric fusion implicates regulated exocytosis in tumorigenesis. Cancer Cell. 2:497-505.

Pardo-Bellver, C., B. Cadiz-Moretti, A. Novejarque, F. Martinez-Garcia, and E. Lanuza. 2012. Differential efferent projections of the anterior, posteroventral, and posterodorsal subdivisions of the medial amygdala in mice. Frontiers in neuroanatomy. 6:33.

Pei, J., C. Ma, J. Rizo, and N.V. Grishin. 2009. Remote homology between Munc13 MUN domain and vesicle tethering complexes. J Mol Biol. 391:509-517.

Perrais, D., I.C. Kleppe, J.W. Taraska, and W. Almers. 2004. Recapture after exocytosis causes differential retention of protein in granules of bovine chromaffin cells. J Physiol. 560:413-428.

Pinheiro, P.S., H. de Wit, A.M. Walter, A.J. Groffen, M. Verhage, and J.B. Sorensen. 2013. Doc $2 b$ synchronizes secretion from chromaffin cells by stimulating fast and inhibiting sustained release. J Neurosci. 33:16459-16470.

Pivot-Pajot, C., F. Varoqueaux, G. de Saint Basile, and S.G. Bourgoin. 2008. Munc13-4 regulates granule secretion in human neutrophils. J Immunol. 180:6786-6797.

Pobbati, A.V., A. Stein, and D. Fasshauer. 2006. N- to C-terminal SNARE complex assembly promotes rapid membrane fusion. Science. 313:673-676.

Rhee, J.S., A. Betz, S. Pyott, K. Reim, F. Varoqueaux, I. Augustin, D. Hesse, T.C. Sudhof, M. Takahashi, C. Rosenmund, and N. Brose. 2002. Beta phorbol ester- and diacylglycerolinduced augmentation of transmitter release is mediated by Munc13s and not by PKCs. Cell. 108:121-133.

Richmond, J.E., W.S. Davis, and E.M. Jorgensen. 1999. UNC-13 is required for synaptic vesicle fusion in C. elegans. Nat Neurosci. 2:959-964.

Rickman, C., C.N. Medine, A.R. Dun, D.J. Moulton, O. Mandula, N.D. Halemani, S.O. Rizzoli, L.H. Chamberlain, and R.R. Duncan. 2010. t-SNARE protein conformations patterned by the lipid microenvironment. J Biol Chem. 285:13535-13541.

Rizo, J., and T.C. Sudhof. 2012. The membrane fusion enigma: SNAREs, Sec1/Munc18 proteins, and their accomplices--guilty as charged? Annu Rev Cell Dev Biol. 28:279-308.

Rizzoli, S.O., and W.J. Betz. 2004. The structural organization of the readily releasable pool of synaptic vesicles. Science. 303:2037-2039.

Rizzoli, S.O., and W.J. Betz. 2005. Synaptic vesicle pools. Nat Rev Neurosci. 6:57-69.

Rosenmund, C., A. Sigler, I. Augustin, K. Reim, N. Brose, and J.S. Rhee. 2002. Differential control of vesicle priming and short-term plasticity by Munc13 isoforms. Neuron. 33:411-424. 
Rosenmund, C., and C.F. Stevens. 1996. Definition of the readily releasable pool of vesicles at hippocampal synapses. Neuron. 16:1197-1207.

Rothman, J.E. 1994. Mechanisms of intracellular protein transport. Nature. 372:55-63.

Sabatini, B.L., and W.G. Regehr. 1996. Timing of neurotransmission at fast synapses in the mammalian brain. Nature. 384:170-172.

Saheki, Y., and P. De Camilli. 2012. Synaptic vesicle endocytosis. Cold Spring Harb Perspect Biol. 4:a005645.

Sajdyk, T.J., A. Shekhar, and D.R. Gehlert. 2004. Interactions between NPY and CRF in the amygdala to regulate emotionality. Neuropeptides. 38:225-234.

Santos, M.S., H. Li, and S.M. Voglmaier. 2009. Synaptic vesicle protein trafficking at the glutamate synapse. Neuroscience. 158:189-203.

Schikorski, T., and C.F. Stevens. 1997. Quantitative ultrastructural analysis of hippocampal excitatory synapses. J Neurosci. 17:5858-5867.

Schonn, J.S., A. Maximov, Y. Lao, T.C. Sudhof, and J.B. Sorensen. 2008. Synaptotagmin-1 and -7 are functionally overlapping $\mathrm{Ca} 2+$ sensors for exocytosis in adrenal chromaffin cells. Proc Natl Acad Sci U S A. 105:3998-4003.

Schonn, J.S., J.R. van Weering, R. Mohrmann, O.M. Schluter, T.C. Sudhof, H. de Wit, M. Verhage, and J.B. Sorensen. 2010. Rab3 proteins involved in vesicle biogenesis and priming in embryonic mouse chromaffin cells. Traffic. 11:1415-1428.

Shen, J., S.S. Rathore, L. Khandan, and J.E. Rothman. 2010. SNARE bundle and syntaxin Npeptide constitute a minimal complement for Munc18-1 activation of membrane fusion. J Cell Biol. 190:55-63.

Sheu, L., E.A. Pasyk, J. Ji, X. Huang, X. Gao, F. Varoqueaux, N. Brose, and H.Y. Gaisano. 2003. Regulation of insulin exocytosis by Munc13-1. J Biol Chem. 278:27556-27563.

Shin, O.H., J. Lu, J.S. Rhee, D.R. Tomchick, Z.P. Pang, S.M. Wojcik, M. Camacho-Perez, N. Brose, M. Machius, J. Rizo, C. Rosenmund, and T.C. Sudhof. 2010. Munc13 C2B domain is an activity-dependent Ca2+ regulator of synaptic exocytosis. Nat Struct Mol Biol. 17:280-288.

Shirakawa, R., T. Higashi, A. Tabuchi, A. Yoshioka, H. Nishioka, M. Fukuda, T. Kita, and H. Horiuchi. 2004. Munc13-4 is a GTP-Rab27-binding protein regulating dense core granule secretion in platelets. J Biol Chem. 279:10730-10737.

Shiratsuchi, T., K. Oda, H. Nishimori, M. Suzuki, E. Takahashi, T. Tokino, and Y. Nakamura. 1998. Cloning and characterization of BAP3 (BAI-associated protein 3), a C2 domain-containing protein that interacts with BAI1. Biochem Biophys Res Commun. 251:158-165. 
Sieber, J.J., K.I. Willig, C. Kutzner, C. Gerding-Reimers, B. Harke, G. Donnert, B. Rammner, C. Eggeling, S.W. Hell, H. Grubmuller, and T. Lang. 2007. Anatomy and dynamics of a supramolecular membrane protein cluster. Science. 317:1072-1076.

Sieburth, D., J.M. Madison, and J.M. Kaplan. 2007. PKC-1 regulates secretion of neuropeptides. Nat Neurosci. 10:49-57.

Siksou, L., F. Varoqueaux, O. Pascual, A. Triller, N. Brose, and S. Marty. 2009. A common molecular basis for membrane docking and functional priming of synaptic vesicles. Eur J Neurosci. 30:49-56.

Sinha, R., S. Ahmed, R. Jahn, and J. Klingauf. 2011. Two synaptobrevin molecules are sufficient for vesicle fusion in central nervous system synapses. Proc Natl Acad Sci U S A. 108:14318-14323.

Smith, C., T. Moser, T. Xu, and E. Neher. 1998. Cytosolic Ca2+ acts by two separate pathways to modulate the supply of release-competent vesicles in chromaffin cells. Neuron. 20:1243-1253.

Smith, J.E., and T.S. Reese. 1980. Use of aldehyde fixatives to determine the rate of synaptic transmitter release. J Exp Biol. 89:19-29.

Sollner, T., M.K. Bennett, S.W. Whiteheart, R.H. Scheller, and J.E. Rothman. 1993a. A protein assembly-disassembly pathway in vitro that may correspond to sequential steps of synaptic vesicle docking, activation, and fusion. Cell. 75:409-418.

Sollner, T., S.W. Whiteheart, M. Brunner, H. Erdjument-Bromage, S. Geromanos, P. Tempst, and J.E. Rothman. 1993b. SNAP receptors implicated in vesicle targeting and fusion. Nature. 362:318-324.

Sorensen, J.B. 2004. Formation, stabilisation and fusion of the readily releasable pool of secretory vesicles. Pflugers Arch. 448:347-362.

Sorensen, J.B., G. Nagy, F. Varoqueaux, R.B. Nehring, N. Brose, M.C. Wilson, and E. Neher. 2003. Differential control of the releasable vesicle pools by SNAP-25 splice variants and SNAP-23. Cell. 114:75-86.

Speese, S., M. Petrie, K. Schuske, M. Ailion, K. Ann, K. Iwasaki, E.M. Jorgensen, and T.F. Martin. 2007. UNC-31 (CAPS) is required for dense-core vesicle but not synaptic vesicle exocytosis in Caenorhabditis elegans. J Neurosci. 27:6150-6162.

Speidel, D., C.E. Bruederle, C. Enk, T. Voets, F. Varoqueaux, K. Reim, U. Becherer, F. Fornai, S. Ruggieri, Y. Holighaus, E. Weihe, D. Bruns, N. Brose, and J. Rettig. 2005. CAPS1 regulates catecholamine loading of large dense-core vesicles. Neuron. 46:75-88.

Speidel, D., F. Varoqueaux, C. Enk, M. Nojiri, R.N. Grishanin, T.F. Martin, K. Hofmann, N. Brose, and K. Reim. 2003. A family of Ca2+-dependent activator proteins for secretion: comparative analysis of structure, expression, localization, and function.J Biol Chem. 278:52802-52809. 
Stephenson, J.R., K.J. Paavola, S.A. Schaefer, B. Kaur, E.G. Van Meir, and R.A. Hall. 2013. Brain-specific angiogenesis inhibitor-1 signaling, regulation, and enrichment in the postsynaptic density. J Biol Chem. 288:22248-22256.

Stevens, C.F., and J.M. Sullivan. 1998. Regulation of the readily releasable vesicle pool by protein kinase C. Neuron. 21:885-893.

Stevens, C.F., and J.H. Williams. 2007. Discharge of the readily releasable pool with action potentials at hippocampal synapses. J Neurophysiol. 98:3221-3229.

Stevens, D.R., Z.X. Wu, U. Matti, H.J. Junge, C. Schirra, U. Becherer, S.M. Wojcik, N. Brose, and J. Rettig. 2005. Identification of the minimal protein domain required for priming activity of Munc13-1. Curr Biol. 15:2243-2248.

Toonen, R.F., K.J. de Vries, R. Zalm, T.C. Sudhof, and M. Verhage. 2005. Munc18-1 stabilizes syntaxin 1, but is not essential for syntaxin 1 targeting and SNARE complex formation.J Neurochem. 93:1393-1400.

Toonen, R.F., O. Kochubey, H. de Wit, A. Gulyas-Kovacs, B. Konijnenburg, J.B. Sorensen, J. Klingauf, and M. Verhage. 2006a. Dissecting docking and tethering of secretory vesicles at the target membrane. EMBO J. 25:3725-3737.

Toonen, R.F., K. Wierda, M.S. Sons, H. de Wit, L.N. Cornelisse, A. Brussaard, J.J. Plomp, and M. Verhage. 2006b. Munc18-1 expression levels control synapse recovery by regulating readily releasable pool size. Proc Natl Acad Sci U S A. 103:18332-18337.

Tsuboi, T., and M. Fukuda. 2006. Rab3A and Rab27A cooperatively regulate the docking step of dense-core vesicle exocytosis in PC12 cells. J Cell Sci. 119:2196-2203.

van de Bospoort, R., M. Farina, S.K. Schmitz, A. de Jong, H. de Wit, M. Verhage, and R.F. Toonen. 2012. Munc13 controls the location and efficiency of dense-core vesicle release in neurons. J Cell Biol. 199:883-891.

van den Bogaart, G., M.G. Holt, G. Bunt, D. Riedel, F.S. Wouters, and R. Jahn. 2010. One SNARE complex is sufficient for membrane fusion. Nat Struct Mol Biol. 17:358-364.

van den Bogaart, G., K. Meyenberg, H.J. Risselada, H. Amin, K.I. Willig, B.E. Hubrich, M. Dier, S.W. Hell, H. Grubmuller, U. Diederichsen, and R. Jahn. 2011. Membrane protein sequestering by ionic protein-lipid interactions. Nature. 479:552-555.

van Weering, J.R., R.F. Toonen, and M. Verhage. 2007. The role of Rab3a in secretory vesicle docking requires association/dissociation of guanidine phosphates and Munc181. PLoS One. 2:e616.

Varoqueaux, F., A. Sigler, J.S. Rhee, N. Brose, C. Enk, K. Reim, and C. Rosenmund. 2002. Total arrest of spontaneous and evoked synaptic transmission but normal synaptogenesis in the absence of Munc13-mediated vesicle priming. Proc Natl Acad Sci U S A. 99:9037-9042. 
Varoqueaux, F., M.S. Sons, J.J. Plomp, and N. Brose. 2005. Aberrant morphology and residual transmitter release at the Munc13-deficient mouse neuromuscular synapse. Mol Cell Biol. 25:5973-5984.

Verhage, M., A.S. Maia, J.J. Plomp, A.B. Brussaard, J.H. Heeroma, H. Vermeer, R.F. Toonen, R.E. Hammer, T.K. van den Berg, M. Missler, H.J. Geuze, and T.C. Sudhof. 2000. Synaptic assembly of the brain in the absence of neurotransmitter secretion. Science. 287:864869.

Verhage, M., and J.B. Sorensen. 2008. Vesicle docking in regulated exocytosis. Traffic. 9:1414-1424.

Voets, T. 2000. Dissection of three Ca2+-dependent steps leading to secretion in chromaffin cells from mouse adrenal slices. Neuron. 28:537-545.

Voets, T., E. Neher, and T. Moser. 1999. Mechanisms underlying phasic and sustained secretion in chromaffin cells from mouse adrenal slices. Neuron. 23:607-615.

Voets, T., R.F. Toonen, E.C. Brian, H. de Wit, T. Moser, J. Rettig, T.C. Sudhof, E. Neher, and M. Verhage. 2001. Munc18-1 promotes large dense-core vesicle docking. Neuron.

31:581-591.

Walter, A.M., P.S. Pinheiro, M. Verhage, and J.B. Sorensen. 2013. A sequential vesicle pool model with a single release sensor and a ca(2+)-dependent priming catalyst effectively explains $\mathrm{ca}(2+)$-dependent properties of neurosecretion. PLoS Comput Biol. 9:e1003362.

Walter, A.M., K. Wiederhold, D. Bruns, D. Fasshauer, and J.B. Sorensen. 2010. Synaptobrevin N-terminally bound to syntaxin-SNAP-25 defines the primed vesicle state in regulated exocytosis. J Cell Biol. 188:401-413.

Wang, Q., M. Wang, and M.D. Whim. 2013. Neuropeptide y gates a stress-induced, longlasting plasticity in the sympathetic nervous system. J Neurosci. 33:12705-12717.

Weimer, R.M., J.E. Richmond, W.S. Davis, G. Hadwiger, M.L. Nonet, and E.M. Jorgensen. 2003. Defects in synaptic vesicle docking in unc-18 mutants. Nat Neurosci. 6:1023-1030.

Whim, M.D. 2006. Near simultaneous release of classical and peptide cotransmitters from chromaffin cells. J Neurosci. 26:6637-6642.

Wiederhold, K., and D. Fasshauer. 2009. Is assembly of the SNARE complex enough to fuel membrane fusion? J Biol Chem. 284:13143-13152.

Wojcik, S.M., and N. Brose. 2007. Regulation of membrane fusion in synaptic excitationsecretion coupling: speed and accuracy matter. Neuron. 55:11-24.

Wojcik, S.M., M. Tantra, B. Stepniak, K.N. Man, K. Muller-Ribbe, M. Begemann, A. Ju, S. Papiol, A. Ronnenberg, A. Gurvich, Y. Shin, I. Augustin, N. Brose, and H. Ehrenreich. 2013. Genetic markers of a Munc13 protein family member, BAIAP3, are gender specifically associated with anxiety and benzodiazepine abuse in mice and humans. Mol Med. 19:135-148. 
Wu, Y., Y. Gu, M.K. Morphew, J. Yao, F.L. Yeh, M. Dong, and E.R. Chapman. 2012. All three components of the neuronal SNARE complex contribute to secretory vesicle docking. $J$ Cell Biol. 198:323-330.

Xu, T., T. Binz, H. Niemann, and E. Neher. 1998. Multiple kinetic components of exocytosis distinguished by neurotoxin sensitivity. Nat Neurosci. 1:192-200.

Xu, T., and P. Xu. 2008. Differential Regulation of Small Clear Vesicles and Large DenseCore Vesicles. Humana Press.

Yim, Y.I., T. Sun, L.G. Wu, A. Raimondi, P. De Camilli, E. Eisenberg, and L.E. Greene. 2010. Endocytosis and clathrin-uncoating defects at synapses of auxilin knockout mice. Proc Natl Acad Sci U S A. 107:4412-4417.

Zahn, T.R., J.K. Angleson, M.A. MacMorris, E. Domke, J.F. Hutton, C. Schwartz, and J.C. Hutton. 2004. Dense core vesicle dynamics in Caenorhabditis elegans neurons and the role of kinesin UNC-104. Traffic. 5:544-559.

Zhou, K.M., Y.M. Dong, Q. Ge, D. Zhu, W. Zhou, X.G. Lin, T. Liang, Z.X. Wu, and T. Xu. 2007. PKA activation bypasses the requirement for UNC-31 in the docking of dense core vesicles from C. elegans neurons. Neuron. 56:657-669.

Zhou, P., Z.P. Pang, X. Yang, Y. Zhang, C. Rosenmund, T. Bacaj, and T.C. Sudhof. 2013. Syntaxin-1 N-peptide and Habc-domain perform distinct essential functions in synaptic vesicle fusion. EMBO J. 32:159-171.

Zhou, Z., and S. Misler. 1995. Action potential-induced quantal secretion of catecholamines from rat adrenal chromaffin cells. J Biol Chem. 270:3498-3505.

Zikich, D., A. Mezer, F. Varoqueaux, A. Sheinin, H.J. Junge, E. Nachliel, R. Melamed, N. Brose, M. Gutman, and U. Ashery. 2008. Vesicle priming and recruitment by ubMunc132 are differentially regulated by calcium and calmodulin. J Neurosci. 28:1949-1960.

Zucker, R.S., and W.G. Regehr. 2002. Short-term synaptic plasticity. Annu Rev Physiol. 64:355-405. 
Date of Birth: $13^{\text {th }}$ November 1982

MAN Kwun-nok Mimi

Place of Birth: Hong Kong

Nationality: Hong Kong

Address: $\quad$ Zimmer 91, Annastrasse 17, 37075 Göttingen, Germany.

Phone: $\quad+49-(0) 17661770810$

E-mail: $\quad$ man@em.mpg.de; sasha.sashimi@gmail.com

\section{Education and Professional Experience}

$\begin{array}{ll}\text { 05/2014-present } & \begin{array}{l}\text { Postdoctoral scientist } \\ \text { Max Planck Institute of Experimental Medicine, Göttingen, Germany. }\end{array} \\ & \text { Doctor rerum naturalium (magna cum laude) } \\ \text { Georg-August-Universität Göttingen, Göttingen, Germany; } \\ \text { Max Planck Institute of Experimental Medicine, Göttingen, Germany. } \\ \text { Thesis title: Role of Munc13 Isoforms in Regulating Large Dense } \\ \text { Core Vesicle Exocytosis in Chromaffin Cells }\end{array}$

01/2007-07/2008 Research Assistant, Dept of Obstetrics and Gynaecology, Chinese University of Hong Kong.

Expression profiling and target prediction of microRNAs in cervical intraepithelial neoplasia and cervical squamous cell carcinoma

09/2004-12/2006

Master of Philosophy

Department of Zoology, University of Hong Kong.

Thesis title: Functional Implications of Cytoglobin, a Novel Protein, in Liver Fibrosis

09/2001-06/2004 Bachelor of Science (Second Class Upper Hons)

in Animal and Plant Biotechnology, University of Hong Kong.

\section{Publications and Presentations}

\section{Paper publications (An asterisk indicates co-authorship.)}

1. Man K.N., Imig, C., Walter A.M., Pinheiro P.S., Stevens D.R., Rettig J., Sørensen J.B., Cooper B.H., Brose N., Wojcik, S.M. Identification of a Munc13-sensitive step in chromaffin cell large dense-core vesicle exocytosis. Revision submitted at eLife.

2. Wojcik S.M., Tantra M., Stepniak B., Man K.N., Müller-Ribbe K., Begemann M., Ju A., Papiol S., Ronnenberg A., Gurvich A., Shin Y., Augustin I., Brose N., Ehrenreich H. (2013) Genetic markers of a Munc13 protein family member, BAIAP3, are gender specifically associated with anxiety and benzodiazepine abuse in mice and humans. Mol. Med. 19, 13548.

3. Cheung T.H.*, Man K.N.*, Yu M.Y., Yim S.F., Siu N.S., Lo K.W., Doran G., Wong R.R., Wang V.W., Smith D.I., Worley M.J. Jr, Berkowitz R.S., Chung T.K., Wong Y.F. (2012) Dysregulated microRNAs in the pathogenesis and progression of cervical neoplasm. Cell Cycle 11(15), 2876-84.

4. Burgalossi A., Jung S., Man K.N., Nair R., Jockusch W.J., Wojcik S.M., Brose N., Rhee J.S. (2012) Analysis of neurotransmitter release mechanisms by photolysis of caged $\mathrm{Ca}^{2+}$ in an autaptic neuron culture system. Nat. Protoc. 7(7), 1351-65.

5. Man, K.N.M., Philipsen, S., Tan-Un, K.C. (2008) Localization and Expression Pattern of Cytoglobin in carbon tetrachloride-induced liver fibrosis. Toxicol. Lett. 183(1-3), 36-44. 
1. The Role of Munc13 Isoforms in Regulating Large Dense Core Vesicle Exocytosis in Chromaffin Cells. Core-to-Core Symposium Mechanisms of Synaptic Transmission, Doshisha University, Kyoto, Japan, 2013.

\section{Poster presentations}

1. Man, K.N.M., Pinheiro, P.S., Rhee, J.S., Sørensen, J.B., Brose, N., Wocjik, S.M. The Role of Munc13 Isoforms in Regulating Large Dense Core Vesicle Exocytosis in Chromaffin Cells. ESF-FENS Conference The Neurobiology of Synapses and their Dysfunction, Stresa, Italy, 2013.

2. Man, K.N.M., Yu, M.Y., Cheung, T.H., Yim, S.F., Siu, N.S.S., Chung, T.K.H., Wong, Y.F. MicroRNA signature in cervical neoplasms. Third Biennial Conference of the Asia and Oceania research organization on Genital Infections and Neoplasia (AOGIN), Seoul 2008.

\section{Awards and Stipends}

01/2014-04/2014 PhD Stipend, Max Planck Society

07/2013-12/2013 GGNB Bridging Fund, Georg-August-Universität Göttingen

04/2009-06/2011 Deutscher Akademischer Austausch Dienst (DAAD) Research grant for doctoral candidates and young scientists and academics

05/2008 Prof. Harald zur Hausen Award for Best Proffered Poster, Poster entitled MicroRNA signature in cervical neoplasms,

Third Biennial Conference of the Asia and Oceania research organization on Genital Infections \& Neoplasia (AOGIN), Seoul 2008.

09/2004-08/2006 Postgraduate studentship, University of Hong Kong

2004

Best Presenter, $2^{\text {nd }}$ Departmental Seminar 2004-2005, Department of Zoology, HKU

\section{Techniques Employed in Research Studies}

\section{Electrophysiology}

Whole-cell capacitance measurement, real-time monitoring and control of intracellular calcium concentration, flash photolysis of caged-Ca ${ }^{2+}$ in neurons and neuroendocrine cells.

\section{Cell biology and imaging}

Ex vivo adrenal slice preparation, cryosectioning, immunolabeling, light and fluorescence microscopy, microtome sectioning, staining techniques, tissue culture techniques, transfection/nucleofection, flow cytometry, thymidine incorporation.

\section{Molecular biology}

Semliki Forest Virus preparation for protein expression in neurons and neuroendocrine cells, DNA, RNA and protein extraction from small sample sizes, molecular cloning, DNA sequencing, protein expression and purification in bacterial system, Western blotting, reverse transcription, real-time polymerase chain reaction, laser capture microdissection.

\section{Data analysis}

IgorPro (for electrophysiological data), dChip (for gene expression profile analysis), FlowJo (for flow cytometry data), ImageJ.

\section{Language Ability}

Fluent English, Mandarin and Cantonese

IELTS score (2004): 8.0/9.0

TOEFL score (internet-based test, 2007): 114/120 


\section{$\underline{\text { Referees }}$}

1. Prof. Dr. rer. nat. Nils Brose (Supervisor of doctoral studies)

Head of the Department of the Molecular Neurobiology,

Max Planck Institute of Experimental Medicine, Göttingen, Germany.

Address: Hermann-Rein-Strasse 3

37075 Göttingen

Germany.

Email: $\quad$ brose@em.mpg.de

Phone: $\quad+49(0)$ 5513899-725

Fax: $\quad+49(0) 5513899-715$

2. Dr. Sonja M. Wojcik (Supervisor of doctoral studies)

Group leader at the Department of Molecular Neurobiology,

Max Planck Institute of Experimental Medicine, Göttingen, Germany.

Address: Hermann-Rein-Strasse 3

37075 Göttingen

Germany.

Email: $\quad$ wojcik@em.mpg.de

Phone: $\quad+49$ (0) 5513899-722

3. Prof. Jakob B. Sørensen (Collaborator)

Department of Neuroscience and Pharmacology, University of Copenhagen.

Address: University of Copenhagen - INF

Panum 24-3

Blegdamsvej 3

2200 Copenhagen

Email: $\quad$ jakobbs@sund.ku.dk

Phone: $\quad+45-3532-7931$ 